https://doi.org/10.11646/megataxa.6.1.2

http://zoobank.org/urn:lsid:zoobank.org:pub:E4423D96-48BB-4928-BF54-AD3805321637

\title{
All genera of the world: Order Thysanoptera (Animalia: Arthropoda: Insecta)
}

\author{
LAURENCE A. MOUND ${ }^{1} \&$ ANDREA HASTENPFLUG-VESMANIS ${ }^{2}$ \\ ${ }^{1}$ CSIRO Australian National Insect Collection, PO Box 1700, Canberra, ACT 2601 \\ ="laurence.mound@csiro.au, @ ittps://orcid.org/0000-0002-6019-4762 \\ ${ }^{2}$ Senckenberg Research Institute and Natural History Museum, Senckenberganlage 25, 60325 Frankfurt am Main, Germany \\ "= andrea.hastenpflug-vesmanis@senckenberg.de, @ https://orcid.org/0000-0002-0978-1160
}

\begin{abstract}
There are 1270 genus-group names available in the Insect Order Thysanoptera by the end of 2020. Of these, 850 refer to currently accepted genera, including 65 genera of fossils. A further 420 genus-group names are placed in synonymy. Of the currently accepted genera 474 have been described since 1950. Despite this descriptive activity, the suprageneric classification of Thysanoptera genera remains poorly structured with relationships largely obscure and $50 \%$ of the genera monobasic.
\end{abstract}

Key words: Thysanoptera, generic names, synonyms, type species, thrips

\section{Introduction}

Within the Insecta super-order Thripida two Orders are currently recognised. The smaller Order, Lophioneurida, is known only from fossils and comprises 18 genera (Ulitzka, 2021), whereas the larger Order, Thysanoptera, includes all of the present-day thrips together with a few fossil taxa. The only complete list of genus-group names of Thysanoptera published previously (Priesner 1949b) included a total of 658 names, comprising 517 accepted genera plus 141 synonyms. Partial lists of names in this Order are also available in Bhatti (1993) and a series of seven citation-indices including Jacot-Guillarmod (1974, 1975). The list presented here is almost double in size to that of Priesner, with 1270 genus- group names comprising 850 currently accepted genera, of which 65 refer only to fossils, plus 420 names in synonymy. The increase in the proportion of recognised generic synonyms since 1950 , from $21 \%$ to $33 \%$, represents an increasing emphasis on the systematic relationships between taxa. This contrasts with a traditional emphasis on remarkable structural differences that has resulted in a higher rate of genus-level synonyms among names proposed by authors in the first half of the $20^{\text {th }}$ century (Table 1 ). The increase in number of generic names, an additional 474 recognised genera since 1950, reflects the more than doubling of recognised species, from less than 3000 in 1950 to over 6000 in 2020 (Fig. 1). This has resulted from increased studies on the fauna of tropical areas (Figs 2, 3), including Brazil (Hood; Moulton 1930-1960), Meso-America (Johansen; Retana 1974-2015), Africa (Bournier; zur Strassen 1959-2010), India (Ananthakrishnan; Bhatti 1956-2008), Japan (Okajima; Masumoto 1975-present), and Australia (Mound 1967-present). Despite all this descriptive taxonomy the systematic relationships among the genera of Thysanoptera remain less than satisfactory,

TABLE 1. The number of genus-group names by the most prolific authors.

\begin{tabular}{lccc}
\hline Author & Dates & Currently valid & Currently in synonymy \\
\hline Priesner & $(1891-1974)$ & 104 & 68 \\
Mound & $(1934-)$ & 102 & 5 \\
Hood & $(1899-1966)$ & 97 & 39 \\
Bagnall & $(1889-1962)$ & 61 & 37 \\
Bhatti & $(1939-)$ & 61 & 36 \\
Ananthakrishnan & $(1925-2015)$ & 46 & 29 \\
Moulton & $(1878-1951)$ & 38 & 20 \\
Karny & $(1886-1936)$ & 34 & 28 \\
Okajima & $(1950-)$ & 23 & 2 \\
zur Strassen & $(1926-2013)$ & 23 & 5 \\
\hline
\end{tabular}




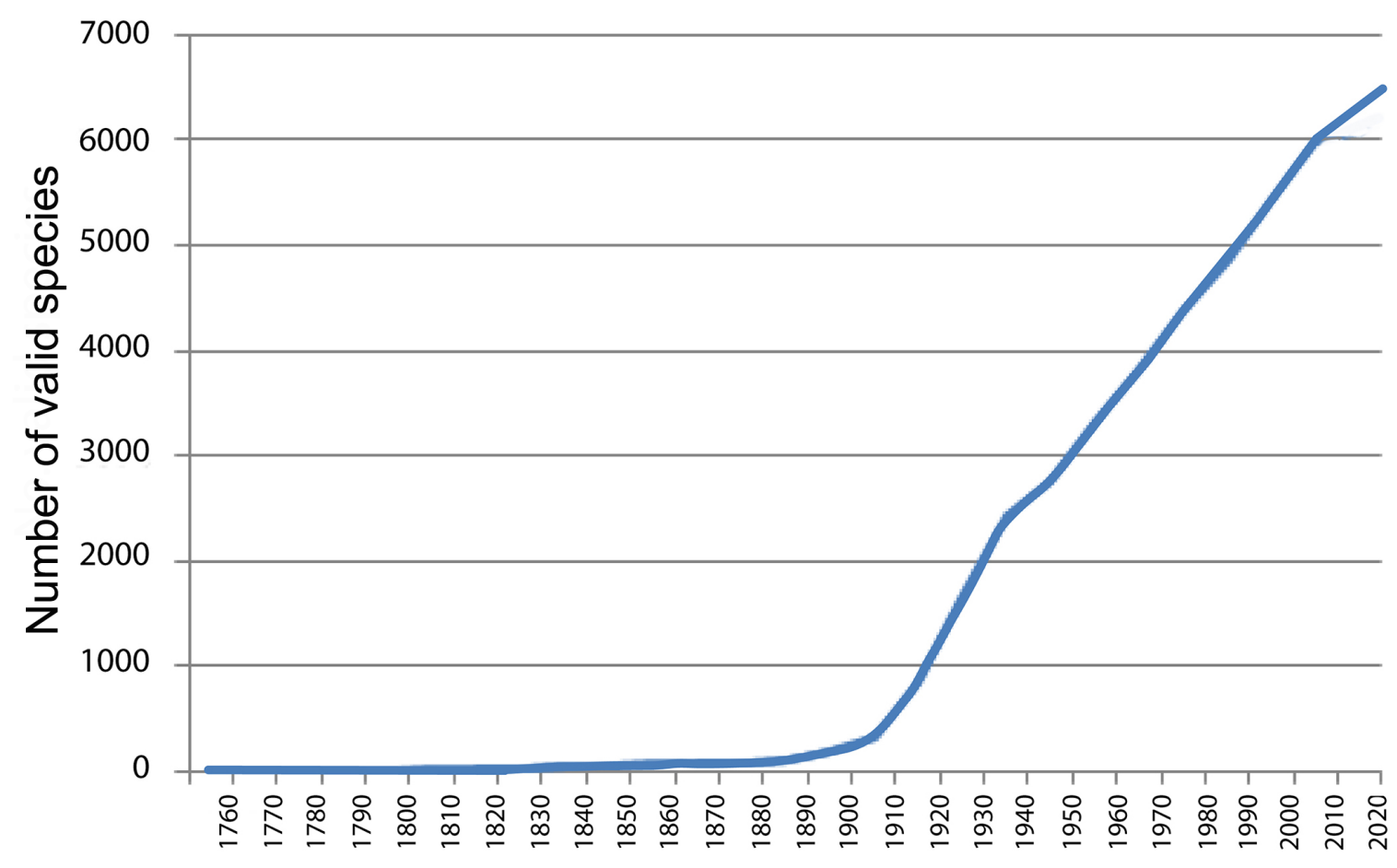

Decade

FIGURE 1. Number of valid Thysanoptera species worldwide by decade.

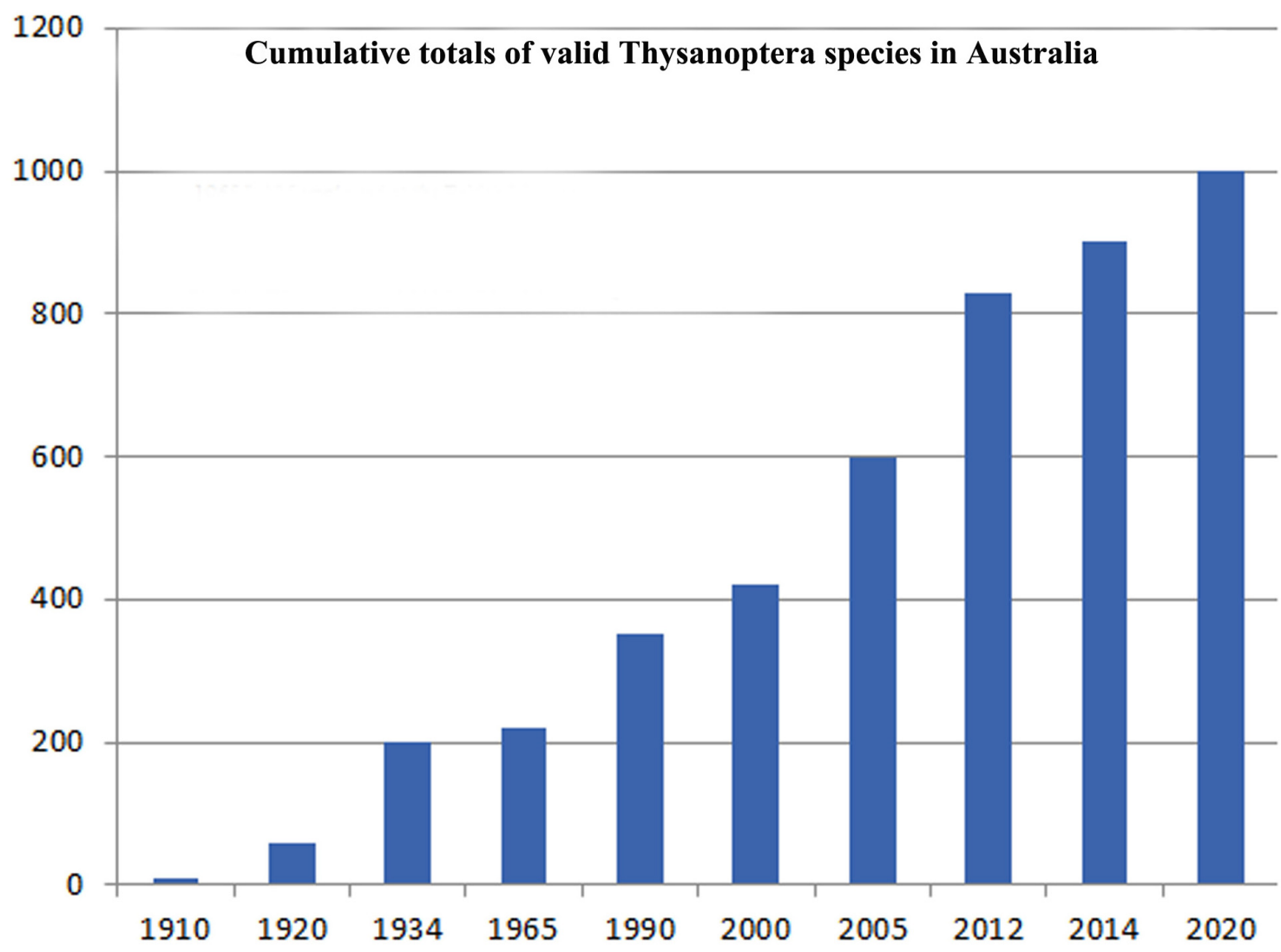

FIGURE 2. Cumulative total of valid Thysanoptera species in Australia. 


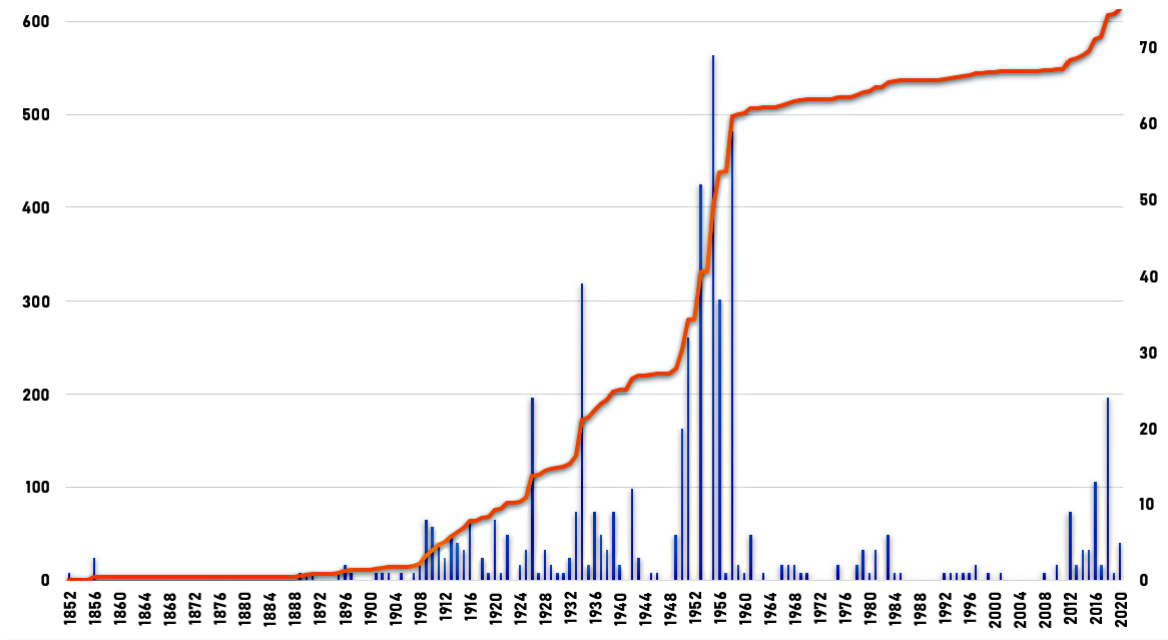

FIGURE 3. Annual and cumulative totals of Thysanoptera species descriptions in Brazil (image provided by Elison Lima).

with 436 of the genera each comprising a single species, a further 230 genera each including no more than five species, and only 10 genera including more than 100 species.

The suprageneric classification within which the genera are listed here is the one available in ThripsWiki (2021) although alternative classifications that have been proposed are indicated and discussed on that web site. Two sub-Orders are recognised, Terebrantia and Tubulifera. In the first of these eight families are recognised that include extant species, together with a further four families that are known only from fossils. In the largest of the Terebrantian families, the Thripidae, four subfamilies are currently recognised. In the second suborder, the Tubulifera, two families are now recognised, of which one comprises a single genus known only from fossils, whereas in the major family, Phlaeothripidae, two subfamilies are recognised. In summary, of the 850 listed genera of Thysanoptera 450 are referred to the Phlaeothripidae and 290 to the Thripidae.

\section{Gender of genus-group names}

The International Code of Zoological Nomenclature (1999) specifies that each species-group name must agree in gender with the genus-group name with which it is combined. In the Insecta Order Thysanoptera all generic names that end with the suffix "thrips" are considered masculine, and the gender of the majority of Thysanoptera genus-group names is thus clear. However, there are 61 Thysanoptera generic names currently in use that do not end with this suffix. Nine of these genusgroup names are considered masculine according to the Code: Carius; Ceratothripoides; Chirothripoides; Corynothripoides; Heliothripoides; Moultonides; Rhinoceps; Rhipidothripoides; Rhopalothripoides. The remaining 52 genus-group names that do not end in "thrips" all end in the letter "a", and since the various authors involved never specified a gender, names such as Ananthakrishnana, Stannardia and Zurstrassenia must be treated as feminine. For many thrips taxonomists this is likely to be counterintuitive, because these names honour male individuals with whom many of us have worked. However, the Code Article 30.2.4 makes the position clear, and thus the gender of all such names is to be considered feminine.

\section{Acknowledgements}

This list of genera is developed from data available on the web in ThripsWiki (2021), a system that was built from information acquired primarily at two museums in Europe. Between 1965 and 1990 a reference library of thrips literature and an associated card catalogue was developed at the Natural History Museum, London. Data from that source has been extensively expanded and corrected through access to the comprehensive literature collection and detailed card catalogues that were produced between 1960 and 2010 at the Senckenberg Museum, Frankfurt, by our late colleague Richard zur Strassen. We are grateful for helpful comments and criticisms by the Megataxa reviewers, particularly Elison Lima and Manfred Ulitzka.

Order Thysanoptera Haliday

(2 suborders; 9 extant families; 6 extinct families)

Sub-Order Terebrantia Haliday

(8 extant families; 5 extinct families)

Family Aeolothripidae Uzel

(24 extant genera; 7 extinct genera) 
Aduncothrips Ananthakrishnan, 1963 [1 species]. Type species Erythrothrips asiaticus Ramakrishna \& Margabandhu, 1931, by monotypy.

Aeolothrips Haliday, 1836 [113 species]. Type species Aeolothrips albicincta Haliday, 1836, by monotypy.

Coleothrips Haliday, 1836. Type species Thrips fasciatus Linneaus, 1758, by subsequent designation of Westwood (1840). Synonymy by Priesner (1925d).

Pygaeolella Priesner, 1926a. Type species Aeolothrips albicincta Haliday, 1836, by subsequent designation of Bagnall (1929g). Synonymy by Priesner (1938c).

Podaeolella Priesner, 1926a. Type species Aeolothrips versicolor Uzel, 1895, by subsequent designation of Bagnall (1929g). Synonymy by Mound et al. (1976).

Orthoaeolothrips Melis, 1932. Nomen nudum, no type species designated.

Plagioaeolothrips Melis, 1932. Nomen nudum, no type species designated.

Aeolothrips (Gevarothrips) Lacasa Plasencia, 1983. Type species Aeolothrips bournieri Lacasa Plasencia, 1983 , by monotypy. Synonymy by zur Strassen (2003).

Fabothrips Bhatti, 1988a. Type species Aeolothrips vittipennis Hood, 1912e, by monotypy. Synonymy by Mound \& Marullo (1996).

Arabthrips Bhatti, 1999b. Type species Aeolothrips asirensis Strassen, 1979, by monotypy. Status uncertain but listed under Aeolothrips in ThripsWiki (2021)

Allelothrips Bagnall, 1932a [7 species]. Type species Allelothrips cincticornis Bagnall, 1932a, by monotypy.

Arhipidothrips Bagnall, 1932a. Type species Arhipidothrips tenuicornis Bagnall, 1932a, by monotypy. Synonymy by Stannard (1961).

Pseudoaeolothrips Bagnall, 1932a. Type species Pseudaeolothrips cameroni Bagnall, 1932a, by monotypy. Synonymy by Stannard (1961).

Ambaeolothrips Mound, Cavalleri, O’Donnell, Infante, Ortiz \& Goldarazena, 2016 [3 species]. Type species Aeolothrips romanruizi Ruiz-De la Cruz et al., 2016, by original designation.

Andrewarthaia Mound, 1967b [1 species]. Type species Rhipidothrips kellyanus Bagnall, 1924b, by original designation.
Audiothrips Moulton, 1930 [2 species]. Type species Audiothrips perplexus Moulton, 1930, by monotypy.

Corynothripoides Bagnall, 1926 [1 species]. Type species Corynothripoides marginipennis Bagnall, 1926, by monotypy.

$\uparrow$ Cretothrips Grimaldi, in Grimaldi, Shmakov \& Fraser 2004 [1 species]. Type species Cretothrips antiquus Grimaldi, 2004, by monotypy.

Cycadothrips Mound, 1991 [3 species]. Type species Cycadothrips chadwicki Mound, 1991, by monotypy.

Dactuliothrips Moulton, 1931 [9 species]. Type species Dactuliothrips spinosus Moulton, 1931, by monotypy.

Desmidothrips Mound, 1977b [3 species]. Type species Desmidothrips walkerae Mound, 1977b, by original designation.

Desmothrips Hood, 1915b [18 species]. Type species Orothrips australis Bagnall, 1914b, by monotypy.

Archaeolothrips Bagnall, 1924e. Type species Archaeolothrips fontis Bagnall, 1924e, by monotypy. Synonym by Bagnall \& Kelly (1928).

Erythridothrips Mound \& Marullo, 1993 [1 species]. Type species Erythridothrips cubilis Mound \& Marullo, 1993, by monotypy.

Erythrothrips Moulton, 1911 [12 species]. Type species Erythrothrips arizonae Moulton, 1911, by monotypy.

Euceratothrips Hood, 1936c [1 species]. Type species Euceratothrips marginipennis Hood, 1936c, by monotypy.

Franklinothrips Back, 1912 [17 species]. Type species Aeolothrips vespiformis Crawford, 1909, by monotypy.

Mitothrips Trybom, 1912a. Type species Mitothrips megalops Trybom, 1912a, by monotypy. Synonymy by Bagnall (1913a).

Spathiothrips Richter, 1928. Type species Spathiothrips bischoffi Richter, 1928, by monotypy. Synonymy by Bagnall (1931).

$\dagger$ Fusithrips Shmakov, 2009 [1 species]. Type species Fusithrips crassipes Shmakov, 2009, by monotypy.

Gelothrips Bhatti, 1967a [3 species]. Type species Aeolothrips (Gelothrips) alis Bhatti, 1967a (=Rhipidothrips cinctus Hood), by monotypy. 
Arcuthrips Mound, 1967b. Type species Desmothrips monrosi de Santis, 1959b, by original designation. Synonymy by Mound (1972a).

Indothrips Bhatti, 1967a [1 1 species]. Type species Indothrips bhushani Bhatti, 1967a, by monotypy.

Lamprothrips Moulton, 1935a [1 species]. Type species Lamprothrips maculosus Moulton, 1935a, by monotypy.

$\dagger$ Lithadothrips Scudder, 1875 [2 species]. Type species Lithadothrips vetustus Scudder, 1875, by monotypy.

Mymarothrips Bagnall, 1928a [4 species]. Type species Mymarothrips ritchianus Bagnall, 1928a, by monotypy.

Orothrips Moulton, 1907 [3 species]. Type species Orothrips kelloggi Moulton, 1907, by monotypy.

Ekplectrothrips Titschack, 1958. Type species Ekplectrothrips priesneri Titschack, 1958, by monotypy. Synonymy by zur Strassen (1989).

$\dagger$ Palaeothrips Scudder, 1875b [1 species]. Type species Palaeothrips fossilis Scudder, 1875, by monotypy.

$\dagger$ Permothrips Martynov, 1935 [1 species]. Type species Permothrips longipennis Martynov, 1935, by monotypy.

Rhipidothripiella Bagnall, 1932a [1 1 species]. Type species Rhipidothrips turneri Moulton, 1930a, by monotypy.

$†$ Rhipidothripoides Bagnall, 1923a [3 species]. Type species Rhipidothripoides abdominalis Bagnall, 1923a, by monotypy.

Rhipidothrips Uzel, 1895 [6 5 species]. Type species Rhipidothrips gratiosus Uzel, 1895, by monotypy.

$\dagger$ Sinaeolothrips Shmakov, 2014 [1 species]. Type species Aeolothrips brodiei Cockerell, 1917, by monotypy.

Stomatothrips Hood, 1911 [8 species]. Type species Stomatothrips flavus Hood, 1911, by monotypy.

Streothrips Bhatti, 1971a [4 species]. Type species Streothrips arorai Bhatti, 1971a, by monotypy.

\section{Family Fauriellidae Priesner (4 extant genera)}

Fauriella Hood, 1937a [1 species]. Type species Fauriella natalensis Hood, 1937a, by monotypy.
Opisthothrips Hood, 1937a [1 species]. Type species Opisthothrips elytropappi Hood, 1937a, by monotypy.

Parrellathrips Mound \& Marullo, 1999 [1 species]. Type species Parrellathrips ullmanae Mound \& Marullo, 1999, by monotypy.

Ropotamothrips Pelikán, 1958 [2 species]. Type species Ropotamothrips buresi Pelikán, 1958, by monotypy.

Osmanothrips Priesner, 1961. Type species Osmanothrips ressli Priesner, 1961, by monotypy. Synonymy by Mound et al. (1980).

\section{Family $\uparrow$ Hemithripidae Bagnall (1 extinct genus)}

$\dagger$ Hemithrips Bagnall, 1923a [9 species]. Type species Hemithrips femoralis Bagnall, 1923a, by original designation.

Family Heterothripidae Bagnall

(4 extant genera; 3 extinct genera)

Aulacothrips Hood, 1952 [5 species]. Type species Aulacothrips dictyotus Hood, 1952, by monotypy.

$\dagger$ Electrothrips Bagnall, 1924d [1 species]. Type species Electrothrips hystrix Bagnall, 1924d, by monotypy.

†Eocephalothrips Bagnall, 1924c [1 1 species]. Type species Thrips capito Schlechtendal, 1887, by monotypy.

Heterothrips Hood, 1908a [76 species]. Type species Heterothrips arisaemae Hood, 1908a, by monotypy.

Lenkothrips de Santis \& Sureda, 1970 [5 species]. Type species Heterothrips (Lenkothrips) sensitivus de Santis \& Sureda, 1970, by monotypy.

$\dagger$ Protothrips Priesner, 1924b [1 species]. Type species Protothrips speratus Priesner, 1924b, by monotypy.

Scutothrips Stannard, 1972 [4 species]. Type species Scutothrips incaensis Stannard, 1972, by original designation.

\section{Family $\uparrow$ Karataothripidae Sharov}

(1 extinct genus)

$\dagger$ Karataothrips Sharov, 1972 [1 species]. Type species Karataothrips jurassicus Sharov, 1972, by monotypy. 
Family $\uparrow$ Liassothripidae Priesner

(1 extinct genus)

$\dagger$ Liassothrips Priesner, 1949b [1 species Mesothrips crassipes Martynov, 1949, by monotypy.

Family Melanthripidae Bagnall

(4 extant genera; 4 extinct genera)

Ankothrips, Crawford, 1909 [15 species]. Type species Ankothrips robustus Crawford, 1909, by monotypy.

Dicranothrips Trybom, 1910. Type species Dicranothrips fissidens Trybom, 1910, by monotypy. Synonymy by Priesner, 1925d.

Prionothrips Schille, 1911. Type species Prionothrips niezabitowskii Schille, 1911, by monotypy. Synonymy by Priesner, 1925 d.

$\dagger$ Archankothrips Priesner, 1924b [5 species]. Type species Archankothrips pugionifer Priesner, 1924b, by monotypy.

Cranothrips Bagnall, 1915a [12 species]. Type species Cranothrips poultoni Bagnall, 1915a, by monotypy.

Dorythrips Hood, 1931a [6 species]. Type species Dorythrips chilensis Hood, 1931a, by monotypy.

$\dagger$ Eocranothrips Bagnall, 1926b [2 species]. Type species Melanothrips annulicornis Bagnall, 1923a, by monotypy.

$\dagger$ Gymnopollisthrips Peñalver, Nel \& Nel, 2012 [2 species]. Type species Gymnopollisthrips minor Peñalver, Nel \& Nel, 2012, by original designation.

Melanthrips Haliday, 1836 [36 species]. Type species Melanthrips obesa Haliday, 1836 (=M. fuscus Sulzer, 1776), by monotypy.

Orthomelanthrips Melis, 1931. Nomen nudum, no type species designated.

Plagiomelanthrips Melis, 1931. Nomen nudum, no type species designated.

Melanthrips (Dichropterothrips) Priesner, 1936b. Type species Melanthrips ficalbii Buffa, 1907.

Turkmenothrips Liskiewicz, 1961. Type species Melanthrips (Turkmenothrips) helenae Liskiewicz, 1961, by monotypy. Synonymy by zur Strassen (1980a). $\dagger$ Proboscisthrips Ulitzka, 2017 [1 species]. Type species Proboscisthrips mammuthoides Ulitzka, 2017, by original designation.

Family Merothripidae Bagnall

( 3 extant genera; 3 extinct genera)

Damerothrips Hood, 1954a [1 species]. Type species Damerothrips gemmatus Hood, 1954a, by monotypy.

Erotidothrips Priesner, 1939c [1 species]. Type species Erotidothrips mirabilis Priesner, 1939c, by monotypy.

$\dagger$ †ezzinothrips zur Strassen, 1973 [1 1 species]. Type species Jezzinothrips cretacicus zur Strassen, 1973, by monotypy.

Merothrips Hood, 1912e [19 species]. Type species Merothrips morgani Hood, 1912e, by monotypy.

Amerothrips Bhatti, 1989. Type species Merothrips fusciceps Hood \& Williams, 1915, by monotypy. Synonymy by Mound \& Marullo (1996).

Sakimurathrips Bhatti, 1989. Type species Merothrips williamsi Priesner, 1921c, by monotypy. Synonymy by Mound \& Marullo (1996).

Tardothrips Bhatti, 1989. Type species Merothrips mirus Crawford, 1942, by monotypy. Synonymy by Mound \& Marullo (1996).

$\dagger$ Myanmarothrips Ulitzka, 2018 [1 species]. Type species Myanmarothrips pankowskiorum Ulitzka, 2018, by monotypy

$\dagger$ Praemerothrips Priesner, 1929a [1 species]. Type species Praemerothrips hoodi Priesner, 1929a, by monotypy.

Family Stenurothripidae Bagnall

(3 extant genera; 10 extinct genera)

$†$ Cenomanithrips Tong, Shih \& Ren, 2019 [1 species]. Type species Cenomanithrips primus Tong, Shih \& Ren, 2019, by monotypy.

$\dagger$ Exitelothrips zur Strassen, 1973 [1 1 species]. Type species Exitelothrips mesozoicus zur Strassen, 1973, by monotypy.

Heratythrips Mound \& Marullo, 1999 [1 species]. Type species Heratythrips sauli Mound \& Marullo, 1999, by monotypy. 
$\dagger$ Hispanothrips Peñalver \& Nel, 2010 [1 species]. Type species Hispanothrips utrillensis Peñalver \& Nel, 2010, by monotypy.

Holarthrothrips Bagnall, 1927b [6 species]. Type species Holarthrothrips tenuicornis Bagnall, 1927b, by monotypy.

Adiheterothrips Ramakrishna, 1928. Type species Adiheterothrips jambudvipae Ramakrishna, 1928, by monotypy. Synonymy by Mound et al. (1980).

$\uparrow$ Neocomothrips zur Strassen, 1973 [1 species]. Type species Neocomothrips hennigianus zur Strassen, 1973 , by monotypy.

Oligothrips Moulton, 1933c [1 species]. Type species Oligothrips oreius Moulton, 1933c, by monotypy.

†Opadothrips Priesner, 1924b [2 species]. Type species Opadothrips fritschianus Priesner, 1924b, by monotypy.

$†$ Progonothrips zur Strassen, 1973 [1 species]. Type species Progonothrips horridus zur Strassen, 1973, by monotypy.

$\dagger$ Rhetinothrips zur Strassen, 1973 [1 species Rhetinothrips elegans zur Strassen, 1973, by monotypy.

$\dagger$ Scaphothrips zur Strassen, 1973 [1 1 species]. Type species Scaphothrips antennatus zur Strassen, 1973, by monotypy.

$†$ Scudderothrips zur Strassen, 1973 [1 1 species]. Type species Scudderothrips sucinus zur Strassen, 1973, by monotypy.

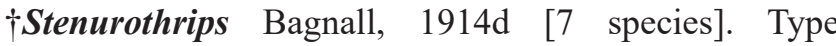
species Stenurothrips succineus Bagnall, 1914d, by monotypy.

\section{Family Thripidae Stephens}

(4 extant sub-families)

Sub-family Dendrothripinae Priesner

(12 extant genera; 4 extinct genera)

$\dagger$ Apodendrothrips Schliephake, 1993 [1 species]. Type species Apodendrothrips major Schliephake, 1993, by monotypy.

Asprothrips Crawford, 1938 [8 species]. Type species Asprothrips raui Crawford, 1938 (=Euthrips seminigricornis Girault, 1926), by monotypy.
Dendrothrips Uzel, 1895 [56 species]. Type species Dendrothrips tiliae Uzel, 1895 (=Thrips ornatus Jablonowski, 1894), by subsequent designation of Priesner (1921a)

Monochaetella Priesner, 1921a. Type species Dendrothrips saltatrix Uzel, 1895, by monotypy. Synonymy by zur Strassen (1968b).

Dichaetella Priesner, 1921a. Type species Dendrothrips (Dichaetella) karnyi Priesner, 1921a, by monotypy. Synonymy by zur Strassen (1968b).

Dendrothripiella Bagnall, 1927a. Type species Dendrothrips phyllireae Bagnall, 1927a, by monotypy. Synonymy by zur Strassen (1968b).

Dendrothrips (Vicinichaetella) Yakhontov, 1958. Type species Dendrothrips (Vicinichaetella) saniishi Yakhontov, 1958, by monotypy. Synonymy by zur Strassen (1968b).

Cerothrips Ananthakrishnan, 1961b. Type species Cerothrips minutus Ananthakrishnan, 1961b, by monotypy. Synonymy by zur Strassen (1968b).

Edissa Faure, 1953 [2 species]. Type species Edissa flava Faure, 1953, by monotypy.

Ensiferothrips Bianchi, 1945 [4 species]. Type species Ensiferothrips primus Bianchi, 1945, by monotypy.

Filicopsothrips Mound, 1999 [2 species]. Type species Filicopsothrips wellsae Mound, 1999, by monotypy.

Halmathrips Hood, 1936b [5 species]. Type species Halmathrips citricinctus Hood, 1936b, by monotypy.

Halmathrips (Phaosothrips) Stannard, $1953 b$. Type species Halmathrips (Phaosothrips) beckeri Stannard, 1953b, by monotypy. Synonymy by Mound \& Marullo (1996).

Halmathrips (Demetriothrips) Johansen, 1974. Type species Halmathrips (Demetriothrips) ferruginus Johansen, 1974, by monotypy. Synonymy by Mound \& Marullo (1996).

Iranodendrothrips Alavi, Minaei \& Fekrat 2014 [1 species]. Type species Iranodendrothrips kamalii Alavi, Minaei \& Fekrat, 2014, by monotypy.

Leucothrips Reuter, 1904 [5 species]. Type species Leucothrips nigripennis Reuter, 1904, by monotypy.

Microthrips Morgan, 1913. Type species Microthrips piercei Morgan, 1913, by monotypy. Synonymy by Hood (1931b). 
Sporangiothrips Daniel, 1985. Type species Sporangiothrips acuminatus Daniel, 1985, by monotypy. Synonymy by Bhatti (1998a).

Parsiothrips Bhatti, 1970b [1 1 species]. Type species Parsiothrips fuscus Bhatti, 1970b, by monotypy.

Petrothrips Masumoto \& Okajima, 2003 [1 species]. Type species Petrothrips nigriceps Masumoto \& Okajima, 2003, by monotypy.

$\dagger$ Praedendrothrips Priesner, 1924b [1 species]. Type species Praedendrothrips avus Priesner, 1924b, by monotypy.

Projectothripoides Shumser Singh, 1942 [1 species]. Type species Projectothripoides pandai Shumser Singh, 1942, by monotypy.

Pseudodendrothrips Schmutz, 1913 [21 species]. Type species Pseudodendrothrips ornatissimus Schmutz, 1913, by monotypy.

Graphidothrips Moulton, 1930b. Type species Graphidothrips stuardoi Moulton, 1930b, by monotypy. Synonymy by Bhatti (1969a).

Phlebothrips Priesner, 1965. Type species Phlebothrips aegyptiacus Priesner, 1965, by monotypy. Synonymy by zur Strassen (1993).

$†$ Schedodendrothrips Schliephake, 2001b [1 species]. Type species Schedodendrothrips ursulae Schliephake, 2001b, by monotypy.

$†$ Synnastothrips Schliephake, 1993 [2 species]. Type species Synnastothrips vitreus Schliephake, 1993, by original designation.

\section{Sub-family Panchaetothripinae Bagnall \\ (41 extant genera; 3 extinct genera)}

Anisopilothrips Stannard \& Mitri, 1962 [1 species]. Type species Heliothrips venustulus Priesner, 1923, by monotypy.

Aoratothrips Priesner, 1933c [1 species]. Type species Aoratothrips tenuis Priesner, 1933c, by monotypy.

Arachisothrips Stannard, 1952b [3 species]. Type species Arachisothrips millsi Stannard, 1952b, by original designation.

Araliacothrips Li, Li \& Zhang, 2018 [1 species]. Type species Araliacothrips daweishanensis Li, Li \& Zhang, 2018, by monotypy.

$†$ Archaeothrips Priesner, 1924b [1 1 species]. Type species Archaeothrips latipennis Priesner, 1924b, by monotypy.
Gerontothrips Priesner, 1949b. Unnecessary replacement name (Bhatti, 2006).

Astrothrips Karny, 1921a [13 species]. Type species Heliothrips globiceps Karny, 1913a, by subsequent designation of Moulton (1932).

Astrothrips (Gamothrips) Priesner, 1965. Type species Astrothrips (Gamothrips) connaticornis Priesner,1965, by monotypy. Synonymy by Mound (1968a).

Sempothrips Bhatti, 1967a. Type species Sempothrips asiaticus Bhatti, 1967a, by monotypy. Synonymy by Wilson (1975).

Brevithrips Bhatti, 1967a. Type species Astrothrips tumiceps Karny, 1923, by monotypy. Synonymy by Wilson (1975).

Astrothrips (Proxothrips) Bhatti, 1967a. Type species Astrothrips (Proxythrips) lantana Bhatti, 1967a, by monotypy. Synonymy by Wilson (1975).

Australothrips Bagnall, 1916a [2 species]. Type species Australothrips bicolor Bagnall, 1916a, by monotypy.

Pterothrips Hood, 1918. Type species Pterothrips quadratus Hood, 1918, by monotypy. Synonymy by Bagnall (1932b).

Bhattithrips Mound, 1970b [4 species]. Type species Heliothrips frontalis Bagnall, 1916a, by original designation.

Brachyurothrips Bagnall, 1921a [1 1 species]. Type species Brachyurothrips anomalus Bagnall, 1921a, by monotypy.

Bradinothrips Hood, 1954g [2 species]. Type species Bradinothrips williamsi Hood, 1954g, by monotypy.

Palleucothrips Hood, 1956b. Type species Palleucothrips musae Hood, 1956b, by monotypy. Synonymy by Mound \& Marullo (1996).

Caliothrips Daniel, 1904 [25 species]. Type species Caliothrips woodworthi Daniel, 1904, by monotypy.

Hercothrips Hood, 1928. Type species Heliothrips striatus Hood, 1913c, by original designation. Synonymy by Wilson (1975).

Chaeturothrips Hood, 1954f [1 species]. Type species Chaeturothrips machadoi Hood, 1954f, by monotypy.

†Coccothrips Shmakov, 2014 [1 species]. Type species Coccothrips hoffeinsorum Shmakov, 2014, by monotypy. 
Copidothrips Hood, 1954e [1 species]. Type species Copidothrips formosus Hood, 1954e (=Heliothrips (Parthenothrips) octarticulata Schmutz, 1913), by monotypy.

Mesostenothrips Stannard \& Mitri, 1962. Type species Mesostenothrips kraussi Stannard \& Mitri, 1962, by monotypy. Synonymy by Bhatti (1967a).

Dinurothrips Hood, 1913b [2 species]. Type species Dinurothrips hookeri Hood, 1913b, by monotypy.

Elixothrips Stannard \& Mitri, 1962 [1 species]. Type species Tryphactothrips brevisetis Bagnall, 1921a, by monotypy.

Hoodothripiella Retana-Salazar, 2007a. Type species Hoodothripiella ignacio Retana-Salazar, 2007a, by monotypy. Synonymy by Lima et al. (2020).

Euhydatothrips Bagnall, 1926c [2 species]. Type species Euhydatothrips nigripennis Bagnall, 1926c, by monotypy.

Euidothrips Ananthakrishnan, 1968a [4 species]. Type species Euidothrips apsarus Ananthakrishnan, 1968 a, by monotypy.

Galeothrips Nonaka \& Okajima, 1992 [1 species]. Type species Galeothrips seticornis Nonaka \& Okajima, 1992, by monotypy.

Helionothrips Bagnall, 1932b [29 species]. Type species Heliothrips brunneipennis Bagnall, 1915a, by monotypy.

Charientothrips Ananthakrishnan, 1967. Type species Charientothrips nilgiricus Ananthakrishnan,1967, by monotypy. Synonymy by Wilson (1975).

Heliothrips Haliday, 1836 [6 species]. Type species Heliothrips adonidum Haliday, 1836 (=Thrips haemorrhoidalis Bouché,1833), by monotypy.

Hemingia Reyes, 1994 [1 species]. Type species Hemingia glandula Reyes, 1994, by monotypy.

Hercinothrips Bagnall, 1932b [10 species]. Type species Heliothrips bicinctus Bagnall, 1919, by original designation.

$\dagger$ Hoffeinsithrips Schliephake, 2001a [1 species]. Type species Hoffeinsithrips teuberi Schliephake, 2001a, by monotypy.

Hoodothrips Bondar, 1931 [3 species]. Type species Hoodothrips neivai Bondar, 1931, by monotypy.
Hoodothripoides Mound, 1970b. Type species Hoodothripoides braziliensis Mound, 1970b, by monotypy. Synonymy by Mound \& Marullo (1996).

Monilothrips Moulton, 1929a [1 species]. Type species Monilothrips kempi Moulton,1929a, by monotypy.

Moundothrips Wilson, 1975 [1 species]. Type species Moundothrips apterygus Wilson, 1975, by monotypy.

Neoheliothrips Nakahara, O’Donnell \& Mound, 2015 [1 species]. Type species Heliothrips sylvanus Faure, 1933, by monotypy.

Noathrips Bhatti, 1967a [1 1 species]. Type species Noathrips prakashi Bhatti, 1967a, by monotypy.

Oneilliella Wilson, 1975 [1 species]. Type species Hercothrips williamsi Hood, 1928, by monotypy.

Opimothrips Nonaka \& Okajima, 1992 [1 species]. Type species Opimothrips tubulatus Nonaka \& Okajima, 1992, by monotypy.

Panchaetothrips Bagnall, 1912 [7 species]. Type species Panchaetothrips indicus Bagnall, 1912, by monotypy.

Parascolothrips Mound, 1967a [1 species]. Type species Parascolothrips priesneri Mound, 1967a, by monotypy.

Parthenothrips Uzel, 1895 [1 species]. Type species Heliothrips dracaenae Heeger, 1854, by monotypy.

Phibalothrips Hood, 1918 [4 species]. Type species Phibalothrips exilis Hood, 1918 (=Heliothrips longiceps Karny, 1913a), by monotypy.

Reticulothrips Faure, 1925. Type species Reticulothrips peringueyi Faure, by monotypy. Synonymy by Jacot-Guillarmod (1937).

Retithrips Marchal, 1910 [2 species]. Type species Retithrips aegyptiacus Marchal, 1910 (=Thrips (Heliothrips) syriacus Mayet, 1890), by monotypy.

Stylothrips Bondar, 1924. Type species Stylothrips bondari Bondar, 1924, by monotypy. Synonymy by Priesner (1949b).

Rhipiphorothrips Morgan, 1913 [5 species]. Type species Rhipiphorothrips pulchellus Morgan, 1913, by monotypy.

Selenothrips Karny, 1911a [1 species]. Type species Physopus rubrocincta Giard, 1901, by monotypy. 
Sigmothrips Ward, 1970 [1 species]. Type species Sigmothrips aotearoana Ward, 1970, by monotypy.

Stannardiola Wilson, 1975 [3 species]. Type species Stannardiola capitalis Wilson, 1975, by original designation.

Stosicthrips Mound, 2009 [1 1 species]. Type species Stosicthrips szitas Mound, 2009, by monotypy.

Tryphactothrips Bagnall, 1919 [1 species]. Type species Dinurothrips rutherfordi Bagnall, 1915a, by original designation.

Xestothrips Priesner, 1938b [1 species]. Type species Selenothrips (Xestothrips) glabratus Priesner, 1938b, by monotypy.

Zaniothrips Bhatti, 1967a [1 species]. Type species Zaniothrips ricini Bhatti, 1967a, by monotypy.

\section{Sub-family Sericothripinae Karny \\ (3 extant genera; 0 extinct genera)}

Hydatothrips Karny, 1913d [44 species]. Type species Hydatothrips adolfifriderici Karny, 1913d, by monotypy.

Zonothrips Priesner, 1926c. Type species Zonothrips karnyi Priesner, 1926c, by monotypy. Synonymy by Wang (2007).

Corcithrips Bhatti, 1973a. Type species Corcithrips hartwigi Bhatti,1973a, by monotypy. Synonymy by Wang (2007).

Hydatothrips (Pyrothrips) Bhatti, 1973a. Type species Sericothrips (Hydatothrips) boerhaaviae Seshadri \& Ananthakrishnan, 1954, by monotypy. Synonymy by Wang (2007).

Neohydatothrips John, 1929 [121 species]. Type species Neohydatothrips latereostriatus John, 1929 (=Sericothrips portoricensis Morgan, 1925), by monotypy.

Elbuthrips Bhatti, 1973a. Type species Elbuthrips latis Bhatti, 1973a, by monotypy. Synonymy by Wang (2007).

Faureana Bhatti, 1973a. Type species Zonothrips smutsi Faure, 1957, by monotypy. Synonymy by Mound \& Tree (2009).

Kazinothrips Bhatti, 1973a. Type species Zonothrips luridus Ananthakrishnan 1968a, by original designation. Synonymy by Wang (2007).
Neohydatothrips (Onihothrips) Bhatti, 1973a. Type species Sericothrips formosus Faure, 1958b, by monotypy. Synonymy by Mound \& Tree (2009).

Sariathrips Bhatti, 1990b. Type species Sericothrips masrensis Priesner, 1965, by monotypy. Synonymy by Mound \& Tree (2009).

Sensothrips Bhatti, 1999d. Type species Hydatothrips (Kazinothrips) reticulatus Kudo, 1991, by monotypy. Synonymy by Wang (2007).

Piliothrips Bhatti, 2006. Type species Sericothrips gracilicornis Williams, 1916, by original designation. Synonymy by Mound \& Tree (2009).

Sericothrips Haliday, 1836 [7 species]. Type species Sericothrips staphylinus Haliday, 1836, by monotypy.

Rhytidothrips Karny, 1910.Type species Rhytidothrips bicornis Karny, 1910, by monotypy. Synonymy by Karny (1913a).

Sussericothrips Han, 1991. Type species Sussericothrips melilotus Han, 1991, by monotypy. Synonymy by Mound \& Tree (2009).

Sub-family Thripinae Stephens (229 extant genera; 13 extinct genera)

Abacothrips Bhatti, 1986 [1 species]. Type species Abacothrips lotus Bhatti, 1986, by monotypy.

Acremonothrips Priesner, 1939c [1 species]. Type species Taeniothrips aethiops Hood, 1925d, by monotypy.

Adelphithrips Mound \& Palmer, 1981 [3 species]. Type species Adelphithrips nothofagi Mound \& Palmer, 1981, by original designation.

Afrothripella Bhatti, 1990a [1 species]. Type species Chirothrips erinaceus zur Strassen, 1969, by monotypy.

Agalmothrips Priesner, 1965 [1 species]. Type species Anaphothrips (Agalmothrips) parviceps Priesner, 1965 , by monotypy.

Agerothrips Trybom, 1910 [1 1 species]. Type species Agerothrips badius Trybom, 1910, by monotypy.

Agriothrips Ananthakrishnan, 1966b [1 species]. Type species Agriothrips brevisetosus Ananthakrishnan, 1966b, by monotypy.

Ajothrips Bhatti, 1967b [3 species]. Type species Ajothrips karma Bhatti, 1967b, by original designation. 
Akheta Bhatti, 1978d [2 species]. Type species Perissothrips (Bdalsidothrips) brevicornis Bournier, 1967 , by monotypy.

Alathrips Bhatti, 1970a [1 1 species]. Type species Hyalopterothrips roonwali Bhatti, 1963, by monotypy.

Aliceathrips Mound, 2011 [5 species]. Type species Aliceathrips mnestes Mound, 2011, by original designation.

Amalothrips Ananthakrishnan, 1967 [2 species]. Type species Amalothrips flaccidus Ananthakrishnan, 1967, by monotypy.

Ameranathrips Mound \& Marullo, 1996 [1 species]. Type species Ameranathrips herediae Mound \& Marullo, 1996, by monotypy.

Amomothrips Bhatti, 1978c [1 species]. Type species Taeniothrips associatus Priesner, 1938d, by monotypy.

$†$ Amorphothrips Bagnall, 1924 [1 species]. Type species Amorphothrips klebsi Bagnall, 1924, by monotypy.

Amphithrips Ananthakrishnan, 1965a [1 species]. Type species Amphithrips argutus Ananthakrishnan, 1965a, by monotypy.

Amphorothrips Priesner, 1939c [1 species]. Type species Amphorothrips reticulatus Priesner, 1939c, by monotypy.

Anaphothrips Uzel, 1895 [86 species]. Type species Anaphothrips virgo Uzel, 1895 (=Thrips obscura Müller, 1776), by subsequent designation of Hood (1914b).

Neophysopus Schmutz, 1913. Type species Neophysopus medioflavus Schmutz, 1913, by monotypy. Synonymy by Bhatti (1978b).

Pseudoarticulella Shumser Singh, 1942. Type species Thrips obscura Müller, 1776, by monotypy. Synonymy by Priesner (1949b).

Othinanaphothrips Crawford, 1943a. Type species Othinanaphothrips spilleri Crawford, 1943a, by monotypy. Synonymy by Pitkin (1978).

Nakaharathrips Retana-Salazar, 2007b. Type species Anaphothrips sudanensis Trybom, 1911, by original designation. Synonymy by Goldarazena et al. (2008).

Anaphrygmothrips Mound \& Walker, 1982 [1 species]. Type species Anaphrygmothrips otagensis Mound \& Walker, 1982, by monotypy.
Anarrhinothrips Pelikán, 1984 [1 species]. Type species Anarrhinothrips mongolicus Pelikán, 1984, by monotypy.

Anascirtothrips Bhatti, 1961 [4 species]. Type species Anascirtothrips arorai Bhatti, 1961, by monotypy.

Aneristothrips De Santis, 1957 [2 species]. Type species Aneristothrips rostratus De Santis, 1957, by monotypy.

Aneurothrips Karny, 1912b [2 species]. Type species Aneurothrips punctipennis Karny, 1912b, by monotypy.

Apsilothrips Bhatti \& de Borbón, 2008 [1 species]. Type species Apsilothrips atriplex Bhatti \& de Borbón, 2008, by monotypy.

Apterothrips Bagnall, 1908c [2 species]. Type species Apterothrips subreticulata Bagnall, 1908c (=Thrips secticornis Trybom, 1896), by monotypy.

Aptinothrips Haliday, 1836 [5 species]. Type species Thrips (Aptinothrips) rufa Haliday, 1836, by subsequent designation of Mound \& Palmer (1974).

Uzeliella Bagnall, 1908a. Type species Uzeliella lubbocki Bagnall, 1908a(=Thrips rufa Haliday, 1836), by monotypy. Synonymy by Priesner (1925d).

Carinopleuris Bagnall, 1908a. Nomen nudum. Type species Uzeliella lubbocki Bagnall, 1908a, by monotypy.

Apithrips Dyadechko, 1964. Type species Aptinothrips rufus $f$. intermedia Priesner, 1920b, by monotypy. Synonymy by Jacot-Guillarmod (1974).

Apothrips Dyadechko, 1964. Erected for two species (Aptinothrips stylifera Trybom, 1894; Aptinothrips karnyi John, 1927) but no type species designated. Synonymy by Jacot-Guillarmod (1974).

Arenathrips Velikan, 1986 [1 species]. Type species Arenathrips apricus Velikan, 1986, by monotypy.

Aroidothrips Ananthakrishnan, 1960 [1 species]. Type species Aroidothrips longistylus Ananthakrishnan, 1960, by monotypy.

Arorathrips Bhatti, 1990a [16 species]. Type species Chirothrips mexicanus Crawford, 1909, by original designation.

Arpediothrips Hood, 1927b [2 species]. Type species Arpediothrips mojave Hood, 1927b, by monotypy. 
Asphodelothrips zur Strassen, 1995 [2 species]. Type species Pezothrips croceicollis Karny, 1914a, by original designation.

Croceithrips Bhatti, 1995a. Type species Taeniothrips croceicollis Costa, 1888, by original designation. Synonymy by Bhatti (1999b).

Aurantothrips Bhatti, 1978b [2 species]. Type species Anaphothrips orchidaceus Bagnall, 1909b, by monotypy.

Nicolemma Retana-Salazar, 2007b. Type species Anaphothrips orchidaceus Bagnall, 1909 b. Synonymy by Goldarazena et al., 2008.

Ayyaria Karny, 1926 [1 species]. Type species Ayyaria chaetophora Karny, 1926, by monotypy.

Bussothrips Moulton, 1935b. Typespecies Bussothrips claratibia Moulton, 1935b, by monotypy. Synonymy by Priesner (1949b).

Bacathrips Bhatti, 1990b[1 species]. Type species Hydatothrips solanifolii Shumser, 1944, by monotypy.

Baileyothrips Kono \& O’Neill, 1964 [2 species]. Type species Anaphothrips arizonensis Morgan, 1913, by monotypy.

Baliothrips Uzel, 1895 [3 species]. Type species Thrips dispar Haliday, 1836, by monotypy.

Euchaetothrips Bagnall, 1916b. Type species Thrips kroli Schille, 1911, by monotypy. Synonymy by Mound (1968a).

$\dagger$ Balticothrips Schliephake, 1999 [1 1 species]. Type species Taeniothrips (Balticothrips) streckelsbergi Schliephake, 1999, by monotypy.

Bathrips Bhatti, 1962 [2 species]. Type species Taeniothrips melanicornis Shumser Singh, 1946, by monotypy.

Belothrips Haliday, 1836 [2 species]. Type species Taeniothrips (Belothrips) acuminatus Haliday, 1836, by monotypy.

Bhattiana Bournier, 1979 [1 species]. Type species Bhattiana angolensis Bournier, 1979, by monotypy.

Biltothrips Bhatti, 1973a [2 species]. Type species Sericothrips minutus Bhatti, 1967a, by monotypy.

Blascothrips zur Strassen, 1997 [1 1 species]. Type species Blascothrips zumetai zur Strassen, 1997, by monotypy.
Bolacothrips Uzel, 1895 [13 species]. Type species Bolacothrips jordani Uzel, 1895, by monotypy.

Bolacidothrips Priesner, 1930b. Type species Bolacidothrips graminis Priesner, 1930b, by monotypy. Synonymy by Bhatti (1983).

Bournierothrips Bhatti, 1979b [5 species]. Type species Plesiopsothrips barrai Bournier, 1970, by original designation.

Bravothrips Johansen, 1986b [4 species]. Type species Bravothrips daturae Johansen, 1986b, by original designation.

Bregmatothrips Hood, 1912b [11 species]. Type species Bregmatothrips venustus Hood, 1912b, by original designation.

Limocercyothrips Watson, 1926. Type species Limocercyothrips bicolor Watson, 1926, by monotypy. Synonymy by Priesner (1949b).

Poethrips Faure, 1933. Type species Poethrips furcatus Faure, 1933, by monotypy. Synonymy by Bhatti (1984).

Neolimothrips Shumsher, 1942. Type species Neolimothrips brachycephalus Shumsher, 1942, by monotypy. Synonymy by Mound (1968a).

Brooksithrips Retana \& Mound, 2005 [1 species]. Type species Brooksithrips chamaedoreae Retana \& Mound, 2005, by monotypy.

†Calothrips Oustalet, 1873 [1 species]. Type species Calothrips scudderi Oustalet, 1873, by monotypy.

Capitothrips Bhatti, 1974 [1 species]. Type species Capitothrips subramanii Bhatti, 1974, by monotypy.

Caprithrips Faure, 1933 [6 5 species]. Type species Caprithrips analis Faure, 1933, by monotypy.

Bandamia zur Strassen, 1965. Type species Aptinothrips melanophthalmus Bagnall, 1927b, by monotypy. Synonymy by Bhatti (1973b).

Catinathrips O’Neill, 1967 [5 species]. Type species Catinathrips kainos O’Neill, 1967, by original designation.

Ceratothripoides Bagnall, 1918 [5 species]. Type species Ceratothripoides brunneus Bagnall, 1918, by monotypy.

Ceratothrips Reuter, 1899 [1 species]. Type species Ceratothrips trybomi Reuter, 1899 (=Thrips ericae Haliday, 1836), by monotypy. 
Amblythrips Bagnall, 1911a. Type species Amblythrips ericae Bagnall, 1911a, by original designation. Synonymy by Jacot-Guillarmod (1974).

Oxythrips (Caenothrips) Hood, 1916d. Type species Thrips ericae Haliday,1836, by subsequent designation of Priesner (1949b). Synonymy of Amblythrips by Priesner (1949b).

Cercyothrips Morgan, 1925 [2 species]. Type species Cercyothrips striatus Morgan, 1925, by monotypy.

Cestrothrips Priesner, 1964 [1 species]. Type species Rhamphothrips karnyi Bagnall, 1927b, by monotypy.

Chaetanaphothrips Priesner, 1925d [20 species]. Type species Euthrips orchidii Moulton, 1907, by monotypy.

Chaetisothrips Priesner, 1957 [7 species]. Type species Isochaetothrips striatus Hood, 1935b, by original designation.

Charassothrips Hood, 1954c [5 species]. Type species Charassothrips urospathae Hood, 1954c, by monotypy.

Humboldthrips Johansen, 1983b. Type species Humboldthrips incomparabilis Johansen, 1983b, by monotypy. Synonymy by Mound \& Marullo (1996).

Chirothrips Haliday, 1836 [42 species]. Type species Thrips (Chirothrips) manicata Haliday, 1836, by monotypy.

Agrostothrips Hood, 1954b. Type species Agrostothrips guillarmodi Hood, 1954b, by monotype. Synonymy by Minaei \& Mound (2010).

Clypeothrips Nonaka \& Jangvitaya, 1993 [2 species]. Type species Clypeothrips tibialis Nonaka et Jangvitaya, 1993, by monotypy.

Collembolothrips Priesner, 1935d [2 species]. Type species Collembolothrips mediterraneus Priesner, 1935 d, by monotypy.

†Convexithrips Shmakov, 2009. [1 1 species]. Type species Convexithrips robustus Shmakov, 2009, by monotypy.

Coremothrips Hood, 1925c [2 species]. Type species Coremothrips pallidus Hood, 1925c, by monotypy.

Corynothrips Williams, 1913 [3 species]. Type species Corynothrips stenopterus Williams, 1913, by monotypy.
Craspedothrips zur Strassen, 1966 [10 species]. Type species Physothrips hargreavesi Karny, 1925a, by monotypy.

Toxothrips Bhatti, 1967a. Type species Toxothrips ricinus Bhatti, 1967a, by monotypy. Synonymy by Bhatti (1978c).

Tsengothrips Chen, 1976. Type species Tsengothrips plumosa Chen, 1976, by monotypy. Synonymy by Bhatti (1990b).

Craspedothrips (Antenothrips) Bhatti, 1995. Type species Physothrips antennatus Bagnall, 1914a, by original designation. Synonymy by Mound et al. (2012).

Cricothrips Trybom, 1913 [4 species]. Type species Cricothrips karnyi Trybom, 1913, by monotypy.

Plesiopsothrips Hood, 1956a. Type species Plesiopsothrips trinidadensis Hood, 1956a, by monotypy. Synonymy by Bhatti (1990b).

Moundiella Bournier \& Bournier, 1988. Type species Moundiella bourbonensis Bournier \& Bournier, 1988 , by original designation. Synonymy by Bhatti (1990b).

Ctenidothrips Priesner, 1952a [1 species]. Type species Ctenidothrips bambusae Priesner, 1952a, by monotypy.

Ctenothrips Franklin, 1907 [5 species]. Type species Ctenothrips bridwelli Franklin, 1907, by monotypy.

Cyrilthrips Tree \& Mound, 2009 [1 1 species]. Type species Cyrilthrips cecidis Tree \& Mound, 2009, by monotypy.

Danothrips Bhatti, 1971b [11 species]. Type species Danothrips setifer Bhatti, 1971b, by monotypy.

Decorothrips Bhatti, 1973b [1 species]. Type species Caprithrips decorus Faure, 1940, by monotypy.

Dendrothripoides Bagnall, 1923b [5 5 species]. Type species Dendrothripoides ipomoeae Bagnall, 1923b (=Euthrips innoxius Karny, 1914b), by monotypy.

Dentothrips Faure, 1933 [1 1 species]. Type species Dentothrips graminis Faure, 1933, by monotypy.

Desertathrips de Borbón, 2008 [1 species]. Type species Desertathrips chuquiraga de Borbón, 2008, by monotypy.

Deuterobrachythrips Schmutz, 1913 [1 species]. Type species Heliothrips (Deuterobrachythrips) lineata Schmutz,1913, by monotypy. 
Diarthrothrips Williams, 1915 [2 species]. Type species Diarthrothrips coffeae Williams, 1915, by monotypy.

Dichromothrips Priesner, 1932d [18 species]. Type species Dichromothrips orchidis Priesner, 1932d, by monotypy.

Eugeneothrips Hood, 1938a. Type species Taeniothrips (Eugeneothrips) priesneri Hood, 1938a, by monotypy. Synonymy by Pitkin \& Mound (1973).

Dictyothrips Uzel, 1895 [1 1 species]. Type species Dictyothrips betae Uzel, 1895, by monotypy.

Dikrothrips Mound \& Walker, 1982 [1 species] Type species Dikrothrips diphyes Mound \& Walker, 1982, by monotypy.

Dodonaeathrips Mound \& Masumoto, 2009 [1 species]. Type species Dodonaeathrips eremiae Mound \& Masumoto, 2009, by monotypy.

Doonthrips Bhatti, Veer \& Chauhan, 1994 [1 species]. Type species Doonthrips setor Bhatti, Veer \& Chauhan, 1994, by monotypy.

Drepanothrips Uzel, 1895 [1 1 species]. Type species Drepanothrips reuteri Uzel, 1895, by monotypy.

Dyseringothrips zur Strassen, 1993 [1 1 species]. Type species Dyseringothrips vanharteni zur Strassen,1993, by monotypy.

Echinothrips Moulton, 1911 [7 species]. Type species Echinothrips mexicanus Moulton, 1911, by monotypy.

Enneothrips Hood, 1935b [5 species]. Type species Enneothrips gustaviae Hood, 1935b, by monotypy.

Enneothrips (Enneothripiella) Moulton, 1941: 318. Type species Enneothrips (Enneothripiella) flavens Moulton, 1941, by monotypy. Synonymy by Mound \& Marullo (1996).

$\dagger$ Eochirothrips Schliephake, 1999 [1 species]. Type species Eochirothrips mirocornis Schliephake, 1999, by monotypy.

Ephedrothrips zur Strassen, 1968a [2 species]. Type species Ephedrothrips maroccanus zur Strassen, 1968 a, by monotypy.

Ereikithrips Knechtel, 1960 [1 species]. Type species Ereikithrips calcaratus Knechtel, 1960, by monotypy.
Eremiothrips Priesner, 1950a [21 species]. Type species Eremiothrips imitator Priesner, 1950a, by monotypy.

Ascirtothrips Priesner, 1965. Type species Ascirtothrips efflatouni Priesner, 1965, by original designation. Synonymy by Bhatti (1988b).

Ascirtothrips (Antilopothrips) Priesner, 1965. Type species Anaphothrips antilope Priesner, 1965, by original designation. Synonymy by Bhatti (1988b).

Ernothrips Bhatti, 1967a [4 species]. Type species Thrips (Ernothrips) lobatus Bhatti, 1967a, by monotypy.

Eryngyothrips Bhatti, 1979a [6 species]. Type species Oxythrips eryngi Priesner, 1946, by original designation.

Euphysothrips Bagnall, 1926e [2 species]. Type species Euphysothrips minozzii Bagnall, 1926e, by monotypy.

Megaphysothrips Ramakrishna \& Margabandhu, 1939. Type species Megaphysothrips subramanii Ramakrishna \& Margabandhu, 1939, by monotypy. Synonymy by Shumsher (1946).

Exothrips Priesner, 1939c [20 species]. Type species Exothrips monstrosus Priesner, 1939c, by monotypy.

Anaphothrips (Dantabahuthrips) Shumsher, 1942. Type species Anaphothrips (Dantabahuthrips) sacchari Shumsher, 1942, by monotypy. Synonymy by Bhatti (1970a).

Catina Faure, 1956. Type species Catina papyri Faure, 1956, by monotypy. Synonymy by Bhatti (1970a).

Pexothrips Bhatti, 1967a. Type species Pexothrips anolis Bhatti, 1967a, by monotypy. Synonymy by Bhatti (1970a).

Ficothrips Minaei, 2012 [1 1 species]. Type species Ficothrips moundi Minaei, 2012, by monotypy.

Filipinothrips Reyes, 1994 [2 species]. Type species Filipinothrips baltazarae Reyes, 1994, by monotypy.

Firmothrips Schliephake, 1972 [1 1 species]. Type species Oxythrips firmus Uzel, 1895, by subsequent designation of Bhatti (1978c).

Flavidothrips Bhatti, 1978b [1 species]. Type species Anaphothrips flavidus Hood, 1925b, by monotypy. 
Florithrips Bhatti, 1970a [2 species]. Type species Physapus traegardhi Trybom, 1911, by monotypy.

Foliothrips Bhatti, 1972 [1 1 species]. Type species Foliothrips oratus Bhatti, 1972, by monotypy.

Frankliniella Karny, 1910 [236 species]. Type species Thrips intonsa Trybom, 1895, by subsequent designation of Hood (1914b).

Exophthalmothrips Moulton, 1933a. Type species Exophthalmothrips longipennis Moulton, 1933a, by original designation. Synonymy by Mound \& Marullo (1996).

Bolbothrips Crawford, 1945. Type species Bolbothrips aztecus Crawford, 1945, by original designation. Synonymy by Beshear (1982).

Fulmekiola Karny, 1925c [1 species]. Type species Fulmekiola interrupta Karny, 1925c (=Thrips serrata Kobus, 1892), by original designation.

Thrips (Saccharothrips) Priesner, 1934b. Type species Thrips serrata Kobus, 1892, by monotypy. Synonymy by Priesner (1938c).

Furcathrips Bournier \& Bournier, 1988 [1 species]. Type species Furcathrips bicolor Bournier \& Bournier, 1988, by monotypy.

Furcithrips Bhatti, 1984 [2 species]. Type species Furcithrips moundi Bhatti, 1984, by original designation.

Gabanithrips Bhatti \& Mound, 1992 [1 species]. Type species Gabanithrips bantus Bhatti \& Mound, 1992, by monotypy.

Glaucothrips Karny, 1921a [1 species]. Type species Pseudothrips glaucus Bagnall, 1914a, by monotypy.

Gnomonothrips Ramakrishna \& Margabandhu, 1939 [1 species]. Type species Gnomonothrips coimbatorensis Ramakrishna \& Margabandhu, 1939, by monotypy.

Gonzalezya Retana-Salazar, 2007b [1 1 species]. Type species Gonzalezya marinae Retana-Salazar, 2007b, by monotypy.

Guerothrips Goldarazena \& Infante, 2013 [1 species]. Type species Guerothrips moundi Goldarazena \& Infante, 2013, by original designation.

Helenothrips zur Strassen, 1976 [1 1 species]. Type species Helenothrips tinctus zur Strassen, 1976, by monotypy.
Hemianaphothrips Priesner, 1925a [1 Type species Anaphothrips (Hemianaphothrips) articulosus Priesner, 1925a, by monotypy.

Hengduanothrips Han \& Cui, 1992 [1 species]. Type species Hengduanothrips chrysis Han \& Cui, 1992, by monotypy.

Himalthrips Bhatti, 1979c [1 species]. Type species Taeniothrips janetscheki Pelikán, 1970, by monotypy.

Hyalopterothrips Priesner, 1939a [4 species]. Type species Anaphothrips (Hyalopterothrips) crocatus Priesner, 1939a, by original designation.

Idolimothrips Priesner, 1920a [2 species]. Type species Idolimothrips paradoxus Priesner, 1920a, by monotypy.

$\dagger$ Incertothrips Schliephake, 2000 [2 species]. Type species Incertothrips hoffeinsorum Schliephake, 2000 , by original designation.

Indusiothrips Priesner, 1952a [2 species]. Type species Indusiothrips seshadrii Priesner, 1952a, by monotypy.

Iridothrips Priesner, 1940a [5 species]. Type species Bregmatothrips iridis Watson, 1924, by monotypy.

Pelikanothrips Bhatti, 1978c. Type species Taeniothrips kratochvili Pelikán, 1947, by monotypy. Synonymy by Wang et al. (2019).

Isunidothrips Kudo, 1992 [1 1 species]. Type species Isunidothrips serangga Kudo, 1992, by monotypy.

Jakthrips Bhatti \& Ranganath, 2006 [1 species]. Type species Jakthrips ignacimuthui Bhatti \& Ranganath, 2006, by monotypy.

Kakothrips Williams, 1914 [8 species]. Type species Physopus robusta Uzel, 1895 (=Thrips pisivorus Westwood, 1880), by monotypy.

Prometheothrips Savenko, 1941. Type species Prometheothrips ungulatus Savenko, 1941, by monotypy. Synonymy by zur Strassen (1996).

Karphothrips Mound \& Walker, 1982 [1 1 species]. Type species Karphothrips dugdalei Mound \& Walker,1982, by monotypy.

Kenyattathrips Mound, 2009a [1 species]. Type species Kenyattathrips katarinae Mound, 2009a, by monotypy. 
Konothrips Bhatti, 1990a [3 species]. Type species Chirothrips tuttleyi zur Strassen, 1967, by monotypy.

Kranzithrips Mound, 2011 [1 species]. Type species Kranzithrips mareebai Mound, 2011, by monotypy.

Krokeothrips zur Strassen, 1995 [1 species]. Type species Taeniothrips innocens Priesner, 1922a, by monotypy.

Kurtomathrips Moulton, 1927c [4 species]. Type species Kurtomathrips morrilli Moulton, 1927c, by monotypy.

Laplothrips Bhatti, 1972 [2 species]. Type species Laplothrips bicolor Bhatti, 1972, by monotypy.

Larothrips Pitkin, 1973 [1 1 species]. Type species Larothrips dentipes Pitkin, 1973, by monotypy.

Lefroyothrips Priesner, 1938d [5 species]. Type species Physothrips lefroyi Bagnall, 1913b, by original designation.

†Lewisothrips Schliephake, 1993 [1 1 species]. Type species Lewisothrips telothripoides Schliephake, 1993, by monotypy.

Limothrips Haliday, 1836 [8 species]. Type species Thrips (Limothrips) cerealium Haliday, 1836, by subsequent designation of Westwood (1838).

Pruthiella Shumser Singh, 1942. Type species Limothrips angulicornis Jablonowski, 1894, by monotypy. Synonymy by zur Strassen (2003).

Albertothrips Shumser Singh, 1942 - nomen nudum - error for Pruthiella.

†Lipsanothrips Priesner, 1929a [1 1 species $]$. Type species Lipsanothrips skwarrae Priesner, 1929a, by monotypy.

Lomatothrips Mound \& Walker, 1982 [2 species]. Type species Lomatothrips paryphis Mound \& Walker, 1982 , by monotypy.

Longothrips Bhatti, 1990a [1 species]. Type species Chirothrips longiceps Strassen, 1969, by monotypy.

Macrurothrips Vuillet, 1914a [1 species]. Type species Macrurothrips normandi Vuillet, 1914a, by monotypy.

Masamithrips Mound, 2011 [6 species]. Type species Masamithrips masamii Mound, 2011, by original designation.
Mecothrips Karny, 1921b [1 species]. Type species Mecothrips anomoceras Karny, 1921b, by subsequent designation of Priesner (1938d).

Megalurothrips Bagnall, 1915b [14 species]. Type species Megalurothrips typicus Bagnall, 1915b, by monotypy.

Taeniothrips (Pongamiothrips) Ananthakrishnan, 1962. Type species Physothrips peculiaris Bagnall, 1918, by monotypy. Synonymy by Bhatti (1969b).

Lundathrips Bournier, 1979. Type species Lundathrips inopinatus Bournier, 1979, by monotypy. Synonymy by Bhatti (1990b).

Metaxyothrips Priesner, 1933c [2 species]. Type species Metaxyothrips exspectatus Priesner, 1933c, by monotypy.

Microcephalothrips Bagnall, 1926c [1 species]. Type species Thrips abdominalis Crawford, 1910, by monotypy.

Paraphysopus Girault, 1927. Type species Paraphysopus burnsi Girault, 1927, by monotypy. Synonymy by Medina-Gaud (1961).

Stylothrips Karny, 1926. Type species Stylothrips brevipalpus Karny, 1926, by monotypy. Synonymy by Priesner (1949b).

Ctenothripiella Priesner, 1927a. Type species Thrips abdominalis Crawford, 1910, by subsequent designation. Synonymy by Priesner (1927a).

Aureothrips Raizada, 1966. Type species Aureothrips marigoldae Raizada, 1966 (=Thrips abdominalis Crawford, 1910), by monotypy. Synonymy by Bhatti (1969a).

Monothrips Moulton, 1940 [2 species]. Type species Monothrips flavus Moulton, 1940, by monotypy.

Moundinothrips Bhatti, 1999b [1 species]. Replacement name for Moundothrips Bhatti, 1995a, not Moundothrips Wilson, 1975.

Moundothrips Bhatti, 1995a. Type species Moundothrips robustus Bhatti, 1995a, by monotypy.

Muscithrips Bournier, 1990 [3 species]. Type species Muscithrips obesus Bournier, 1990, by original designation.

Mycterothrips Trybom, 1910 [37 species $]$. Type species Mycterothrips laticauda Trybom, 1910, by monotypy. 
Physothrips Karny, 1912a. Type species by original designation Thrips ulmifoliorum Haliday, 1836, misidentification of Thrips salicis Reuter, 1879, by subsequent designation of Mound \& O’Neill (1969). Synonymy by Bhatti (1970a).

Taeniothrips (Rhopalandrothrips) Priesner, 1922b. Type species Thrips (Euthrips) consociata TargioniTozzetti, 1887, by subsequent designation of Priesner (1925d). Synonymy by Bhatti (1970a).

Pseudoscirtothrips Bournier, 1979. Type species Pseudoscirtothrips imbimbiachetae Bournier, 1979, by monotypy. Synonymy by Bhatti (1990b).

Neocorynothrips Ramakrishna \& Margabandhu, 1939 [1 species]. Type species Neocorynothrips asiaticus Ramakrishna \& Margabandhu, 1939, by monotypy.

Neurisothrips Sakimura, 1967 [14 species]. Type species Thrips multispinus Bagnall, 1910b, by original designation.

Nexothrips Marullo \& Mound, 2001 [2 species]. Type species Nexothrips delclaroi Marullo \& Mound, 2001, by original designation.

Nigritothrips Bhatti, 1978b [2 species]. Type species Belothrips longistylosus Priesner, 1920b, by original designation.

Octothrips Moulton, 1940 [3 species]. Type species Octothrips suspensus Moulton, 1940, by monotypy.

Apollothrips Wilson, 1972. Type species Apollothrips bhattii Wilson, 1972, by monotypy. Synonymy by Bhatti (2003b).

Odontanaphothrips Moulton, 1926 [1 1 species]. Type species Anaphothrips tricolor Moulton, 1911, by monotypy.

Odontothripiella Bagnall, 1929a [18 species]. Type species Odontothripiella fasciatipennis Bagnall, 1929a, by original designation.

Odontothripoides Bagnall, 1929a. Type species Odontothripoides morisoni Bagnall, 1929a, by monotypy. Synonymy by Pitkin (1972).

Odontothrips Amyot \& Serville, 1843 [35 species]. Type species Thrips phaleratus Haliday, 1836, by subsequent designation of Karny (1907).

Oelschlaegera Bhatti, 1990a [4 species]. Type species Chirothrips madagassus zur Strassen, 1961, by monotypy.
Okajimaella Nonaka \& Jangvitaya, 1994 [3 species]. Type species Okajimaella clara Nonaka \& Jangvitaya, 1994, by original designation.

Organothrips Hood, 1940 [6 species]. Type species Organothrips bianchii Hood, 1940, by monotypy.

Graminothrips Zhang \& Tong, 1992. Type species Graminothrips cyperi Zhang \& Tong, 1992, by original designation. Synonymy by Zhang et al. (2018).

Oxyrrhinothrips Priesner, 1933c [2 species]. Type species Thrips (Oxyrrhinothrips) scolopax Priesner, 1933c, by monotypy.

Oxythrips Uzel, 1895 [46 species]. Type species Oxythrips ajugae Uzel, 1895, by subsequent designation of Hood (1916d).

Protoxythrips Priesner, 1929a. Type species Oxythrips divisus Hood, 1916, by subsequent designation of Priesner (1949b). Synonymy by Stannard (1968a).

Retamothrips Bhatti, 1978b. Type species Anaphothrips retamae Priesner, 1934a, by monotypy. Synonymy by zur Strassen (2000).

Microxythrips Johansen \& Mojica, 1989. Type species Oxythrips mexicanus Johansen, 1983b, by monotypy. Synonymy by Mound \& Marullo (1996).

Chilothrips Hood, 1916b. Type species Chilothrips pini Hood, 1916b, by monotypy. Synonymy by Zhang et al. (2018).

Ozanaphothrips Mound \& Masumoto, 2009 [6 species]. Type species Ozanaphothrips fenarius Mound \& Masumoto, 2009, by original designation.

Paithrips Nonaka \& Jangvitaya, 1994 [2 species]. Type species Paithrips circularis Nonaka \& Jangvitaya, 1994, by monotypy.

Palmiothrips Bhatti, 1978b [1 species]. Type species Hemianaphothrips palmae Ramakrishna, 1934, by monotypy.

Pandanothrips Masumoto, Ng \& Okajima, 2013 [3 species]. Type species Pandanothrips ryukyuensis Masumoto, Ng \& Okajima, 2013, by original designation.

Pandorathrips Mound \& Masumoto, 2009 [1 species]. Type species Pandorathrips ascius Mound \& Masumoto, 2009, by monotypy.

Parabaliothrips Priesner, 1935b [6 species]. Type species Parabaliothrips takahashii Priesner, 1935b, by original designation. 
Krasibothrips Kudo, 1977. Type species Krasibothrips coluckus Kudo, 1977, by monotypy. Synonymy by Bhatti (1979c).

Yehiella Chen, 1977. Type species Yehiella aequosetosa Chen, 1977, by monotypy. Synonymy by Bhatti (1990b).

Paraleucothrips Johansen, 1983b [1 species]. Type species Paraleucothrips minusculus Johansen, 1983 b, by monotypy.

Parascirtothrips Masumoto \& Okajima, 2007 [1 species]. Type species Parascirtothrips fagaceae Masumoto \& Okajima, 2007, by monotypy.

Parexothrips Priesner, 1965 [3 species]. Type species Exothrips tenellus Priesner, 1950a, by monotypy.

Paroxythrips Masumoto \& Okajima, 2017 [2 species]. Type species Paroxythrips podocarpae Masumoto \& Okajima, 2017, by original designation.

Pezothrips Karny, 1907 [13 species]. Type species Physopus frontalis Uzel, 1895, by monotypy.

Yaothrips Mirab-balou et al., 2011. Type species Yaothrips shii Mirab-balou et al., 2011, by monotypy. Synonymy by Tong \& Liu (2019).

Physemothrips Stannard, 1963a [2 species]. Type species Physemothrips chrysodermus Stannard, 1963a, by monotypy.

Platythrips Uzel, 1895 [1 species]. Type species Thrips tunicata Haliday, 1895, by monotypy.

Plesiothrips Hood, 1915e [17 species]. Type species Sericothrips perplexa Beach, 1897, by monotypy.

Ornothrips Ananthakrishnan, 1965. Type species Thrips panicus Moulton, 1929b, by original designation. Synonymy by Ananthakrishnan (1969a).

Plutonothrips Priesner, 1933c [2 species]. Type species Plutonothrips cuspidatus Priesner, 1933c, by monotypy.

Arathrips Bhatti, 1967a. Type species Arathrips cus Bhatti, 1967a, by monotypy. Synonymy by Bhatti (1984).

Priesneriola Ananthakrishnan, 1964a [1 species]. Type species Priesneriola oneillae Ananthakrishnan, $1964 a$, by monotypy.

Prionotothrips Hood, 1954c [1 species]. Type species Prionotothrips procerus Hood, 1954c, by monotypy.
$†$ Procerothrips Bagnall, 1924 [1 species]. Type species Procerothrips cylindricornis Bagnall, 1924, by monotypy.

Projectothrips Moulton, 1929a [9 5 species]. Type species Projectothrips pruthii Moulton, 1929a, by monotypy.

Docidothrips Priesner, 1933c. Type species Docidothrips demonstrans Priesner, 1933c, by monotypy. Synonymy by Bhatti (1970a).

Stulothrips Moulton, 1934. Type species Stulothrips trespinus Moulton, 1934, by monotypy. Synonymy by Bhatti (1970a).

Proscirtothrips Karny, 1921a [1 species]. Type species Anaphothrips zeae Moulton, 1911, by subsequent designation of Priesner (1949b).

Prosopoanaphothrips Moulton, 1926 [1 species]. Type species Sericothrips reticulatus Moulton, 1907, by monotypy.

Prosopothrips Uzel, 1895 [9 species]. Type species Prosopothrips vejdovskyi Uzel, 1895, by monotypy.

$\dagger$ Protanaphothrips Schliephake, 2001b [12 species]. Type species Anaphothrips minoricornis Schliephake, 1999, by original designation.

Psectrothrips Hood, 1937a [7 species]. Type species Psectrothrips delostomae Hood, 1937a, by monotypy.

Lacandonithrips Johansen, 1981a. Type species Lacandonithrips elegantis Johansen, 1981a, by monotypy. Synonymy by Palmer \& Mound (1985).

Quirosiella Retana, 2000. Type species Quirosiella sotoi Retana, 2000, by original designation. Synonymy by Goldarazena et al. (2008).

Pseudanaphothrips Karny, 1921a [9 species]. Type species Pseudothrips achaetus Bagnall, 1916b, by subsequent designation of Karny (1924).

Homochaetothrips Sakimura, 1968. Type species Pseudothrips parvus Bagnall, 1916a, by original designation. Synonymy by Mound \& Palmer (1981).

Pseudothrips Hinds, 1902 [6 species]. Type species Thrips inaequalis Beach, 1897, by monotypy.

Schistothrips Hood, 1954c. Type species Schistothrips quadratus Hood, 1954c, by monotypy. Synonymy by Palmer \& Mound (1985). 
Linneothrips Johansen \& Mojica, 1996. Type species Linneothrips bicolor Johansen \& Mojica, 1996, by monotypy. Synonymy by Goldarazena et al. (2008).

Pseudoxythrips Priesner, 1940b [2 species]. Type species Oxythrips dentatus Knechtel, 1923, by monotypy.

Psilothrips Hood, 1927b [5 species]. Type species Psilothrips pardalotus Hood, 1927b, by monotypy.

Thamnothrips Priesner, 1932a. Type species Thamnothrips bimaculatus Priesner, 1932a, by monotypy. Synonymy by Priesner (1949b).

Psydrothrips Palmer \& Mound, 1985 [2 species]. Type species Psydrothrips kewi Palmer \& Mound, 1985, by monotypy.

Pteridothrips Priesner, 1938d [1 species]. Type species Physothrips pteridicola Karny, 1915a, by monotypy.

Ranjana Bhatti, 1978a [1 species]. Type species Euthrips cingulatus Karny, 1913c, by monotypy.

Retanathrips Mound \& Nickle, 2009 [5 species]. Type species Physothrips funestus Hood, 1915c, by original designation.

Rhabdothrips Hood, 1933a [1 species]. Type species Rhabdothrips albus Hood, 1933a, by monotypy.

Rhamphiskothrips Mound, 1990 [1 1 species]. Type species Rhamphiskothrips rhipistos Mound, 1990, by monotypy.

Rhamphothrips Karny, 1913c [20 species]. Replacement name for Rhynchothrips Karny, not Rhynchothrips Hood.

Rhynchothrips Karny, 1912c. Type species Rhynchothrips tenuirostris Karny, 1912c, by monotypy.

Perissothrips Hood, 1919b. Type species Perissothrips parviceps Hood, 1919b, by monotypy. Synonymy by Bhatti (1978d).

Perissothrips (Bdalsidothrips) Priesner, 1936c. Type species Perissothrips (Bdalsidothrips) levis Priesner, 1936c, by monotypy. Synonymy by Bhatti (1978d).

Pyctothrips Priesner, 1939c. Type species Pyctothrips albizziae Priesner, 1939c, by monotypy. Synonymy by Bhatti (1978d).

Rhaphidothrips Uzel, 1895 [1 species]. Type species Rhaphidothrips longistylosus Uzel, 1895, by monotypy.

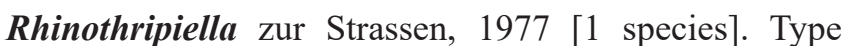
species Rhinothripiella ctenifera zur Strassen, 1977, by monotypy.

Rhinothrips Faure, 1933 [1 1 species]. Type species Rhinothrips rostratus Faure, 1933, by monotypy.

Rubiothrips Schliephake, 1975 [10 species]. Type species Anaphothrips ferruginea Uzel, 1895, by original designation.

Salpingothrips Hood, 1935b [3 species]. Type species Salpingothrips minimus Hood, 1935b, by monotypy.

†Saxonothrips Schliephake, 1999 [1 1 species]. Type species Taeniothrips (Saxonothrips) saxonicus Schliephake, 1999, by original designation.

Sciothrips Bhatti, 1970a [1 1 species]. Type species Taeniothrips cardamomi Ramakrishna, 1935, by monotypy.

Scirtidothrips Hood, 1954c [1 species]. Type species Scirtidothrips torquatus Hood, 1954c, by monotypy.

Scirtothrips Shull, 1909 [106 species]. Type species Scirtothrips ruthveni Shull, 1909, by monotypy.

Sericothripoides Bagnall, 1929c. Type species Dendrothrips bispinosus Bagnall, 1924a, by monotypy. Synonymy by Hood (1935b).

Sericopsothrips Hood, 1936a. Type species Sericopsothrips palloris Hood, 1936a, by monotypy. Synonymy by Ng \& Mound (2015).

Labiothrips Bhatti \& Mound, 1994. Type species Labiothrips tenor Bhatti \& Mound, 1994, by monotypy. Synonymy by Hoddle \& Mound (2003).

Scolothrips Hinds, 1902 [16 species]. Type species Thrips sexmaculata Pergande, 1894 by monotypy.

Chaetothrips Schille, 1911. Type species Chaetothrips uzeli Schille, 1911, by monotypy. Synonymy by Bagnall (1914b).

Siamothrips Okajima, 1990 [3 species]. Type species Siamothrips argus Okajima, 1990, by monotypy.

Simulothrips Nonaka \& Jangvitaya, 1994 [1 1 species]. Type species Simulothrips banpoti Nonaka \& Jangvitaya, 1994, by monotypy.

Sitothrips Priesner, 1931b [5 5 species]. Type species Sitothrips arabicus Priesner, 1931b, by monotypy.

Astenothrips Savenko, 1941. Type species Astenothrips georgicus Savenko, 1941, by monotypy. Synonymy by zur Strassen (2000). 
Smeringothrips Priesner, 1938d [1 5 species]. Type species Smeringothrips salaciae Priesner, 1938d, by monotypy.

Smilothrips Bhatti, 1976 [1 1 species]. Type species Smilothrips productus Bhatti, 1976, by monotypy.

Sminyothrips Uzel, 1895 [2 species]. Type species Sminyothrips biuncinatus Uzel, 1895, by subsequent designation of Priesner (1925: 150).

Solanithrips Johansen, 1997 [3 species]. Type species Bravothrips halffteri Johansen, 1986, by original designation.

Sorghothrips Priesner, 1936c [5 species]. Type species Thrips longistylus Trybom, 1911, by monotypy.

Ramakrishnothrips Shumser, 1942. Type species Taeniothrips jonnaphilus Ramakrishna, 1928, by original designation. Synonymy by Bhatti (1970).

Pellothrips Ananthakrishnan, 1969a. Type species Ornothrips fuscus Ananthakrishnan, 1965a, by monotypy. Synonymy by Bhatti (1970a).

Sphaeropothrips Priesner, 1928c [1 1 species]. Type species Sphaeropothrips inauditus Priesner, 1928c, by monotypy.

Ednathrips Bhatti, 1976. Type species Ednathrips pardus Bhatti, 1976, by monotypy. Synonymy by Bhatti \& Mound (1981).

Stannardia zur Strassen, 1959 [1 species]. Type species Stannardia chipingae zur Strassen, 1959, by monotypy.

Stenchaetothrips Bagnall, 1926c [42 species]. Type species Stenchaetothrips melanurus Bagnall, 1926c, by monotypy.

Anaphidothrips Hood, 1954c. Type species Anaphidothrips brasiliensis Hood, 1954c, by monotypy. Synonymy by Bhatti \& Mound (1981).

Chloethrips Priesner, 1957. Type species Thrips (Bagnallia) oryzae Williams, 1916, by original designation. Synonymy by Bhatti \& Mound (1981).

Chloethrips (Mictothrips) Ananthakrishnan \& Jagadish, 1967. Invalid, type species not designated. Synonymy by Bhatti \& Mound (1981).

Chloethrips (Xynothrips) Ananthakrishnan \& Jagadish, 1967. Invalid, type species not designated. Synonymy by Bhatti \& Mound (1981).

Stenothrips Uzel, 1895 [1 species]. Type species Stenothrips graminum Uzel, 1895, by monotypy.
Bagnallia Karny, 1910. Type species Bagnallia capito Karny, 1910, by monotypy. Synonymy by Priesner (1928a).

Striathrips Mound, 2011 [1 1 species]. Type species Striathrips sulcatus Mound, 2011, by monotypy.

Synaptothrips Trybom, 1910 [11 species]. Type species Synaptothrips crassicornis Trybom, 1910, by monotypy.

Homothrips Bagnall, 1915b. Type species Homothrips distinctus Bagnall, 1915b, by monotypy. Synonymy by Mound (1968b).

Limphysothrips Bagnall, 1919. Type species Limphysothrips paradoxus Bagnall, 1919, by monotypy. Synonymy by Mound (1968b).

Systenothrips Hood, 1956b [1 species]. Type species Systenothrips latens Hood, 1956b, by monotypy.

Taeniothrips Amyot \& Serville, 1843 [32 species]. Type species Thrips primulae Haliday, 1836 (=Thrips picipes Zetterstedt, 1828), by subsequent designation of Karny (1907).

Taeniothrips (Fetothrips) Bhatti, 1995b. Type species Taeniothrips tigris Bhatti, 1995b, by monotypy.

Javathrips Bhatti, 1978c. Type species Taeniothrips lagoenifer Priesner, 1938d, by original designation. Synonymy by Mirab-Balou et al. (2015).

Takethrips Nonaka \& Jangvitaya, 1993 [2 species]. Type species Takethrips bambusae Nonaka \& Jangvitaya, 1993, by original designation.

Tamaricothrips Priesner, 1964 [1 1 species]. Type species Anaphothrips tamaricis Bagnall, 1926, by monotypy.

Tameothrips Bhatti, 1978b [2 species]. Type species Euthrips tamicola Bagnall, 1914c, by monotypy.

†Telothrips Priesner, 1929a [2 species]. Type species Telothrips klebsi Priesner, 1929a, by original designation

Tenothrips Bhatti, 1967a [20 species]. Type species Taeniothrips (Tenothrips) alis Bhatti, 1967a, by monotypy.

Bournieriana Bhatti, 1995b. Type species Taeniothrips brevis Bournier, 1969, by original designation. Synonymy by zur Strassen (2000).

Gelbothrips Bhatti, 1995b. Type species Taeniothrips latoides Pelikán, 1968, by monotypy. Synonymy by Bhatti (2003a). 
Ewartithrips Nakahara, 1996a. Type species Euthrips ehrhornii Moulton, 1907, by original designation. Synonymy by Bhatti (2003a).

$\dagger$ Tethysthrips Nel et al., 2010 [2 species]. Type species Tethysthrips hispanicus Nel et al., 2010, by original designation.

Theilopedothrips zur Strassen, 1995 [1 species]. Type species Physopus pilosus Uzel, 1895, by monotypy.

Pilathrips Bhatti, 1995b. Type species Physopus pilosus Uzel, 1895, by original designation. Synonymy by Bhatti (1999b).

Thermothripoides Pelikán, 1984 [1 species]. Type species Thermothripoides zurstrasseni Pelikán, 1984, by monotypy.

Thermothrips Pelikán, 1949 [1 species]. Type species Anaphothrips (Thermothrips) mohelensis Pelikán, 1949, by monotypy.

Thrips Linnaeus, 1758 [300 species]. Type species Thrips physapus Linnaeus, 1758, by subsequent designation of Latreille (1810).

Euthrips Targioni-Tozzetti, 1887. Type species Thrips physapus Linneaeus, 1758, by inference (Hood 1914b). Synonymy by Hood (1914b).

Parathrips Karny, 1907. Type species Parathrips uzeli Karny, 1907, by monotypy. Synonymy by Jacot-Guillarmod (1975).

Achaetothrips Karny, 1908. Type species Achaetothrips loboptera Karny, 1908, by monotypy. Synonymy by Jacot-Guillarmod (1975).

Archaeothrips Field, 1910. Nomen nudum, lapsus for Achaetothrips Karny, 1908.

Isoneurothrips Bagnall, 1915b. Type species Isoneurothrips australis Bagnall, 1915b, by original designation. Synonymy by Bhatti (1980).

Parafrankliniella Priesner, 1920a. Type species Parafrankliniella verbasci Priesner, 1920a, by monotypy. Synonymy by Mound et al. (1976).

Paulus Solowiow, 1924. Type species Paulus gracilis Solowiow, 1924, by monotypy. Synonymy by JacotGuillarmod (1975).

Thrips (Epithrips) Priesner, 1926b. Type species Thrips (Epithrips) uzelianus Priesner, 1926b, by monotypy. Synonymy by Priesner (1964).

Ramaswamiahiella Karny, 1926. Type species Ramaswamiahiella subnudula Karny, 1926, by monotypy. Synonymy by Mound \& Masumoto (2005).

Priesneria Maltbaek, 1928. Type species Priesneria mancosetosa Maltbaek, 1928, by monotypy. Nomen nudum.

Isochaetothrips Moulton, 1928b. Type species Thrips seticollis Bagnall, 1915b, by original designation. Synonymy by Bhatti (1978c).

Anomalothrips Morgan, 1929. Type species Anomalothrips amygdali Morgan,1929 (=Thrips australis Bagnall, 1915b), by monotypy. Synonymy by Priesner (1964).

Thrips (Athrips) Priesner, 1938b. Type species Thrips kikuyuensis brevisetosa Trybom, 1912b, by monotypy. Synonymy by Mound \& Masumoto (2005).

Peladothrips Priesner, 1940b. Type species Peladothrips biunculatus Priesner, 1940b, by monotypy. Synonymy by Bhatti (1990b).

Thrips (Isothrips) Priesner, 1940b. Type species Isoneurothrips orientalis Bagnall, 1915, by monotypy. Synonymy by Mound \& Palmer (1981).

Kemothrips Bhatti, 1967a. Type species Kemothrips mirus Bhatti, 1967a, by monotypy. Synonymy by Mound \& Masumoto (2005).

Taeniothrips (Similothrips) Schliephake, 1972. Type species Thrips atratus Haliday, 1836, by original designation. Synonymy by Bhatti (1978c).

Thrips (Bidentothrips) Bournier \& Bournier, 1979. Type species Thrips (Bidentothrips) englerinae Bournier \& Bournier, 1979, by monotypy. Synonymy by Mound \& Masumoto (2005).

Jessicathrips Gonzalez, Retana-Salazar \& Castillo, 2010. Type species Jessicathrips cubensis Gonzalez et al., 2010. Nomen nudum, Synonymy by Mound (2015).

Tmetothrips Amyot \& Serville, 1843 [1 1 species]. Type species Thrips subaptera Haliday, 1836, by subsequent designation of Karny (1912a).

Pachythrips Uzel, 1895. Type species Thrips subaptera Haliday, 1836, by monotypy. Synonymy by Priesner (1925d)

Toxonothrips Moulton, 1927b [1 species]. Type species Toxonothrips gramineae Moulton, 1927b, by monotypy. 
Trachynotothrips Masumoto \& Okajima, 2005 [2 species]. Type species Trachynotothrips striatus Masumoto \& Okajima, 2005, by original designation.

Trichromothrips Priesner, 1930b [38 species]. Type species Trichromothrips bellus Priesner, 1930b, by monotypy.

Dorcadothrips Priesner, 1932b. Type species Dorcadothrips caespitis Priesner, 1932b, by monotypy. Synonymy by Bhatti (1999a).

Micothrips Ananthakrishnan, 1965a. Type species Micothrips fasciatus Ananthakrishnan,1965a, by monotypy. Synonymy by Bhatti (1999a).

Apothrips Bhatti, 1967a. Type species Apothrips priesneri Bhatti, 1967a, by monotypy. Synonymy by Ananthakrishnan \& Sen (1980).

Trichromothrips (Dovithrips) Bhatti, 1999a. Type species Dorcadothrips flavidus Bhatti, 1978e, by monotypy.

Tsutsumiothrips Masumoto \& Okajima, 2013 [1 species]. Type species Tsutsumiothrips ryukyuensis Masumoto \& Okajima, 2013, by original designation.

Tusothrips Bhatti, 1967a [4 species]. Type species Mycterothrips pseudosetiprivus Ramakrishna \& Margabandhu, 1939 (=Anaphothrips sumatrensis Karny, 1925c), by monotypy.

Unilobothrips Nakahara \& Foottit, 2012 [1 species]. Type species Unilobothrips cornuatus Nakahara \& Foottit, 2012, by monotypy.

Vulgatothrips Han, 1997 [3 species]. Type species Vulgatothrips shennongjiaensis Han, 1997, by monotypy.

Watanabeothrips Okajima, 2002 [1 1 species]. Type species Watanabeothrips yasuakii Okajima, 2002, by monotypy.

Wegenerithrips Johansen, 1983b [9 species]. Type species Wegenerithrips admirabilis Johansen, 1983b, by monotypy.

Xerothrips Nakahara, 1996b [1 species]. Type species Xerothrips dissimilis Nakahara, 1996b, by monotypy.

Yaobinthrips Zhang, Mound \& Xie, 2010 [1 species]. Type species Yaobinthrips yangtzei Zhang, Mound \& Xie, 2010, by monotypy.

Yoshinothrips Kudo, 1985 [4 species]. Type species Yoshinothrips pasekamui Kudo, 1985, by original desgination.
Zurstrassenia Bhatti, 1978b [1 species]. Type species Anaphothrips (Neophysopus) figuratus zur Strassen, 1968a, by monotypy.

Family $\uparrow$ Triassothripidae Grimaldi \& Shmakov ( 2 extinct genera)

$†$ Kazachothrips Shmakov, in Grimaldi, Shmakov \& Fraser, 2004 [1 species]. Type species Kazachothrips triassicus Shmakov, 2004, by monotypy.

$\uparrow$ Triassothrips Grimaldi \& Fraser, in Grimaldi, Shmakov \& Fraser, 2004 [1 species]. Type species Triassothrips virginicus Grimaldi \& Fraser, 2004, by monotypy.

\section{Family Uzelothripidae Hood}

(1 extant genus)

Uzelothrips Hood, 1952 [2 species]. Type species Uzelothrips scabrosus Hood, 1952, by monotypy.

Sub-Order Tubulifera Haliday

(1 extant family; 1 extinct family)

\section{Family $†$ Rohrthripidae Ulitzka}

(1 extinct genus)

$†$ Rohrthrips Nel et al., $2010[7$ species]. Type species Rohrthrips libanicus $\mathrm{Nel}$ et al., 2010, by monotypy.

Family Phlaeothripidae Haliday

(2 sub-families; 457 extant genera; 11 extinct genera)

Sub-family Idolothripinae Bagnall

(82 extant genera; no extinct genera)

Acallurothrips Bagnall, 1921a [22 species]. Type species Acallurothrips macrurus Bagnall, 1921a, by original designation.

Diopsothrips Hood, 1934a. Type species Diopsothrips flavus Hood, 1934a, by original designation. Synonymy by Mound \& Palmer (1983a).

Actinothrips Bagnall, 1909f [13 species]. Type species Actinothrips longicornis Bagnall, 1909f, by monotypy.

Dasythrips Hood, 1937b. Type species Dasythrips regalis Hood, 1937b, by monotypy. Synonymy by Mound \& Palmer (1983a). 
Aesthesiothrips Ananthakrishnan, 1961a [1 species]. Type species Aesthesiothrips jatrophae Ananthakrishnan, $1961 \mathrm{a}$, by monotypy.

Allidothrips zur Strassen, 1968a [2 species]. Type species Allidothrips tricolor zur Strassen, 1968a, by monotypy.

Allopisothrips Sakimura \& Bianchi, 1977 [1 species]. Type species Allopisothrips alakaiensis Sakimura \& Bianchi, 1977, by monotypy.

Allothrips Hood, 1908a [25 species]. Type species Allothrips megacephalus Hood, 1908a, by monotypy.

Bryothrips Priesner, 1925a. Type species Bryothrips pillichellus Priesner, 1925a, by monotypy. Synonymy by Stannard (1957).

Anactinothrips Bagnall, 1909f [15 species]. Type species Anactinothrips meinerti Bagnall, 1909f, by monotypy.

Ophidothrips Schmutz, 1909. Type species Ophidothrips handlirschii Schmutz, 1909, by monotypy. Synonymy by Moulton (1933d).

Lophothrips Karny, 1911b. Type species Lophothrips antennatus Karny, 1911b, by monotypy. Synonymy by Moulton (1933).

Chimairathrips Retana-Salazar, 2009. Type species Anactinothrips gustaviae Mound \& Palmer, 1983a, by monotypy. Status uncertain but listed under Anactinothrips in ThripsWiki (2021)

Amazoniella Retana-Salazar, 2009. Type species Anactinothrips gibbifer zur Strassen, 1980b, by monotypy. Status uncertain but listed under Anactinothrips in ThripsWiki (2021)

Incanothrips Retana-Salazar, 2009. Type species Anactinothrips graphidura Hood, 1938f, by original designation. Status uncertain but listed under Anactinothrips in ThripsWiki (2021)

Amerindiothrips Retana-Salazar, 2009. Type species Amerindiothrips gonzalezi Retana-Salazar, 2009, by original designation. Status uncertain but listed under Anactinothrips in ThripsWiki (2021)

Anaglyptothrips Mound \& Palmer, 1983a [1 species]. Type species Anaglyptothrips dugdalei Mound \& Palmer, 1983a, by monotypy.

Anallothrips Okajima \& Urushihara, 1997 [1 species]. Type species Anallothrips vietotubus Okajima \& Urushihara, 1997, by monotypy.
Atractothrips Hood, 1938b [2 species]. Type species Atractothrips bradleyi Hood, 1938b, by monotypy.

Azeugmatothrips Mound \& Palmer, 1983a [2 species]. Type species Azeugmatothrips rectus Mound \& Palmer, 1983a, by original designation.

Bacillothrips Buffa, 1908 [3 species]. Type species Bacillothrips linearis Buffa, 1908 (=Megalothrips longiceps Reuter, 1901), by monotypy.

Bactrothrips Karny, 1912d [53 species]. Type species Bactrothrips longiventris Karny, 1912d, by monotypy.

Eidothrips Bagnall, 1918. Type species Eidothrips alluaudi Bagnall, 1918, by monotypy. Synonymy by Mound \& Palmer (1983a).

Krinothrips Bagnall, 1918. Type species Krinothrips divergens Bagnall, 1918, by monotypy. Synonymy by Bagnall (1921c).

Bactridothrips Karny, 1919. Type species Bactridothrips idolomorphus Karny, 1919, by monotypy. Synonymy by Mound \& Palmer (1983a).

Caudothrips Karny, 1921a. Type species Caudothrips buffai Karny, 1921a, by monotypy. Synonymy by Mound \& Palmer (1983a).

Bactrianothrips Bagnall, 1936. Type species Bactrianothrips alluaudi Bagnall, 1936, by monotypy. Synonymy by Bournier (1968).

Cervothrips Bagnall, 1936. Type species Cervothrips berlandi Bagnall, 1936, by monotypy. Synonymy by Mound \& Palmer (1983a).

Lasiothrips Moulton, 1968. Type species Lasiothrips perplexus Moulton, 1968, by monotypy. Synonymy by Mound \& Tree (2011).

Bolothrips Priesner, 1926c [17 species]. Type species Phloeothrips bicolor Heeger, 1852b, by original designation.

Bolothrips (Botanothrips) Hood, 1939. Type species Bolothrips pratensis Hood, 1939, by original designation. Synonymy by Mound (1974c).

Boloadelothrips Moulton, 1949. Type species Boloadelothrips africanus Moulton, 1949, by monotypy. Synonymy by Mound \& Palmer (1983a).

Camachothrips Retana-Salazar, 2009 [2 species]. Type species Camachothrips diabolica Retana-Salazar, 2009 , by original designation. 
Campulothrips Moulton, 1944 [1 1 species]. Type species Campulothrips gracilis Moulton, 1944, by monotypy.

Carientothrips Moulton, 1944 [23 species]. Type species Bolothrips (Carientothrips) fijiensis Moulton, 1944, by monotypy.

Celidothrips Priesner, 1951 [4 species]. Type species Docessissophothrips adiaphorus Karny, 1923, by monotypy.

Ommatidothrips Mound, 1970a. Type species Ommatidothrips lawrencei Mound, 1970a, by monotypy. Synonymy by Mound (1974c).

Ceuthothrips Hood, 1938f [1 species]. Type species Ceuthothrips timuqua Hood, 1938f, by monotypy.

Cleistothrips Bagnall, 1932b [1 species]. Type species Cleistothrips idolothripoides Bagnall, 1932b, by monotypy.

Compsothrips Reuter, 1901 [27 species]. Type species Phloeothrips albosignata Reuter, 1884, by monotypy.

Macrothrips Buffa, 1908. Type species Phloeothrips albosignatus Reuter, 1884, by monotypy. Synonymy by Bagnall (1909a).

Leurothrips Bagnall, 1908c. Type species Leurothrips albomaculata Bagnall, 1908c, by original designation. Synonymy by Priesner (1928a).

Leptogastrothrips Trybom, 1913. Type species Leptogastrothrips reuteri Trybom, 1913, by monotypy. Synonymy by Priesner (1964).

Oedaleothrips Hood, 1916c. Type species Oedaleothrips hookeri Hood, 1916c, by original designation. Synonymy by Priesner (1964).

Myrmecothrips Watson, 1920. Type species Myrmecothrips querci Watson, 1920, by original designation. Synonymy by Hood (1936b).

Myrmecothrips Priesner, 1926d. Type species Myrmecothrips dampfi Priesner, 1926d, by original designation. Synonymy by Hood (1936b).

Formicothrips Priesner, 1927a. Replacement name for Myrmecothrips Priesner. Synonymy by Hood (1936b)

Cryptothrips Uzel, 1895 [12 species]. Type species Cryptothrips lata Uzel, 1895 (=Phloeothrips nigripes Reuter, 1880a), by subsequent designation of Hood (1916)
Cylindrothrips Moulton, 1949 [1 species]. Type species Cylindrothrips niger Moulton, 1949, by monotypy.

Cyphothrips Hood, 1952 [1 1 species]. Type species Cyphothrips dorsalis Hood, 1952, by monotypy.

Dermothrips Bagnall, 1910b [1 species]. Type species Dermothrips hawaiiensis Bagnall, 1910b, by monotypy.

Diaphorothrips Karny, 1920b [5 species]. Type species Diaphorothrips unguipes Karny, 1920b, by monotypy.

Diaphorothrips (Cnemidothrips) Priesner, 1940a. Type species Diaphorothrips hamipes Karny, 1923, by original designation.

Diceratothrips Bagnall, 1908c [18 species]. Type species Diceratothrips bicornis Bagnall, 1908c, by monotypy.

Eulophothrips Schmutz, 1909. Type species Eulophothrips robustus Schmutz, 1909, by monotypy. Synonymy by Priesner (1949b).

Megalomerothrips Watson, 1919. Type species Megalomerothrips eupatorii Watson, 1919, by monotypy. Synonymy by Priesner (1949b).

Diceratothrips (Endacnothrips) Priesner, 1933b. Type species Diceratothrips (Endacnothrips) horridus Priesner, 1933b, by monotypy. Synonymy by Mound \& Marullo (1996).

Dichaetothrips Hood, 1914c [4 species]. Type species Dichaetothrips brevicollis Hood, 1914c, by monotypy.

Dinothrips Bagnall, 1908c [6 species]. Type species Dinothrips sumatrensis Bagnall, 1908c, by monotypy.

Paxillothrips Ananthakrishnan, 1961a. Type species Paxillothrips longicauda Ananthakrishnan, 1961a, by monotypy. Synonymy by Palmer \& Mound (1978).

Diplacothrips Hood, 1937b [2 species]. Type species Diplacothrips borgmeieri Hood, 1937b, by monotypy.

Ecacleistothrips Mound, 2007 [1 species]. Type species Ecacleistothrips gloriosus Mound, 2007, by monotypy.

Egchocephalothrips Bagnall, 1916b [1 species]. Type species Docessissophothrips monstrosus Bagnall, 1909 e, by monotypy. 
Elaphrothrips Buffa, 1909 [144 species]. Type species Idolothrips coniferarum Pergande, 1896, by subsequent designation of Andre (1940).

Dicaiothrips Buffa, 1909. Type species Thrips schottii Heeger, 1852a, by subsequent designation of Bagnall (1910a). Synonymy by Hood (1927a).

Klinothrips Bagnall, 1918. Type species Klinothrips femoralis Bagnall, 1918, by monotypy. Synonymy by Priesner (1952b).

Elaphrothrips (Elaphroxothrips) Bagnall, 1932b. Type species Kleothrips athletes Karny, 1923, by monotypy.

Elaphridothrips Priesner, 1932e. Type species Elaphridothrips andrapterus Priesner, 1932e, by monotypy. Synonymy by Priesner (1952b).

Palinothrips Hood, 1952. Type species Palinothrips palustris Hood, 1952, by monotypy. Synonymy by Mound \& Palmer (1983a).

Elaphrothrips (Paraclinothrips) Priesner, 1952b Type species Elaphrothrips (Paraclinothrips) coniger Priesner, 1952b, by monotypy.

Elaphrothrips (Cradothrips) Ananthakrishnan, 1973b. Type species Elaphrothrips (Cradothrips) insignis Ananthakrishnan, 1973b, by monotypy.

Elgonima zur Strassen, 1972 [1 species]. Type species Elgonima seticeps zur Strassen, 1972, by monotypy.

Emprosthiothrips Moulton, 1942 [6 species]. Type species Emprosthiothrips niger Moulton, 1942, by monotypy.

Ethirothrips Karny, 1925a [37 species]. Type species Liothrips thomasseti Bagnall, 1921a (=Phlaeothrips stenomelas Walker, 1859), by subsequent designation of Priesner (1949b).

Scotothrips Priesner, 1939b. Type species Adiaphorothrips elephas Karny, 1920c, by original designation. Synonymy by Mound \& Palmer (1983a).

Paracryptothrips Moulton, 1944. Type species Paracryptothrips inermis Moulton, 1944, by original designation. Synonymy by Mound \& Palmer (1983a).

Percipiothrips Ananthakrishnan, 1964d. Type species Mesothrips indicus Bagnall, 1921b, by monotypy. Synonymy by Mound \& Palmer (1983a).

Elaphridia Ananthakrishnan, 1964d. Type species Elaphrothrips agasthya Ramakrishna, 1934, by monotypy. Synonymy by Mound \& Palmer (1983a).
Eurynotothrips Moulton, 1968. Type species Eurynotothrips latapennis Moulton, 1968, by monotypy. Synonymy by Mound \& Palmer (1983a).

Uredothrips Ananthakrishnan, 1969b. Type species Uredothrips indicus Ananthakrishnan, 1969b, by monotypy. Synonymy by Mound \& Palmer (1983a).

Decothrips Ananthakrishnan, 1969b. Type species Decothrips anacardii Ananthakrishnan, 1969b, by monotypy. Synonymy by Mound \& Palmer (1983a).

Faureothrips Priesner, 1949b [1 species]. Type species Cryptothrips reticulatus Trybom, 1913, by monotypy.

Gastrothrips Hood, 1912e [38 species]. Type species Gastrothrips ruficauda Hood, 1912e, by original designation.

Goetothrips Priesner, 1925c. Type species Goetothrips terrestris Priesner, 1925c, by monotypy. Synonymy by Johansen (1979b).

Isopterothrips Bagnall, 1926d. Type species Isopterothrips tenuipennis Bagnall, 1926d, by monotypy. Synonymy by Mound \& Palmer (1983a).

Syncerothrips Hood, 1935a. Type species Syncerothrips harti Hood, 1935a, by monotypy. Synonymy by Mound \& Palmer (1983a).

Probolothrips Moulton, 1941. Type species Probolothrips hambletoni Moulton, 1941 (=Gastrothrips abditus Hood, 1935a), by monotypy. Synonymy by Hood (1952).

Pharetrothrips Priesner, 1952a. Type species Agnostochthona curvidens Karny, 1921c, by monotypy. Synonymy by Mound \& Palmer (1983a).

Percnothrips Ananthakrishnan, 1967. Type species Percnothrips turbinatus Ananthakrishnan, 1967, by monotypy. Synonymy by Mound \& Palmer (1983a).

Paragastrothrips zur Strassen, 1977. Type species Paragastrothrips mauli zur Strassen, 1977, by monotypy. Synonymy by Mound \& Palmer (1983a).

Neodichaetothrips Johansen, 1982a. Type species Neodichaetothrips jalisciensis Johansen, 1982a, by monotypy. Synonymy by Mound \& Marullo (1996).

Hartwigia Faure, 1949a [1 species]. Type species Hartwigia tumiceps Faure, 1949a, by monotypy.

Heptathrips Moulton, 1942 [8 species]. Type species Heptathrips tonnoiri Moulton, 1942, by monotypy. 
Ascania Faure, 1954. Type species Ascania magnifica Faure, 1954, by monotypy. Synonymy by Mound \& Palmer (1983a).

Capnothrips zur Strassen, 1979. Type species Capnothrips ruficaudis zur Strassen, 1979, by monotypy. Synonymy by Mound \& Palmer (1983a).

Herathrips Mound, 1974a [1 species]. Type species Adiaphorothrips nativus Girault, 1928b, by monotypy.

Holurothrips Bagnall, 1914d [4 species]. Type species Holurothrips ornatus Bagnall, 1914d, by monotypy.

Hybridothrips Stannard, 1954 [1 species]. Type species Actinothrips (Hybridothrips) oneillae Stannard, 1954, by monotypy.

Hystricothrips Karny, 1912d [2 species]. Type species Hystricothrips phasgonura Karny, 1912d, by monotypy.

Zeugmatothripoides Bagnall, 1929c. Type species Zeugmatothripoides africanus Bagnall, 1929c, by monotypy. Synonymy by Mound (1968a).

Idolothrips Haliday in Walker, 1852 [3 species]. Type species Idolothrips marginatus Haliday, 1852 (=Idolothrips spectrum Haliday, 1852), by subsequent designation of Bagnall (1908b).

Acanthinothrips Bagnall, 1908c. Type species Idolothrips spectrum Haliday, 1852, by monotypy. Synonymy by Bagnall (1916).

Illinothrips Stannard, 1953a [1 species]. Type species Illinothrips rossi Stannard, 1953a, by monotypy.

Ischyrothrips Schmutz, 1913 [1 species]. Type species Ischyrothrips crassus Schmutz, 1913, by subsequent designation of Priesner (1949b).

Lamillothrips Bagnall, 1923b [3 species]. Type species Lamillothrips typicus Bagnall, 1923b, by monotypy.

Hylothrips Priesner, 1932e. Type species Hylothrips aethiopicus Priesner, 1932e, by original designation. Synonymy by Mound \& Palmer (1983a).

Loyolaia Ananthakrishnan, 1964b [3 species]. Type species Loyolaia indica Ananthakrishnan, 1964b, by monotypy.

Machatothrips Bagnall, 1908c [14 species]. Type species Machatothrips biuncinatus Bagnall, 1908c, by monotypy.
Adiaphorothrips Bagnall, 1909e. Type species Adiaphorothrips simplex Bagnall, 1909e, by original designation. Synonymy by Priesner (1939b).

Cnestrothrips Priesner, 1932e. Type species Cnestrothrips dammermani Priesner, 1932e, by original designation. Synonymy by Palmer \& Mound (1978).

Macrothrips Bagnall, 1908b [1 species]. Type species Macrothrips papuensis Bagnall, 1908b, by original designation.

Malesiathrips Palmer \& Mound, 1978 [4 species]. Type species Malesiathrips malayensis Palmer \& Mound, 1978 , by original designation.

Mecynothrips Bagnall, 1908b [14 species]. Type species Mecynothrips wallacei Bagnall, 1908b, by monotypy.

Phoxothrips Karny, 1913b. Type species Phoxothrips pugilator Karny, 1913b, by monotypy. Synonymy by Palmer \& Mound (1978)

Kleothrips Schmutz, 1913. Type species Kleothrips gigans Schmutz, 1913, by monotypy. Synonymy by Palmer \& Mound (1978).

Dracothrips Bagnall, 1914b. TypespeciesDracothrips ceylonicus Bagnall, 1914b, by monotypy. Synonymy by Palmer \& Mound (1978).

Acrothrips Karny, 1920c. Type species Acrothrips sorex Karny, 1920c, by monotypy. Synonymy by Palmer \& Mound (1978)

Kleothrips (Synkleothrips) Priesner, 1935a. Type species Kleothrips (Synkleothrips) innocens Priesner, 1935a, by monotypy. Synonymy by Palmer \& Mound (1978).

Kleothrips (Akleothrips) Priesner, 1935a. Type species Kleothrips (Akleothrips) karimonensis Priesner, 1935a, by monotypy. Synonymy by Palmer \& Mound (1978).

Megalothrips Uzel, 1895 [8 species]. Type species Megalothrips bonannii Uzel, 1895, by subsequent designation of Bagnall (1909a).

Megathrips Targioni-Tozzetti, 1881 [7 species]. Type species Megathrips piccioli Targioni-Tozzetti, 1881 (=Phloeothrips lativentris Heeger, 1852), by monotypy.

Siphonothrips Buffa, 1908. Type species Siphonothrips elegans Buffa, 1908, by monotypy. Synonymy by Mound \& Palmer (1983a). 
Meiothrips Priesner, 1929c [5 species]. Type species Idolothrips (Meiothrips) annulatus Priesner, 1929c (=Acanthinothrips annulipes Bagnall, 1914d), by monotypy.

Meiothrips (Aculeathrips) Kudo, 1975. Type species Meiothrips (Telothrips) nepalensis Kudo \& Ananthakrishnan, by monotypy. [Replacement name for Meiothrips (Telothrips) Kudo \& Ananthakrishnan, 1974 nec Telothrips Priesner, 1929a]. Synonymy by Palmer \& Mound (1978).

Minaeithrips Mound, 2007 [2 species]. Type species Minaeithrips aliceae Mound, 2007, by original designation.

Neatractothrips Mound \& Palmer, 1983a [1 species]. Type species Atractothrips macrurus Okajima, 1975, by monotypy.

Neosmerinthothrips Schmutz, 1913 [21 species]. Type species Neosmerinthothrips fructuum Schmutz, 1913, by monotypy.

Coenurothrips Bagnall, 1921a. Type species Coenurothrips brevicollis Bagnall, 1921a, by original designation. Synonymy by Mound (1974c).

Galactothrips Moulton, 1933d. Type species Galactothrips diversicolor Moulton, 1933d, by monotypy. Synonymy by Mound (1974c).

Nesidiothrips Mound, 1974c [2 species]. Type species Nesothrips alius Ananthakrishnan, 1970b, by original designation.

Nesothrips Kirkaldy, 1907 [31 species]. Type species Nesothrips oahuensis Kirkaldy, 1907, by monotypy.

Oedemothrips Bagnall, 1910b. Type species Oedemothrips laticeps Bagnall, 1910b, by monotypy. Synonymy by Bianchi (1944).

Rhaebothrips Karny, 1913b. Type species Rhaebothrips lativentris Karny, 1913b, by monotypy. Synonymy by Mound \& Palmer (1983a).

Ophthalmothrips Hood, 1919a [10 species]. Type species Ophthalmothrips pomeroyi Hood, 1919a, by monotypy.

Pyrgothrips Karny, 1924. Type species Pyrgothrips conocephalus Karny, 1924, by monotypy. Synonymy by Mound \& Palmer (1983a).

Fulgorothrips Faure, 1933. Type species Fulgorothrips priesneri Faure, 1933, by monotypy. Synonymy by Mound \& Palmer (1983a).
Derothrips Jacot-Guillarmod, 1940. Type species Derothrips amyae Jacot-Guillarmod, 1940, by monotypy. Synonymy by Mound \& Palmer (1983a).

Ozothrips Mound \& Palmer, 1983a [5 species]. Type species Ozothrips priscus Mound \& Palmer, 1983a, by original designation.

Paractinothrips Mound \& Palmer, 1983a [1 species]. Type species Paractinothrips peratus Mound \& Palmer, 1983a, by monotypy.

Pelinothrips Mound, 1974a [2 species]. Type species Rhopalothrips ornatus Girault, 1930, by original designation.

Peltariothrips Mound \& Palmer, 1983a [1 species]. Type species Peltariothrips insolitus Mound \& Palmer, 1983a, by monotypy.

Phacothrips Mound, 1974c [1 species]. Type species Gastrothrips ocelloides Hood, 1950, by monotypy.

Phaulothrips Hood, 1918 [20 species]. Type species Phaulothrips vuilleti Hood, 1918, by monotypy.

Titanothrips Karny, 1920c. Type species Titanothrips portentosus Karny, 1920c, by monotypy. Synonymy by Mound (1974a).

Tetraceratothrips Bagnall, 1924e. Type species Tetraceratothrips agrestis Bagnall, 1924e, by monotypy. Synonymy by Mound (1974a).

Kaleidothrips Kelly \& Mayne, 1934. Type species Kaleidothrips inquilinus Kelly \& Mayne, 1934, by monotypy. Synonymy by Mound \& Palmer (1983a).

Pinaceothrips Yakhontov, 1956 [1 species]. Type species Pinaceothrips monticola Yakhontov, 1956, by monotypy.

Polytrichothrips Priesner, 1939 [2 12 species]. Type species Polytrichothrips pilosus Priesner 1939 (=Docessissophothrips laticeps Bagnall, 1915a), by monotypy.

Priesneriana Ananthakrishnan, 1956b [3 species]. Type species Gnophothrips kabandha Ramakrishna, 1928, by monotypy.

Priesneriella Hood, 1927b [8 species]. Type species Priesneriella citricauda Hood, 1927b, by monotypy.

Pygidiothrips Hood, 1938d. Type species Pygidiothrips seminole Hood, 1938d, by monotypy. Synonymy by Mound \& Palmer (1983a). 
Parallothrips Hood, 1939. Type species Parallothrips thomasi Hood, 1939, by monotypy. Synonymy by Mound \& Palmer (1983a).

Embothrips Dyadechko, 1961. Type species Embothrips tubversicolor Dyadechko, 1961, by monotypy. Synonymy by Dyadechko (1964).

Pseudocryptothrips Priesner, 1919b [3 species]. Type species Pseudocryptothrips meridionalis Priesner, $1919 b$, by monotypy.

Pseudoeurhynchothrips Moulton, 1949 [2 species]. Type species Pseudoeurhynchothrips bidens Moulton, 1949, by monotypy.

Pygothrips Hood, 1915a [17 species]. Type species Pygothrips rugicauda Hood, 1915a, by monotypy.

Barythrips Hood \& Williams, 1915. Type species Barythrips sculpticauda Hood \& Williams, 1915, by monotypy. Synonymy by Mound \& Palmer (1983a).

Diplochelaeothrips Moulton, 1944. Type species Diplochelaeothrips mikrommatos Moulton, 1944, by monotypy. Synonymy by Mound \& Palmer (1983a).

Saurothrips Hood, 1952 [1 1 species]. Type species Saurothrips assai Hood, 1952, by monotypy.

Sporothrips Hood, 1938d [1 species]. Type species Adiaphorothrips amplus Hood, 1925f, by monotypy.

Tarassothrips Mound \& Palmer, 1983a [2 species]. Type species Tarassothrips akritus Mound \& Palmer, 1983a, by monotypy.

Tiarothrips Priesner, 1935a [1 species]. Type species Kleothrips subramanii Ramakrishna, 1925, by monotypy.

Zactinothrips Hood, 1936c [2 species]. Type species Zactinothrips elegans Hood, 1936c, by monotypy.

Zeuglothrips Hood, 1936c [1 species]. Type species Zeuglothrips echinus Hood, 1936c, by monotypy.

Zeugmatothrips Priesner, 1925c [18 species]. Type species Zeugmatothrips hispidus Priesner, 1925c, by monotypy.

Apozeugmatothrips Retana-Salazar, 2009. Type species Zeugmatothrips borgmeieri Hood, 1949, by original designation. Status uncertain but listed under Zeugmatothrips in ThripsWiki (2021).

Plesiozeugmatothrips Retana-Salazar, 2009. Type species Zeugmatothrips mumbaca Hood, 1952, by monotypy. Status uncertain but listed under Zeugmatothrips in ThripsWiki (2021).
Sub-family Phlaeothripinae Haliday

(376 extant genera; 11 extinct genera)

Ablemothrips Ananthakrishnan, 1969d [3 species]. Type species Ablemothrips maxillatus Ananthakrishnan, 1969d, by monotypy.

Acaciothrips Priesner, 1965 [1 species]. Type species Gynaikothrips ebneri Karny, 1920a, by monotypy.

Acanthothrips Uzel, 1895 [13 species]. Type species Phloeothrips nodicornis Reuter, 1880a, by monotypy.

Notothrips Hood, 1933b. Type species Phloeothrips vittatus Hood, 1912a, by original designation. Synonymy by Stannard (1957)

Aclystothrips Ananthakrishnan, 1972a [2 species]. Type species Aclystothrips priesneri Ananthakrishnan, 1972a, by monotypy.

Acrosothrips Stannard, 1963b [1 species]. Type species Trichothrips assymetricus Watson, 1937a, by monotypy.

Adamantothrips Stannard, 1960 [1 species]. Type species Adamantothrips asarcopus Stannard, 1960, by monotypy.

Adelphothrips Priesner, 1953 [5 species]. Type species Gynaikothrips tristis Karny, 1916, by original designation.

Adraneothrips Hood, 1925c [76 species]. Type species Haplothrips tibialis Hood, 1914a, by original designation.

Hypothrips Priesner, 1933a. Type species Hypothrips desocellatus Priesner, 1933a, by monotypy. Synonymy by Stannard (1957).

Stigmothrips Ananthakrishnan, 1964c. Type species Stigmothrips limpidus Ananthakrishnan, 1964c, by original designation. Synonymy by Dang, Mound \& Qiao (2013).

Baphikothrips Mound, 1970a. Type species Baphikothrips antennatus Mound, 1970a, by original designation. Synonymy with Stigmothrips by Okajima (1976).

Adrothrips Moulton, 1942 [5 species]. Type species Adrothrips aureus Moulton, 1942, by monotypy.

Adurothrips Mound, 1995 [1 species]. Type species Adurothrips atopus Mound, 1995, by monotypy. 
Advenathrips Morris, Mound \& Schwarz, 2000 [1 species]. Type species Advenathrips inquilinus Morris, Mound \& Schwarz, 2000, by monotypy.

Aeglothrips Ananthakrishnan, 1969c [1 species]. Type species Aeglothrips denticulus Ananthakrishnan, 1969 c, by monotypy.

Aesthetothrips Hood, 1954g [1 species]. Type species Aesthetothrips tucuche Hood, 1954g, by monotypy.

Afrothrips Bournier, 1970 [1 1 species]. Type species Afrothrips testaceus Bournier, 1970, by monotypy.

Agnostochthona Kirkaldy, 1907 [1 species]. Type species Agnostochthona alienigera Kirkaldy, 1907, by monotypy.

Agrothrips Jacot-Guillarmod, 1939 [7 species]. Type species Agrothrips priesneri Jacot-Guillarmod, 1939, by monotypy.

Agynaikothrips Okajima, 2006 [2 species]. Type species Agynaikothrips okinawaensis Okajima, 2006, by original designation.

Ahamothrips Kumar, Tyagi \& Bhatti, 2007 [1 species]. Type species Ahamothrips maxima Kumar Tyagi \& Bhatti, 2007, by monotypy. [Nomen nudum as type species name not valid]

Aiganothrips Bhatti, 1991 [1 species]. Type species Gynaikothrips hystrix Bagnall, 1928b, by monotypy.

Akainothrips Mound, 1971 [34 species]. Type species Adiaphorothrips citritarsus Girault, 1928a, by monotypy.

Akarethrips Dang, Mound \& Qiao, 2014 [1 1 species]. Type species Akarethrips iotus Dang, Mound \& Qiao, 2014, by monotypy.

Akthethrips Mound, 1970c [1 species]. Type species Akthethrips strobus Mound, 1970c, by monotypy.

Alerothrips Bhatti, 1995c [3 species]. Type species Neurothrips indicus Ananthakrishnan, 1964, by monotypy.

Aleurodothrips Franklin, 1909 [1 species]. Type species Cryptothrips fasciapennis Franklin, 1908, by monotypy.

Microcanthothrips Bagnall, 1914b. Type species Cephalothrips spinosus Bagnall, 1909c (=Cryptothrips fasciapennis Franklin), by monotypy. Synonymy by Mound (1968a).
Alloiothrips Ananthakrishnan, 1964c [1 species]. Type species Alloiothrips nigrisetis Ananthakrishnan, 1964c, by monotypy.

Alocothrips Priesner, 1952a [1 species]. Type species Trichothrips hadrocerus Karny, 1926, by monotypy.

Amothrips Priesner, 1939 [1 species]. Type species Amothrips ghesquierei Priesner, 1939, by monotypy.

Amphibolothrips Buffa, 1909 [3 species]. Type species Amphibolothrips grassii Buffa, 1909, by monotypy.

Amynothrips O'Neill, 1968 [1 species]. Type species Amynothrips andersoni O'Neill, 1968, by monotypy.

Ananthakrishnana Bhatti, 1967a [1 species]. Type species Ananthakrishnana indica Bhatti, 1967a (=Haplothrips euphorbiae Priesner, 1931d), by monotypy.

Ananthakrishnanothrips Bournier, 1985 [1 species]. Type species Ananthakrishnanothrips polysciae Bournier, 1985, by monotypy.

Andrethrips Mound, 1974b [2 species]. Type species Andrethrips floydi Mound, 1974b, by monotypy.

Androthrips Karny, 1911c [12 species]. Type species Mesothrips melastomae Zimmermann, 1900, by monotypy.

Acutothrips Karny, 1915b. Type species Androthrips flavipes Schmutz, 1913, by monotypy. The genus is considered a nomen nudum, having never been described but published as "in litt. et sched.".

Apelaunothrips Karny, 1925b [40 species]. Replacement name for Ophidothrips Karny, 1925c, nec Ophidothrips Schmutz, 1909. Type species Ophidothrips medioflavus Karny, 1925c, by monotypy.

Baphothrips Priesner, 1933c. Type species Baphothrips tricolor Priesner, 1933c, by monotypy. Synonymy by Mound (1974a).

Aphlothrips Tyagi \& Kumar, 2006 [1 species]. Type species Aphlothrips viraktamathii Tyagi \& Kumar, 2006, by monotypy.

Apostlethrips Mound \& Minaei, 2006 [3 species]. Type species Apostlethrips apostus Mound \& Minaei, 2006 , by original designation.

Apterygothrips Priesner, 1933d [39 species]. Type species Apterygothrips haloxyli Priesner, 1933d, by monotypy. 
Araeothrips Ananthakrishnan, 1976a [3 species]. Type species Araeothrips longisetis Ananthakrishnan, 1976a, by monotypy.

Argothrips Mound \& Tree, 2017 [1 1 species]. Type species Argothrips ommatus Mound \& Tree, 2017, by monotypy.

Arrhenothrips Hood, 1919b [7 species]. Type species Arrhenothrips ramakrishnae Hood, 1919b, by monotypy.

Asemothrips Hood, 1919c [5 species]. Type species Asemothrips picturatus Hood, 1919c, by monotypy.

Empresmothrips Karny, 1920c. Type species Empresmothrips combustipes Karny, 1920c, by monotypy. Synonymy by Mound (1974a).

Coenothrips Bagnall, 1924e. Type species Coenothrips fallax Bagnall, 1924e, by monotypy. Synonymy by Mound (1971).

Eucoenothrips Bagnall, 1926d. Unnecessary replacement name for Coenothrips, see Mound (1967).

Asianthrips Okajima, 2006 [1 species]. Type species Asianthrips orientalis Okajima, 2006, by monotypy.

Athlibothrips Priesner, 1952a [4 species]. Type species Gynaikothrips fuscipes Karny, 1922b, by monotypy.

Aulothrips Priesner, 1950b [1 1 species]. Type species Aulothrips nubicus Priesner, 1950b, by monotypy.

Austrothrips Brethes, 1915 [1 species]. Type species Austrothrips verae Brethes, 1915, by monotypy.

Ayyarothrips Ananthakrishnan, 1972a [1 species]. Type species Ayyarothrips abstrusus Ananthakrishnan, 1972a, by monotypy.

Azaleothrips Ananthakrishnan, 1964c [38 species]. Type species Azaleothrips amabilis Ananthakrishnan, $1964 c$, by monotypy.

Baenothrips Crawford, 1948 [12 species]. Type species Baenothrips guatemalensis Crawford, 1948, by monotypy.

Verrucothrips Stannard, 1952a. Type species Amphibolothrips (Verrucothrips) caenosa Stannard, 1952a, by monotypy. Synonymy by Mound (1972b).

Ramachandraiella Ananthakrishnan, 1964c. Type species Ramachandraiella minuta Ananthakrishnan, 1964c, by monotypy. Synonymy by Mound (1972b).
Transithrips Bournier, 1963. Type species Transithrips asper Bournier, 1963, by monotypy. Synonymy by Mound (1972b).

Bournieria Ananthakrishnan, 1966a. Type species Bournieria indica Ananthakrishnan, 1966a, by monotypy. Synonymy by Mound (1972b).

Bagnalliella Karny, 1920c [9 species]. Type species Cephalothrips yuccae Hinds, 1902, by monotypy.

Bamboosiella Ananthakrishnan, 1957 [31 species]. Type species Bamboosiella bicoloripes Ananthakrishnan, 1957 , by monotypy.

Antillothrips Stannard, 1957. Type species Antillothrips graminatus Stannard, 1957, by monotypy. Synonymy by Okajima (1995).

Elatea Faure, 1957. Type species Elatea stannardi Faure, 1957, by monotypy. Synonymy by Pitkin (1977).

Xenothrips Ananthakrishnan, 1965b. Type species Xenothrips malabaricus Ananthakrishnan, 1965b, by monotypy. Synonymy by Pitkin (1976).

Bangithrips Mound \& Ng, 2018 [1 species]. Type species Bangithrips mei Mound \& Ng, 2018, by monotypy.

Bebelothrips Buffa, 1909 [1 species]. Type species Bebelothrips latus Buffa, 1909, by monotypy.

Blepharidothrips Hood, 1952 [1 species]. Type species Blepharidothrips sphaerops Hood, 1952, by monotypy.

Bocathrips Goldarazena, 2010 [1 1 species]. Type species Bocathrips okajimai Goldarazena, 2010, by monotypy.

Boothrips Priesner, 1949b [1 species]. Type species Boothrips singularis Priesner, 1949b, by monotypy.

Brachythrips Reuter, 1899 [2 species]. Type species Brachythrips flavicornis Reuter, 1899, by monotypy.

Bradythrips Hood \& Williams, in Hood, 1925c [6 species]. Type species Bradythrips hesperus Hood \& Williams, in Hood, 1925c, by monotypy.

Brakothrips Crespi, Morris \& Mound, 2004 [8 species]. Type species Brakothrips gillesi Crespi, Morris \& Mound, 2004, by monotypy.

Buffettithrips Mound \& Wells, 2015 [1 species]. Type species Buffettithrips rauti Mound \& Wells, 2015, by monotypy. 
Bunothrips Ananthakrishnan, 1969d [1 species]. Type species Bunothrips cruralis Ananthakrishnan, 1969d, by monotypy.

Byctothrips Ananthakrishnan, 1973c [1 species]. Type species Byctothrips ayyari Ananthakrishnan, 1973c, by monotypy.

Calamothrips Ananthakrishnan, 1967 [1 species]. Type species Calamothrips fastigiatus Ananthakrishnan, 1967, by monotypy.

Callithrips Bournier, 1969b [1 species]. Type species Callithrips bicolor Bournier, 1969b, by monotypy.

Callococcithrips Mound \& Wells, 2007 [2 species]. Type species Rhynchothrips fuscipennis Moulton, 1968, by original designation.

Carathrips Hood, 1950 [14 species]. Type species Trichothrips mediamericanus Hood, 1933, by original designation.

Carcinothrips Moulton, 1929c [2 species]. Type species Carcinothrips leai Moulton, 1929c, by monotypy.

Carissothrips Ananthakrishnan, 1964b [1 species]. Type species Carissothrips nigrescens Ananthakrishnan, 1964b, by monotypy.

Carius Faure, 1961 [1 species]. Type species Carius malgassus Faure, 1961, by monotypy.

Cartomothrips Stannard, 1962 [6 species]. Type species Cartomothrips browni Stannard, 1962, by original designation.

Cecidothrips Kieffer, 1908 [1 species]. Type species Cecidothrips bursarum Kieffer, 1908, by monotypy.

Cephalothrips Uzel, 1895 [8 species]. Type species Phloeothrips monilicornis Reuter, 1885, by monotypy.

†Cephenothrips Priesner, 1929a [1 species]. Type species Cephenothrips laticeps Priesner, 1929a, by monotypy.

Chaetokarnyia Priesner, 1952a [4 species]. Type species Cryptothrips tenuicornis Karny, 1912b, by monotypy.

Aliothrips Priesner, 1953. Type species Aliothrips elegantulus Priesner, 1953, by original designation. Synonymy by Bhatti (1993).

Chalepothrips Priesner, 1968 [1 species]. Type species Chalepothrips ardisiae Priesner, 1968, by monotypy. [The genus and species names were published with a query mark and are thus considered "conditional" and unavailable; see note in ThripsWiki (2021)]
Chamaeothrips Hood, 1954a [2 species]. Type species Chamaeothrips jucundus Hood, 1954a, by monotypy.

Chelaeothrips Karny, 1923 [2 species]. Type species Chelaeothrips annamensis Karny, 1923, by monotypy.

Chiraplothrips Priesner, 1931a [3 species]. Type species Haplothrips (Chiraplothrips) faureanus Priesner, 1931a, by monotypy.

Chiridothrips Ramakrishna \& Margabandhu, 1939 [3 species]. Type species Chiridothrips indicus Ramakrishna \& Margabandhu, 1939, by monotypy.

Chiridurothrips Okajima, 1981 [2 species $]$. Type species Chiridurothrips hisakoae Okajima, 1981, by monotypy.

Chirothripoides Bagnall, 1915c [6 species]. Type species Chirothripoides typicus Bagnall,1915c, by monotypy.

Chlarathrips Ananthakrishnan, 1967 [1 species]. Type species Chlarathrips tersus Ananthakrishnan, 1967, by monotypy.

Choleothrips Moulton, 1927a [2 5 species]. Type species Choleothrips geijerae Moulton, 1927a, by monotypy.

Chorithrips Hood, 1957 [2 species]. Type species Chorithrips heptatoma Hood, 1957, by original designation.

Chortothrips Hood, 1957 [1 species]. Type species Eurythrips valens Hood, 1950, by monotypy.

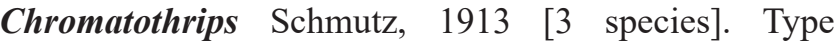
species Chromatothrips fasciatus Schmutz, 1913, by subsequent designation of Bagnall (1915a: 324), who also considered this species to be congeneric with Aleurodothrips Franklin.

Chthonothrips Hood, 1957 [1 species]. Type species Chthonothrips nigrocinctus Hood, 1957, by monotypy.

Claustrothrips Bournier, 1994 [1 species]. Type species Claustrothrips senegalensis Bournier, 1994, by monotypy.

Conocephalothrips Bianchi, 1946 [1 1 species]. Type species Conocephalothrips tricolor Bianchi, 1946, by monotypy.

Corroboreethrips Mound \& Moritz, 2000 [5 species]. Type species Corroboreethrips kallus Mound \& Moritz, 2000, by original designation. 
Corycidothrips Ananthakrishnan, 1972b [1 species]. Type species Corycidothrips inquilinus Ananthakrishnan, 1972 b, by monotypy.

Coryphothrips Karny, 1923 [2 species]. Type species Coryphothrips trochiceps Karny, 1923, by original designation.

Covidthrips Mound, 2020 [1 species]. Type species Covidthrips novendecim Mound, 2020, by monotypy.

Craniothrips Bagnall, 1915c [1 species]. Type species Craniothrips urichi Bagnall, 1915c, by monotypy.

Crespithrips Mound \& Morris, 2000 [2 species]. Type species Crespithrips enigmaticus Mound \& Morris, 2000 , by original designation.

Crinitothrips Okajima, 1978 [5 species]. Type species Crinitothrips setosus Okajima, 1978, by monotypy.

Crotonothrips Ananthakrishnan, 1968a [16 species]. Type species Crotonothrips gallarum Ananthakrishnan, 1968 a, by monotypy.

Phaeothrips Ananthakrishnan, 1969c. Type species Phaeothrips mimicus Ananthakrishnan, 1969c, by monotypy. Synonymy by Ananthakrishnan (1976b).

Crotonothrips (Inermothrips) Muraleedharan \& Sen, 1978. Type species Crotonothrips (Inermothrips) cacharensis Muraleedharan \& Sen, 1978, by monotypy.

Csirothrips Mound, 1971 [1 species]. Type species Csirothrips watsoni Mound, 1971, by monotypy.

Dactylothrips Bagnall, 1923b [31 species]. Type species Dactylothrips australis Bagnall, 1923b, by monotypy.

Hannibalia Girault, 1928b. Type species Hannibalia priscus Girault, 1928b, by monotypy. Synonymy by Mound (1969).

Deplorothrips Mound \& Walker, 1986 [23 species]. Type species Deplorothrips bassus Mound \& Walker, 1986, by monotypy.

Dexiothrips Hartwig, 1952 [1 species]. Type species Dexiothrips pensus Hartwig, 1952, by monotypy.

Dimorphothrips Bagnall, 1928b [2 species]. Type species Dimorphothrips microchaetus Bagnall, 1928b, by original designation.

Diphyothrips Stannard, 1963b [1 species]. Type species Diphyothrips morainensis Stannard, 1963b, by monotypy.
Dixothrips Ananthakrishnan, 1969d [1 species]. Type species Dixothrips onerosus Ananthakrishnan, 1969d, by monotypy.

Docessissophothrips Bagnall, 1908c [14 species]. Type species Docessissophothrips ampliceps Bagnall, $1908 \mathrm{c}$, by monotypy.

Polyphemothrips Schmutz, 1909. Type species Polyphemothrips brasiliensis Schmutz, 1909, by monotypy. Synonymy by Mound \& Palmer (1983a).

Dolicholepta Priesner, 1932e [11 species]. Type species Dolichothrips giraffa Karny, 1920a, by original designation.

Dolichothrips Karny, 1912c [21 species]. Type species Dolichothrips longicollis Karny, 1912c, by monotypy.

Membrothrips Bhatti, 1978f. Type species Neoheegeria indica Hood, 1919b, by monotypy. Synonymy by Mound \& Minaei (2007).

Domatiathrips Mound 1993 [1 species]. Type species Domatiathrips cunninghamii Mound, 1993, by monotypy.

Domeothrips Crespi, Morris \& Mound, 2004 [5 species]. Type species Domeothrips aruena Crespi, Morris \& Mound, 2004, by original designation.

Dopothrips Bhatti, 1995c [1 species]. Type species Podothrips paraensis Hood, 1952, by monotypy.

Drypetothrips Mound \& Wells, 2020 [1 species]. Type species Drypetothrips korykis Mound \& Wells, 2020, by monotypy.

Dunatothrips Moulton, 1942 [7 species]. Type species Dunatothrips armatus Moulton, 1942, by monotypy.

Dyscolothrips Mamet, 1967 [1 species]. Type species Dyscolothrips priesneri Mamet, 1967, by monotypy.

Ecacanthothrips Bagnall, 1909 [11 species]. Type species Acanthothrips sanguineus Bagnall, 1908b, by monotypy.

Ormothrips Buffa, 1909. Type species Ormothrips inermis Buffa, 1909, by monotypy. Synonymy by Bagnall (1911).

Eothrips Hood, 1915d [13 species]. Type species Dolerothrips crassicornis Karny, 1912b, by original designation.

Eparsothrips zur Strassen, 1968c [1 species]. Type species Podothrips varicornis Bagnall, 1919, by monotypy. 
Epiliothrips Priesner, 1965 [2 species]. Type species Gynaikothrips willcocksi Bagnall, 1921b, by original designation.

Epomisothrips Hood, 1954a [1 species]. Type species Epomisothrips araucariae Hood, 1954a, by monotypy.

Eschatothrips Stannard, 1955 [7 species]. Type species Eurythrips reticulotubus Stannard, 1953c, by original designation.

Eugynothrips Priesner, 1926c [16 species]. Type species Cryptothrips conocephali Karny, 1913c, by subsequent designation of Brothers \& Mound (1985).

Loepothrips Priesner, 1952a. Type species Dolerothrips coarctatus Karny, 1915d, by original designation. [Currently a sub-genus]

Eumorphothrips Schmutz, 1913 [1 1 species $].$ Type species Eumorphothrips albicornis Schmutz, 1913, by monotypy.

Euoplothrips Hood, 1918 [6 species]. Type species Euoplothrips bagnalli Hood, 1918, by monotypy.

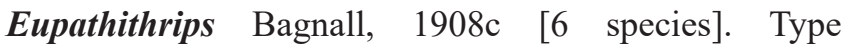
species Eupathithrips dentipes Bagnall, 1908c, by monotypy.

Heterothrips Buffa, 1908. Type species Heterothrips silvestrii Buffa, 1908, by monotypy. Synonymy by Priesner (1949b).

Polyommatothrips Buffa, 1909. Replacement name for Heterothrips Buffa nec Heterothrips Hood, 1908.

Eurhynchothrips Bagnall, 1918 [5 species]. Type species Eurhynchothrips convergens Bagnall, 1918, by monotypy.

Eurhynchothrips (Nephothrips) Priesner, 1949b. Type species Eurhynchothrips bipunctatus Priesner, 1939 , by monotypy.

Euryaplothrips Ramakrishna \& Margabandhu, 1931 [1 species]. Type species Euryaplothrips crassus Ramakrishna \& Margabandhu, 1931, by monotypy.

Eurynothrips Bagnall, 1908c [1 species]. Type species Eurynothrips magnicollis Bagnall, 1908c, by original designation.

Eurythrips Hinds, 1902 [39 species]. Type species Eurythrips ampliventralis Hinds, 1902, by original designation.
Plemmelothrips Hood, 1957. Type species Plemmelothrips defectus Hood, 1957, by monotypy. Synonymy by Mound (1977a).

Marinaella Retana-Salazar \& Soto-Rodríguez, 2013a. Type species Marinaella edwini RetanaSalazar \& Soto-Rodríguez, 2013a, by monotypy. Synonymy by Mound (2015).

Camilothrips Retana-Salazar \& Soto-Rodríguez, 2013b. Type species Camilothrips saidamhedi Retana-Salazar \& Soto-Rodríguez, 2013b, by monotypy. Synonymy by Mound (2015).

Eurytrichothrips Priesner, 1920a [1 1 species]. Type species Eurytrichothrips piniphilus Priesner, 1920a, by monotypy.

Fourbethrips Soto-Rodríguez, Nishida \& Retana-Salazar, 2012 [1 species]. Type species Fourbethrips fiorella Soto-Rodríguez, Nishida \& Retana-Salazar, 2012, by monotypy.

Gabonothrips Bournier, 1970 [1 species]. Type species Gabonothrips grassei Bournier, 1970, by monotypy.

Gemmathrips Reyes, 1994 [1 species]. Type species Gemmathrips brevis Reyes, 1994, by monotypy.

Gigantothrips Zimmermann, 1900 [19 species]. Type species Gigantothrips elegans Zimmermann, 1900, by monotypy.

Panurothrips Bagnall, 1908c. Type species Panurothrips gracilis Bagnall, 1908c, by monotypy. Synonymy by Bagnall (1916b).

Syringothrips Priesner, 1933c. Type species Syringothrips gallicola Priesner, 1933c, by monotypy. Synonymy by Dang et al. (2014).

Giraultithrips Mound \& Tree, 2014b [1 species]. Type species Bagnalliella nigricoxa Girault, 1929, by monotypy.

Glaridothrips Crespi, Morris \& Mound, 2004 [1 species]. Type species Glaridothrips koptus Crespi, Morris \& Mound, 2004, by monotypy.

Glenothrips Priesner, 1921b [1 species]. Type species Cryptothrips biuncinatus Karny, 1913c, by monotypy.

Glubothrips Ananthakrishnan, 1969d [1 species]. Type species Glubothrips mucidus Ananthakrishnan, 1969d, by monotypy.

Gluphothrips Moulton, 1944 [1 species]. Type species Gluphothrips varicolor Moulton, 1944, by monotypy. 
Glyptothrips Hood, 1912d [14 species]. Type species Glyptothrips flavescens Hood, 1912d, by monotypy.

Erkosothrips Stannard, 1955. Type species Erkosothrips interior Stannard, 1955, by original designation. Synonymy by Mound (1977a).

Gnophothrips Hood \& Williams, 1915 [1 species]. Type species Gnophothrips megaceps Hood \& Williams, 1915, by monotypy.

Godoythrips Mound \& Marullo, 1996 [1 species]. Type species Godoythrips asketus Mound \& Marullo, 1996, by monotypy.

Gomphiothrips Moulton, 1933d [2 species]. Type species Gomphiothrips tibouchinae Moulton, 1933d, by monotypy.

Goniothrips Hood, 1927b [1 species]. Type species Goniothrips denticornis Hood, 1927b, by monotypy.

Grypothrips Karny, 1924 [6 species]. Type species Grypothrips mantis Karny, 1924, by monotypy.

Gymnothrips Karny, 1911b [1 species]. Type species Gymnothrips moultoni Karny, 1911b, by monotypy.

Gynaikothrips Zimmermann, 1900 [42 species]. Type species Gynaikothrips uzeli Zimmermann, 1900, by monotypy.

Sauridothrips Priesner, 1953. Type species Sauridothrips phlaeothripinus Priesner, 1953, by monotypy. Synonymy by Dang et al. (2014).

Habrothrips Ananthakrishnan, 1968a [1 species]. Type species Habrothrips curiosus Ananthakrishnan, 1968a, by monotypy.

Hadothrips Priesner, 1925c [1 species]. Type species Haplothrips (Zygothrips) robinae Priesner, 1924a, by subsequent designation of Priesner (1949b).

Pezidothrips Priesner, 1951. Type species Haplothrips (Zygothrips) robinae Priesner, 1924, by monotypy. Synonymy by Bhatti (1993).

Halothrips Bournier, 1962 [1 species]. Type species Halothrips salicorniae Bournier, 1962, by monotypy.

Hansonthrips Mound \& Marullo, 1996 [2 species]. Type species Hansonthrips drymus Mound \& Marullo, 1996, by original designation.

Hapedothrips Bhatti, 1995c [1 species]. Type species Haplothrips trifolii angusticornis Priesner, 1921b, by monotypy.
Hapelothrips zur Strassen, 1972 [1 1 species]. Type species Hapelothrips albipes zur Strassen, 1972, by monotypy.

Haplothrips Amyot \& Serville, 1843 [240 species]. Type species Phloeothrips albipennis Burmeister, 1838, by monotypy.

Zygothrips Uzel, 1895. Type species Zygothrips minutus Uzel, 1895, by monotypy. Synonymy by Priesner (1928a).

Anthothrips Uzel, 1895. Type species Thrips aculeata Fabricius, 1803, by monotypy. Synonymy by Bagnall (1912).

Anthemothrips Karny, 1907. Type species Anthemothrips reuteri Karny, 1907, by monotypy. Synonymy by Priesner (1925d).

Hindsiana Karny, 1910. Type species Hindsiana flavicincta Karny, 1910, by monotypy. Synonymy by Priesner (1949b).

Porphyrothrips Vuillet, 1913. Type species Porphyrothrips cottei Vuillet, 1913, by monotypy. Synonymy by Priesner (1949b).

Zygothrips (Chonothrips) John, 1924. Type species Zygothrips (Chonothrips) crassicornis John,1924, by monotypy. Synonymy by Priesner (1928a).

Haplothrips (Trybomiella) Bagnall, 1926d. Type species Anthothrips bagnalli Trybom, 1910, by original designation.

Arrhenochiris Enderlein, 1929. Type species Arrhenochiris retamae Enderlein, 1929, by monotypy. Synonymy by Priesner (1931a).

Hapliothrips Bagnall, 1934. Type species Hapliothrips globiceps Bagnall, 1934, by monotypy. Synonymy by Priesner (1950b).

Haplothrips (Anchylothrips) Hood, 1939. Haplothrips (Anchylothrips) preeri Hood, 1939, by monotypy.

Haplothrips (Pallidothrips) Pelikán, 1963a. Type species Haplothrips (Pallidothrips) yakhontovi Pelikán, 1963a, by monotypy.

Segnothrips Ananthakrishnan, 1965b. Type species Segnothrips trivandrensis Ananthakrishnan, 1965b, by monotypy. Synonymy by Bhatti (1993).

Hexagoniothrips Johansen \& Oca, 1989. Type species Hexagoniothrips axochoensis Johansen \& Oca, 1989, by monotypy. Synonymy by Mound \& Marullo (1996). 
Jironiella Retana-Salazar \& Soto-Rodríguez, 2007. Type species Jironiella saidi Retana-Salazar \& Soto-Rodríguez, 2007, by monotypy. Synonymy by Goldarazena et al. (2008).

Dyothrips Kudo, 1974. Type species Haplothrips cingulatus Pelikán, 1963b, by monotypy. Synonymy by Tyagi et al. (2018).

Hapsidothrips Mamet, 1967 [1 species]. Type species Hapsidothrips curtispinis Mamet, 1967, by monotypy.

Heligmothrips Mound, 1970c [6 species]. Type species Trichothrips erinaceus Karny, 1920c, by original designation.

Heliothripoides Okajima, 1987b [2 species]. Type species Heliothripoides reticulatus Okajima, 1987b, by monotypy.

Heptadikothrips Crespi, Morris \& Mound, 2004 [1 species]. Type species Heptadikothrips thallus Crespi, Morris \& Mound, 2004, by monotypy.

Hexadikothrips Crespi, Morris \& Mound, 2004 [1 species]. Type species Hexadikothrips dalbyi Crespi, Morris \& Mound, 2004, by monotypy

Hindsiothrips Stannard, $1958 \quad$ [6 1 species $]$ Type species Hindsiana pullata Hood, 1925, by original designation.

Boloplothrips Titschack, 1957. Type species Watsoniella bonessi Titschack, 1955, by monotypy. Synonymy by Priesner 1964.

Hiplothrips Dyadechko, 1964 [1 species]. Type species Haplothrips zhigajevi Dyadechko, 1962, by monotypy.

Holcothrips Hood, 1954a [1 species]. Type species Holcothrips achmaeae Hood, 1954a, by monotypy.

Holoengythrips Mound \& Tree, 2014a [9 species]. Type species Holoengythrips maynardae Mound \& Tree, 2014a, by original designation.

Holopothrips Hood, 1914a [60 species]. Type species Holopothrips signatus Hood, 1914a, by original designation.

Phrasterothrips Priesner, 1921c. Type species Phrasterothrips conducans Priesner, 1921c, by monotypy. Synonymy by Mound \& Marullo (1996).

Diploacanthothrips Moulton, 1933b. Type species Diploacanthothrips fuscus Moulton, 1933b, by monotypy. Synonymy of Phrasterothrips by Priesner (1949b).
Homorothrips Hood, 1954a. Type species Homorothrips erianthi Hood, 1954a, by monotypy. Synonymy by Mound \& Marullo (1996).

Anoplothrips Hood, 1954a. Type species Anoplothrips jaboticabae Hood, 1954a, by monotypy. Synonymy by Mound \& Marullo (1996).

Caraibothrips Bournier, 1993. Type species Caraibothrips inquilinus Bournier, 1993, by monotypy. Synonymy by Mound \& Marullo (1996).

Holothrips Karny, 1911b [141 species]. Type species Holothrips ingens Karny, 1911b, by monotypy.

Abiastothrips Priesner, 1925d. Type species Trichothrips schaubergeri Priesner, 1920a, by original designation. Synonymy by Mound \& Marullo (1996).

Cratothrips Priesner, 1927a. Type species Cratothrips angulatus Priesner, 1927a, by monotypy. Synonym of Abiastothrips by Mound \& Palmer (1983a).

Lathrobiothrips Hood, 1934a. Type species Lathrobiothrips ramuli Hood, 1934a, by monotypy. Synonymy by Mound \& Palmer (1983a).

Cordylothrips Hood, 1937b. Type species Cordylothrips peruvianus Hood, 1937b, by monotypy. Synonymy by Mound \& Palmer (1983a).

Adelothrips Hood, 1938d. Type species Adelothrips xanthopus Hood, 1938d, by original designation. Synonymy by Mound \& Palmer (1983a).

Ischnothrips Moulton, 1944. Type species Ischnothrips zimmermanni Moulton, 1944, by monotypy. Synonymy by Mound \& Palmer (1983a).

Agnostothrips Moulton, 1947. Type species Agnostothrips semiflavus Moulton,1947, by monotypy. Synonymy by Mound \& Palmer (1983a).

Pseudosymphyothrips Kurosawa, 1954. Type species Pseudosymphyothrips yuasai Kurosawa, 1954, by monotypy. Synonymy by Okajima (1987a).

Erythrinothrips Ananthakrishnan, 1956a. Type species Agnostothrips (Erythrinothrips) indicus Ananthakrishnan, 1956a, by monotypy. Synonymy by Mound \& Palmer (1983a).

Stinothrips Ananthakrishnan, 1969e. Type species Ischnothrips typicus Ananthakrishnan, 1967, by monotypy. Synonymy by Mound \& Palmer (1983a).

Holmiella zur Strassen, 1972. Type species Holmiella nigrita zur Strassen, 1972, by monotypy. Synonymy by Mound \& Palmer (1983a). 
Kolia Soto-Rodríguez, Retana-Salazar \& Rodríguez Arrieta, 2013. Type species Kolia guanacastensis Soto-Rodríguez, Retana-Salazar \& Rodríguez Arrieta, 2013, by original designation. Synonymy by Mound (2015).

Arthrips Soto-Rodríguez, Retana-Salazar \& Rodríguez Arrieta, 2013. Type species Arthrips breedyi Soto-Rodríguez, Retana-Salazar \& Rodríguez Arrieta, 2013, by original designation. Synonymy by Mound (2015).

Hoodiana Faure, 1933 [1 species]. Type species Hoodiana pallida Faure, 1933, by monotypy.

Hoplandrothrips Hood, 1912e [117 species]. Type species Phloeothrips (Hoplandrothrips) xanthopus Hood, 1912e (=Phloeothrips jennei Jones, 1912), by original designation.

Phloeobiothrips Hood, 1925e. Phloeobiothrips tumiceps Hood, 1925e, by monotypy. Synonymy by Stannard (1957).

Cryptaplothrips Priesner, 1927a. Type species Cryptothrips (Cryptaplothrips) famelicus Priesner, 1927 a, by monotypy. Synonymy by Stannard (1957)

Pathothrips Hood, 1937b. Type species Hoplandrothrips (Pathothrips) proteus Hood, 1937b, by monotypy. Synonymy by Stannard (1957).

Gynoplothrips Priesner, 1949b. Type species Hoplandrothrips arrhenus Jacot-Guillarmod, 1939, by monotypy. Synonymy by Stannard (1957).

Hoplothrips Amyot \& Serville, 1843 [131 species] Type species Thrips corticis De Geer, 1773, by subsequent designation of Karny (1912a).

Trichothrips Uzel, 1895. Type species Phloeothrips pedicularia Haliday, 1836, by subsequent designation of Hood (1915d). Synonymy by Stannard (1957).

Dolerothrips Bagnall, 1910b. Type species Dolerothrips flavipes Bagnall, 1910b, by original designation. Synonymy by Hood (1915).

Neoeurhynchothrips Watson, 1923. Type species Neoeurhynchothrips cubensis Watson, 1923, by monotypy. Synonymy by Stannard (1957).

Odontoplothrips Priesner, 1928d. Type species Trichothrips calcaratus Hood, 1925c, by monotypy. Synonymy by Moulton (1933b).

Hoplothrips (Chortothrips) Priesner, 1964. Type species not designated.
Maderothrips Priesner, 1964. Type species Trichothrips longisetis Bagnall, 1910c, by monotypy. Synonymy by Mound et al. (1976).

Bellicosothrips Johansen, 1981c. Type species Bellicosothrips magnificus Johansen, 1981c, by monotypy. Synonymy by Mound \& Marullo (1996).

Horistothrips Morgan, 1913 [5 species]. Type species Horistothrips australiae Morgan, 1913, by monotypy.

Aspidothrips Karny, 1920c. Type species Aspidothrips platygaster Karny, 1920c, by monotypy. Synonymy by Mound \& Houston (1987).

Hyidiothrips Hood, 1938d [10 species]. Type species Hyidiothrips atomarius Hood, 1938d, by monotypy.

Idiothrips Faure, 1933 [2 species]. Type species Idiothrips bellus Faure, 1933, by monotypy.

Iniothrips John, 1922 [1 species]. Type species Iniothrips procericornis John, 1922, by monotypy.

Iotatubothrips Mound \& Crespi, 1992 [2 species]. Type species Iotatubothrips crozieri Mound \& Crespi, 1992, by monotypy.

Isotrichothrips Priesner, 1968 [2 species]. Type species Liothrips longirostris Karny, 1912b, by original designation.

Jacobothrips zur Strassen, 1966 [1 1 species]. Type species Apterygothrips carolinae Faure, 1959, by monotypy.

Jacotia Faure, 1940 [5 species]. Type species Jacotia elegiae Faure, 1940, by monotypy.

Jennythrips Bhatti, 1993 [1 species]. Type species Liothrips (Zopyrothrips) jasmini Priesner, 1968, by monotypy.

Jersonithrips Retana \& Nishida, 2007 [1 species]. Type species Jersonithrips galligenus Retana \& Nishida, 2007, by monotypy.

Johansenthrips Retana-Salazar \& Soto- Rodríguez, 2008 [1 species]. Type species Johansenthrips galligena Retana-Salazar \& Soto- Rodríguez, 2008, by monotypy.

Karnyothrips Watson, 1923 [49 species]. Replacement name for Karynia Watson, 1922. Type species Karynia weigeli Watson, 1922 (=Anthothrips flavipes Jones, 1912), by monotypy.

Karynia Watson, 1922. Error for Karnyia Watson, homonym of Karnyia Shelford (Blattodea). 
Watsoniella Karny, 1923. Homonym of Watsoniella Berg, 1898 [Lepidoptera, Hesperidae] and a replacement name for Dolichothrips Watson, 1920, a homonym of Dolichothrips Karny, 1912. Type species Cephalothrips elongatus Watson, 1919 (=Karnyothrips melaleucus Bagnall), by monotypy.

Vargasia Retana-Salazar \& Soto-Rodríguez, 2007. Type species Karnyothrips melaleucus Bagnall, 1911 b, by monotypy. Synonymy by Goldarazena et al. (2008).

Dioclesianothrips Retana-Salazar \& Soto-Rodríguez, 2007. Type species Karnyothrips sonorensis Stannard, 1956 , by monotypy. Synonymy by Goldarazena et al. (2008)

Willeia Retana-Salazar \& Soto-Rodríguez, 2007. Type species Karnyothrips americanus Hood, 1912 d, by monotypy. Synonymy by Goldarazena et al. (2008).

Aguilaria Retana-Salazar \& Soto-Rodríguez, 2007. Nomen nudum. Synonymy by Goldarazena et al. (2008)

Katothrips Mound, 1971 [35 species]. Type species Kladothrips tytirus Girault, 1928b, by original designation.

Kellyia Bagnall, 1929f [13 species]. Type species Teuchothrips hoodianus Bagnall, 1924e, by monotypy.

Kladothrips Froggatt, 1906 [24 species]. Type species Kladothrips rugosus Froggatt, 1906, by monotypy.

Onychothrips Karny, 1911c. Type species Phloeothrips tepperi Uzel, 1905, by monotypy. Synonymy by Crespi et al. (2004).

Oncothrips Karny, 1911c. Type species Oncothrips tepperi Karny, 1911c, by monotypy. Synonymy by Crespi et al. (2004).

Brithothrips Moulton, 1942. Type species Brithothrips fuscus Moulton, 1942, by monotypy. Synonymy by Mound (1971).

Diplonychothrips Moulton, 1968. Type species Diplonychothrips antennatus Moulton, 1968, by monotypy. Synonymy by Mound (1971).

Klambothrips Mound \& Morris, 2007 [5 species]. Type species Klambothrips myopori Mound \& Morris, 2007, by original designation.

Kochummania Ananthakrishnan, 1969c [1 species]. Type species Kochummania excelsa Ananthakrishnan, 1969c, by monotypy.
Koptothrips Bagnall, 1929f [4 species]. Type species Koptothrips flavicornis Bagnall, 1929f, by monotypy.

Sphaericothrips Moulton, 1968. Type species Sphaericothrips clarapennis Moulton, 1968 (=Koptothrips flavicornis Bagnall), by monotypy. Synonymy by Mound (1971).

Kremnothrips Mound \& Tree, 2017 [1 species]. Type species Kremnothrips epakrus Mound \& Tree, 2017, by monotypy.

Leeuwenia Karny, 1912b [30 species]. Type species Leeuwenia gladiatrix Karny, 1912b, by monotypy.

Hoodiella Karny, 1923. Type species Leeuwenia convergens Hood, 1918, by monotypy. Synonymy by Mound (2004).

Hystricothripoides Fulmek, 1924. Type species Hystricothripoides karnyi Fulmek, 1924, by monotypy. Synonymy by Mound (2004).

Mametiella Priesner, 1949b. Type species Mametiella ardisiae Priesner, 1949b, by monotypy. Synonymy by Mound (2004).

Takahashia Ananthakrishnan, 1970a. Type species Leeuwenia pugnatrix Priesner, 1935c, by monotypy. Synonymy by Mound (2004).

Varshneyia Ananthakrishnan, 1973a. Replacement name for Takahashia Ananthakrishnan nec Takahashia Cockerell (Homoptera).

Leichhardtithrips Tree \& Mound, 2013 [1 species]. Type species Leichhardtithrips evanidus Tree \& Mound, 2013, by monotypy.

Leptoliothrips Moulton, 1927c [1 1 species $]$. Type species Leptoliothrips manilae Moulton, 1927c, by monotypy.

Leptothrips Hood, 1909 [30 species]. Type species Cryptothrips aspersus Hinds, 1902 (=Phloeothrips mali Fitch), by monotypy.

Lichanothrips Mound, 1971 [10 species]. Type species Lichanothrips albus Mound, 1971, by original designation.

Liophloeothrips Priesner, 1919a [18 species]. Type species Liophloeothrips glaber Priesner, 1919a, by monotypy.

Liothrips Uzel, 1895 [272 species]. Type species Phloeothrips setinodis Reuter, 1880 b, by subsequent designation of Hood (1918). 
Phyllothrips Hood, 1908b. Type species Phyllothrips citricornis Hood, 1908b, by original designation. Synonymy by Hood (1909).

Smerinthothrips Schmutz, 1909. Type species Smerinthothrips tropicus Schmutz, 1909, by monotypy. Synonymy by Priesner (1968).

Hoodia Karny, 1910. Type species Hoodia austriaca Karny, 1910, by monotypy. Synonymy by Priesner (1925d).

Rhynchothrips Hood, 1912c. Type species Rhynchothrips pruni Hood, 1912c, by original designation. Synonymy by Mound \& Marullo (1996).

Liothrips (Zopyrothrips) Priesner, 1968. Type species Gynaikothrips chavicae heptapleuri Karny, 1913c, by original designation. Synonymy by Priesner (1968).

Ataliothrips Bhatti, 1995c Type species Compsothrips reuteri Bagnall, 1913, by monotypy. Synonymy by Minaei \& Mound (2014).

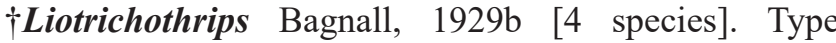
species Liotrichothrips hystrix Bagnall, 1929b, by monotypy.

Lispothrips Reuter, 1899 [6 species]. Type species Lispothrips wasastjernae Reuter, 1899, by monotypy.

Lissothrips Hood, 1908a [24 species]. Type species Lissothrips muscorum Hood, 1908a, by monotypy.

Trisclerothrips Stannard, 1953c. Type species Trisclerothrips hurricanus Stannard, 1953c, by monotypy. Synonymy by Mound (1989).

Litotetothrips Priesner, 1929b [10 10 species]. Type species Litotetothrips cinnamomi Priesner, 1929b (=Gynaikothrips rotundus Moulton), by monotypy.

Lizalothrips Okajima, 1984 [2 species]. Type species Lizalothrips luzonensis Okajima, 1984, by monotypy.

Logadothrips Priesner, 1929c [1 species]. Type species Logadothrips karnyellus Priesner, 1929c, by monotypy.

Lonchothrips Hood, 1957 [1 species]. Type species Lonchothrips linearis Hood, 1957, by monotypy.

Lygothrips Ananthakrishnan, 1964d [2 species]. Type species Eothrips jambuvasi Ramakrishna, 1928d, by monotypy.
Macrophthalmothrips Karny, 1922c [16 species]. Replacement name for Ophthalmothrips Karny nec Ophthalmothrips Hood, 1919a.

Ophthalmothrips Karny, 1920c. Type species Ophthalmothrips argus Karny, 1920c, by monotypy.

Majerthrips Mound \& Minaei, 2006 [1 species]. Type species Majerthrips barrowi Mound \& Minaei, 2006, by monotypy.

Malacothrips Hinds, 1902 [15 species]. Type species Malacothrips zonatus Hinds, 1902, by monotypy.

Udeothrips Hood, 1957. Type species Udeothrips vigilatus Hood, 1957, by monotypy. Synonymy by Mound (1977a).

Mallothrips Ramakrishna, 1928 [3 species]. Type species Mallothrips indicus Ramakrishna, 1928, by monotypy.

Manothrips Priesner, 1953 [1 species]. Type species Manothrips fortis Priesner, 1953, by monotypy.

Margaritothrips Priesner, 1932c [3 species]. Type species Margaritothrips sumatrensis Priesner, 1932c, by monotypy.

Mastigothrips Priesner, 1932c [2 species]. Type species Mastigothrips karnyianus Priesner, 1932c, by monotypy.

Mathetethrips Moulton, 1939 [1 species]. Type species Mathetethrips megacephalus Moulton, 1939, by monotypy.

Matilethrips Bournier \& Bournier, 1979 [1 species]. Type species Matilethrips inermis Bournier \& Bournier, 1979, by monotypy.

Maurithrips Mamet, 1967 [1 1 species]. Type species Maurithrips spinulosus Mamet, 1967, by monotypy.

Maxillata Faure, 1949b [3 species]. Type species Maxillata priesneri Faure, 1949b, by monotypy

Maxillithrips Bhatti, 1978f [1 species]. Type species Dolicholepta arorai Bhatti \& Hattar, 1974, by monotypy.

Medogothrips Han, 1988 [1 species]. Type species Medogothrips reticulatus Han, 1988, by monotypy.

Megeugynothrips Priesner, 1930a [1 species]. Type species Megeugynothrips efflatouni Priesner, 1930a, by monotypy.

Menothrips Hood, 1957 [2 species]. Type species Menothrips ebriosus Hood, 1957, by monotypy. 
Mesandrothrips Priesner, 1933c [21 species]. Type species Haplothrips inquilinus Priesner, 1921b, by monotypy.

Mesicothrips Priesner, 1952a [2 species]. Type species Mesicothrips plicans Priesner, 1952a, by monotypy.

Mesicothrips (Mesicothripoides) Ananthakrishnan, 1967. Type species Mesicothrips (Mesicothripoides) inquilinus Ananthakrishnan, 1967, by monotypy.

Mesothrips Zimmermann, 1900 [42 species]. Type species Mesothrips jordani Zimmermann, 1900, by subsequent designation of Priesner (1929b).

Trichaplothrips Priesner, 1921b. Type species Trichaplothrips sus Priesner, 1921b, by monotypy. Synonymy by zur Strassen (1994).

Mesothrips (Paramesothrips) Priesner, 1926c. Type species Neoheegeria mendax Karny, 1912b, by monotypy.

Metriothrips Hood, 1936b [6 species]. Type species Metriothrips midas Hood, 1936b, by monotypy.

Darwiniothrips Johansen, 1983a. Type species Darwiniothrips mayri Johansen, 1983a, by monotypy. Synonymy by Mound \& Marullo (1996).

Microdontothrips Okajima, 2006 [1 1 species]. Type species Microdontothrips argus Okajima, 2006, by monotypy.

Mimothrips Priesner, 1949b [3 species]. Type species Eurhynchothrips hargreavesi Priesner, 1935b, by original designation.

Mirothrips Cavalleri, Souza, Prezotto \& Mound, 2013 [4 species]. Type species Mirothrips bicolor Cavalleri, Souza, Prezotto \& Mound, 2013, by original designation.

Mixothrips Stannard, 1968b [2 species]. Type species Mixothrips craigheadi Stannard, 1968b, by monotypy.

Moultonides Kevin, 1963 [1 1 species]. Replacement name for Moultonia Bagnall nec Moultonia Bolivar (Orthoptera).

Moultonia Bagnall, 1928. Type species Dolerothrips geijerae Moulton, 1927a, by monotypy.

Murphythrips Mound \& Palmer, 1983b [1 species]. Type species Murphythrips legalis Mound \& Palmer, 1983 b, by monotypy.
Mutothrips Ananthakrishnan \& Swaminathan, 1980 [1 species]. Type species Mutothrips validus Ananthakrishnan \& Swaminathan, 1980, by monotypy.

Myopothrips Priesner, 1940a [1 species]. Type species Myopothrips symplocobius Priesner, 1940a, by monotypy.

Myrciathrips Cavalleri, Lindner \& Mendonça, 2016 [1 species]. Type species Myrciathrips variabilis Cavalleri, Lindner \& Mendonça, 2016, by monotypy.

Mystrothrips Priesner, 1949b [12 species]. Type species Sagenothrips dammermanni Priesner, 1933c, by monotypy.

Nagathrips Varatharajan \& Singh, 2000 [1 species]. Type species Mesothrips (Nagathrips) crenulatus Varatharajan \& Singh, 2000, by monotypy.

$\uparrow$ Necrothrips Priesner, 1924 [3 species]. Type species Necrothrips nanus Priesner, 1924, by monotypy.

Neocecidothrips Bagnall, 1929f [2 species]. Type species Eothrips bursariae Moulton, 1927a, by monotypy.

Neodixothrips Sen \& Muraleedharan, 1976 [1 species]. Type species Neodixothrips assamensis Sen \& Muraleedharan, 1976, by monotypy.

Neoheegeria Schmutz, 1909 [5 species]. Type species Neoheegeria dalmatica Schmutz, 1909, by monotypy.

Haplothrips (Gigaplothrips) Priesner, 1949b. Type species Haplothrips giganteus Priesner, 1934a, by monotypy. Synonymy by Minaei et al. (2007).

Neohoodiella Bournier, J.P., 1997 [2 species]. Type species Neohoodiella grandisetis Bournier, 1997, by monotypy.

Neothrips Hood, 1908a [3 species]. Type species Neothrips corticis Hood, 1908a, by monotypy.

Neurothrips Hood, 1924 [6 species]. Type species Acanthothrips magnafemoralis Hinds, 1902, by monotypy.

Ocnothrips Ananthakrishnan, 1969c [1 species]. Type species Ocnothrips indicus Ananthakrishnan, 1969c (=Austrothrips cochinchinensis Karny, 1922b), by monotypy.

Octurothrips Priesner, 1931c [1 species]. Type species Octurothrips pulcher Priesner, 1931c, by monotypy. 
Ocythrips Ananthakrishnan, 1972d [1 species]. Type species Ocythrips rarus Ananthakrishnan, 1972d, by monotypy.

Oidanothrips Moulton, 1944 [11 species]. Type species Oidanothrips magnus Moulton, 1944, by monotypy.

Okajimathrips Bhatti, 1992 [1 species]. Type species Podothrips kentingensis Okajima, 1986, by monotypy.

Opidnothrips Ananthakrishnan, 1971 [1 1 species]. Type species Opidnothrips corticulus Ananthakrishnan, 1971 , by monotypy.

Orthothrips Priesner, 1925b [10 species]. Type species Orthothrips caudatus Priesner, 1925b, by monotypy.

Apoxythrips Hood, 1957. Type species Apoxythrips stylifer Hood, 1957, by monotypy. Synonymy by Mound (1977a).

Ostlingothrips Crespi, Morris \& Mound, 2004 [2 species]. Type species Ostlingothrips pastus Crespi, Morris \& Mound, 2004, by original designation.

Pachyliothrips Okajima, 2006 [1 1 species $]$. Type species Pachyliothrips zelkovae Okajima, 2006, by monotypy.

Panceratothrips Bagnall, 1936 [1 1 species]. Type species Panceratothrips typicus Bagnall, 1936, by monotypy.

Panoplothrips Moulton, 1968 [1 species]. Type species Panoplothrips australiensis Moulton, 1968, by monotypy.

Parabaphothrips Moulton, 1949 [1 1 species]. Type species Parabaphothrips coffeae Moulton, 1949, by monotypy.

Paracholeothrips Moulton, 1968 [5 species]. Type species Paracholeothrips validus Moulton, 1968, by monotypy.

$†$ Parahoplothrips Schliephake, 2003 [1 species]. Type species Parahoplothrips nicolaji Schliephake, 2003, by monotypy.

Paramystrothrips Bournier, 1971a [4 species]. Type species Paramystrothrips leclanti Bournier, 1971a, by monotypy.

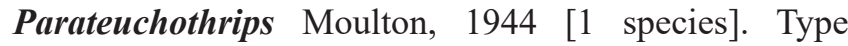
species Parateuchothrips fuscus Moulton, 1944, by monotypy.
Pedoeothrips Hood, 1954a [1 species]. Type species Pedoeothrips nigritus Hood, 1954a, by monotypy.

Pegothrips Sen \& Muraleedharan, 1976 [1 species]. Type species Pegothrips meghalaya Sen \& Muraleedharan, 1976 , by monotypy.

Pentagonothrips Haga \& Okajima, 1979a [1 species]. Type species Pentagonothrips antennalis Haga \& Okajima, 1979a, by monotypy.

Phallothrips Mound \& Crespi, 1992 [1 species]. Type species Phallothrips houstoni Mound \& Crespi, 1992, by monotypy.

Pharothrips Mound \& Wells, 2020 [1 species]. Type species Pharothrips hynnis Mound \& Wells, 2020, by monotypy.

Phasmothrips Priesner, 1933c [1 species]. Type species Phasmothrips asperatus Priesner, 1933c, by monotypy.

Phenicothrips Bhatti, 1995c [8 species]. Replacement name for Phaenothrips Priesner, nec Phenothrips Ananthakrishnan (1968a).

Phaenothrips Priesner, 1968a. Type species Gynaikothrips daetymon Karny, 1923, by original designation.

Phenothrips Ananthakrishnan, 1968a [1 species]. Type species Phenothrips decoratus Ananthakrishnan, 1968a, by monotypy.

Phiarothrips Ananthakrishnan, 1968b [1 species]. Type species Phiarothrips reperticus Ananthakrishnan, 1968 b, by monotypy.

Philothrips Priesner, 1939 [1 1 species]. Type species Philothrips socius Priesner, 1939, by monotypy.

Phlaeothrips Haliday, 1836 [24 species]. Type species Phlaeothrips coriacea Haliday, 1836, by subsequent designation in ICZN Opinion 865 (1969).

Phloeothrips Amyot \& Serville, 1843. Type species Phlaeothrips coriacea Haliday, 1836.

Phlaeothrips (Odontinothrips Priesner, 1914. Type species Phlaeothrips (Odontinothrips) denticauda Priesner, 1914, by monotypy.

Euphlaeothrips Morison, 1949. Type species Phlaeothrips coriaceus Haliday, 1836, by original designation.

Phorinothrips Ananthakrishnan, 1968a [3 species]. Type species Phorinothrips loranthi Ananthakrishnan, 1968a, by monotypy. 
Phylladothrips Priesner, 1933c [9 species]. Type species Phylladothrips karnyi Priesner, 1933c, by monotypy.

Paradexiothrips Okajima, 1984. Type species Paradexiothrips bispinosus Okajima, 1984, by monotypy. Synonymy by Okajima (1988).

Pistillothrips Johansen, 1982a [1 species]. Type species Pistillothrips guadalupae Johansen, 1982a, by monotypy.

Plagiothrips Priesner, 1968 [1 species]. Type species Gynaikothrips eugeniae Costa Lima, 1935, by monotypy.

Plectrothrips Hood, 1908a [32 species]. Type species Plectrothrips antennatus Hood, 1908a, by monotypy.

Hammatothrips Priesner, 1932c. Type species Hammatothrips crassiceps Priesner, 1932c, by monotypy. Synonymy by Okajima (1981).

Pleurothrips Hood, 1957 [1 1 species]. Type species Pleurothrips collaris Hood, 1957, by monotypy.

Plicothrips Bhatti, 1979d [2 species]. Type species Hindsiana apicalis Bagnall, 1915a, by original designation.

Pnigmothrips Priesner, 1953 [1 species]. Type species Pnigmothrips medanensis Priesner, 1953, by monotypy.

Podothrips Hood, 1913a [31 species]. Type species Podothrips semiflavus Hood, 1913a, by monotypy.

Kentronothrips Moulton, 1928a. Type species Kentronothrips hawaiiensis Moulton, 1928a, by monotypy. Synonymy by Girault (1932)

Melampodothrips Priesner, 1938a. Type species Podothrips (Melampodothrips) graminum Priesner, 1938a, by monotypy. Synonymy by Bhatti (1991).

Podothrips (Neopodothrips) Ananthakrishnan, 1965b. Type species Podothrips (Neopodothrips) distinctus Ananthakrishnan, 1965b, by monotypy.

Podothrips (Saucrothrips) Ananthakrishnan, 1968a. Type species Podothrips (Saucrothrips) scitulus Ananthakrishnan, 1968a, by monotypy.

Poecilothrips Uzel, 1895 [3 5 species]. Type species Poecilothrips albopictus Uzel, 1895, by monotypy.

Cephalothripoides Bagnall, 1927b. Type species Cephalothrips harrisoni Bagnall 1926e (=Poecilothrips albopictus Uzel, 1895), by monotypy. Synonymy by Mound (1968).
$†$ Polygonothrips Schliephake, 1999 [1 5 species]. Type species Polygonothrips apertosetosus Schliephake, 1999 , by monotypy.

Pongola zur Strassen, 1959 [1 species]. Type species Pongola rufianalis zur Strassen, 1959, by monotypy.

Ponticulothrips Haga \& Okajima, 1983 [1 species]. Type species Ponticulothrips diospyrosi Haga \& Okajima, 1983, by monotypy.

Porcothrips Priesner, 1951 [1 species]. Type species Terthrothrips liberiensis Priesner, 1936a, by monotypy.

Praeciputhrips Reyes, 1994 [1 species]. Type species Praeciputhrips balli Reyes, 1994, by monotypy.

Praepodothrips Priesner \& Seshadri, 1952 [7 species]. Type species Praepodothrips indicus Priesner \& Seshadri, 1952, by monotypy.

Preeriella Hood, 1939 [20 species]. Type species Chirothripoides minutus Watson, 1937b, by monotypy.

Machadonia Bournier, 1965. Type species Machadonia crassisetis Bournier, 1965, by original designation. Synonymy by Okajima (1998).

Priesneria Bagnall, 1926d [6 species]. Type species Priesneria kellyana Bagnall, 1926d, by monotypy.

Priesnerothrips Hood, 1954c [1 species]. Replacement name for Myopothrips Hood not Myopothrips Priesner, 1940a.

Myopothrips Hood, 1954a. Type species Myopothrips amazonicus Hood, 1954a, by monotypy.

Pristothrips Hood, 1925c [3 species]. Type species Pristothrips aaptus Hood, 1925c, by monotypy.

Celetothrips Morgan, 1929. Type species Celetothrips breviceps Morgan, 1929, by monotypy. Synonymy by Hood (1938c).

$†$ Prohaplothrips Schliephake, 2000 [1 species]. Type species Prohaplothrips iunctostylosus Schliephake, 2000, by monotypy.

$†$ Proleeuwenia Priesner, 1924 [1 species]. Type species Proleeuwenia succini Priesner, 1924, by monotypy.

Propealiothrips Reyes, 1994 [1 species]. Type species Propealiothrips moundi Reyes, 1994, by monotypy. 
Propesolomonthrips Reyes, 1994 [1 1 species]. Type species Propesolomonthrips mindorensis Reyes, 1994, by monotypy.

Prosantothrips Priesner, 1952a [1 species]. Type species Eothrips hyalopterus Karny, 1922b, by monotypy.

$†$ Protolispothrips Schliephake, 2001b [1 species]. Type species Protolispothrips multisetiger Schliephake, 2001b, by monotypy.

Psalidothrips Priesner, 1932c [48 species]. Type species Psalidothrips amens Priesner, 1932c, by monotypy.

Callothrips Ananthakrishnan, 1969b. Type species Callothrips ascitus Ananthakrishnan, 1969b, by monotypy. Synonymy by Okajima (1983).

Hennigthrips Johansen, 1986a. Type species Hennigthrips ananthakrishnani Johansen, 1986a, by monotypy. Synonymy by Mound \& Marullo (1996).

Pselaphothrips Hood, 1916a [2 species]. Type species Pselaphothrips pomeroyi Hood, 1916a, by monotypy.

Dinacanthothrips Priesner, 1932e. Type species Hoplandrothrips vuilleti Karny, 1920b, by monotypy. Synonymy by Pitkin \& Mound (1973).

Psenothrips Ananthakrishnan, 1968a [1 species]. Type species Teuchothrips priesneri Ananthakrishnan, $1964 c$, by monotypy.

Psephenothrips Reyes, 1994 [7 species]. Type species Psephenothrips strasseni Reyes, 1994, by monotypy.

Pseudophilothrips Johansen, 1979a [13 species]. Type species Pseudophilothrips moundi Johansen, 1979a, by monotypy.

Pueblothrips Stannard, 1950 [1 species]. Type species Pueblothrips minuta Stannard, 1950, by monotypy.

Pygmaeothrips Karny, 1920c [1 species]. Type species Pygmaeothrips columniceps Karny, 1920c (=Trichothrips angusticeps Hood, 1908), by monotypy.

Polyporothrips Watson, 1927. Type species Polyporothrips longipilosus Watson, 1927, by monotypy. Synonymy by Mound \& Marullo (1996).

Micropsothrips de Santis, 1950. Type species Micropsothrips ganodermae de Santis, 1950, by monotypy. Synonymy by Mound \& Marullo (1996)
Pyknothrips Ananthakrishnan, 1964b [1 species]. Type species Pyknothrips reticulatus Ananthakrishnan, $1964 b$, by monotypy.

Ramakrishnaiella Karny, 1926 [2 species]. Type species Ramakrishnaiella unispina Karny, 1926, by monotypy.

Retiothrips Bournier, 1994 [1 species]. Type species Retiothrips bambusae Bournier, 1994, by monotypy.

Rhaptothrips Crawford, 1909 [1 species]. Type species Rhaptothrips peculiaris Crawford, 1909, by monotypy.

Rhinoceps Faure, 1949a [3 species]. Type species Rhinoceps jansei Faure, 1949a, by monotypy.

Rhopalothripoides Bagnall, 1929e [6 species]. Type species Rhopalothrips brunneus Bagnall, 1916b, by original designation.

Froggattothrips Bagnall, 1929e. Type species Froggattothrips acaciae Bagnall, 1929e, by original designation. Synonymy by Mound (1968a).

Rosingothrips Reyes, 1994 [1 species]. Type species Rosingothrips ommatus Reyes, 1994, by monotypy.

Sacothrips Moulton, 1968 [7 species]. Type species Sacothrips bicolor Moulton, 1968, by monotypy.

Sagenothrips Priesner, 1933c [1 species]. Type species Sagenothrips gracilicornis Priesner, 1933c, by monotypy.

Sakimurella Bhatti, 1999b [1 species]. Replacement name for Sakimurathrips Bhatti 1995c nec Sakimurathrips Bhatti 1989 (=Merothripidae).

Sakimurathrips Bhatti, 1995c. Type species Liothrips kiriti Ramakrishna 1928, by monotypy.

Salothrips Ananthakrishnan, 1976a [1 species]. Type species Salothrips indicus Ananthakrishnan, 1976a, by monotypy.

Sartrithrips Mound \& Morris, 2001 [7 species]. Type species Sartrithrips popinator Mound \& Morris, 2001 , by original designation.

Scelothrips Priesner, 1952a [1 species]. Type species Scelothrips menoni Priesner, 1952a, by monotypy.

Schazothrips Hood, 1957 [1 species]. Type species Schazothrips anadenus Hood, 1957, by monotypy.

Schedothrips Ananthakrishnan, 1969c [2 species]. Type species Teuchothrips orientalis Ananthakrishnan, 1968a, by original designation. 
$†$ Schlechtendalia Bagnall, 1929b [1 1 species]. Type species Schlechtendalia longituba Bagnall, 1929b, by monotypy.

Schwarzithrips Morris \& Mound, 2000 [2 species]. Type species Schwarzithrips zammit Morris \& Mound, 2000 , by original designation.

Scopaeothrips Hood, 1912b [2 species]. Type species Scopaeothrips unicolor Hood, 1912b, by monotypy.

Rhopalothrips Hood, 1912b. Type species Rhopalothrips bicolor Hood, 1912b, by monotypy. Synonymy by Mound \& Marullo (1996).

Sedulothrips Bagnall, 1915c [2 species]. Type species Sedulothrips insolens Bagnall, 1915c, (=Polyommatothrips vigilans Hood, 1913b), by monotypy.

Senarioliothrips Johansen, 1981b [1979] [1 species]. Type species Senarioliothrips infrequentis Johansen, $1981 b$, by monotypy.

Senegathrips Bournier, 1971b [1 species]. Type species Senegathrips coutini Bournier, 1971b, by monotypy.

Senithrips Mound \& Minaei, 2006 [1 species]. Type species Senithrips psomus Mound \& Minaei, 2006, by monotypy.

Sinuothrips Collins, 2000 [1 species]. Type species Sinuothrips hasta Collins, 2000, by monotypy.

Smicrothrips Hood, 1952a [1 species]. Type species Smicrothrips particula Hood, 1952a, by monotypy.

Socothrips Ananthakrishnan, 1972c [1 species]. Type species Socothrips verrucosus Ananthakrishnan, 1972c, by monotypy.

Solomonthrips Mound, 1970a [9 species]. Type species Solomonthrips greensladei Mound, 1970a, by original designation.

Sophikothrips Mound, 1970a [1 1 species $]$. Type species Sophikothrips malaitae Mound, 1970a, by monotypy.

Sophiothrips Hood, 1934a [31 species]. Type species Sophiothrips squamosus Hood, 1934a, by original designation.

Nanothrips Faure, 1938. Type species Nanothrips breviceps Faure, 1938, by monotypy. Synonymy by Hood (1954d).

Bagnalliola Priesner, 1949a. Type species Brachythrips terminalis Bagnall, 1928a, by monotypy. Synonymy by Mound (1977c).
Zaxenothrips Crawford, 1943b. Type species Zaxenothrips peculiaris Crawford, 1943b, by monotypy. Synonymy by Hood (1954d).

Nanimothrips zur Strassen, 1974. Type species Nanimothrips makaronesicus zur Strassen, 1974, by monotypy. Synonymy by Mound (1977c).

Sphingothrips Ananthakrishnan, 1972a [1 species]. Type species Dolerothrips trachypogon Karny, 1923, by monotypy.

Amphidoxothrips Reyes, 1994. Type species Amphidoxothrips armatus Reyes, 1994, by monotypy. Synonymy by Bhatti (2004).

Spilothrips Moulton, 1942 [1 species]. Type species Spilothrips varicolor Moulton, 1942, by monotypy.

Stannardiana Ananthakrishnan, 1964b [1 species]. Type species Stannardiana variegata Ananthakrishnan, 1964b, by monotypy.

Stannardothrips Ananthakrishnan, 1965b [1 1 species]. Type species Stannardothrips longirostris Ananthakrishnan, 1965b, by monotypy.

Stegothrips Hood, 1934b [2 species]. Type species Stegothrips barronis Hood, 1934b, by monotypy.

Stenocephalothrips Johansen, 1982a [1 species]. Type species Stenocephalothrips zacualtipanensis Johansen, 1982a, by monotypy.

Stephanothrips Trybom, 1913 [32 species]. Type species Stephanothrips buffai Trybom, 1913, by monotypy.

Stictothrips Hood, 1925a [7 species]. Type species Phloeothrips maculatus Hood, 1909, by monotypy.

Stomothrips Okajima, 2000 [2 species]. Type species Stomothrips cycasi Okajima, 2000, by monotypy.

Strassenia Faure, 1959 [2 species]. Type species Strassenia acarus Faure, 1959, by monotypy.

Strepterothrips Hood, 1934a [15 species]. Type species Strepterothrips conradi Hood, 1934a, by monotypy.

Arcyothrips Hood, 1934b. Type species Arcyothrips africanus Hood, 1934b, by monotypy. Synonymy by Stannard (1957).

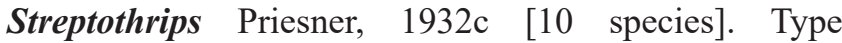
species Streptothrips mirabilis Priesner, 1932c, by monotypy.

Schizeia Faure, 1949a. Type species Schizeia chaberti Faure, 1949a, by original designation. Synonymy by Priesner (1949b). 
$†$ Sucinothrips Schliephake, 1999 [1 1 species]. Type species Sucinothrips incertus Schliephake, 1999, by monotypy.

Sumatrothrips Priesner, 1952a [ [ 11 species $]$. Type species Sumatrothrips filiceps Priesner, 1952a, by monotypy.

Sunaitiothrips Moulton, 1942 [1 species]. Type species Sunaitiothrips fuscus Moulton, 1942, by monotypy.

Suocerathrips Mound \& Marullo, 1994 [1 species]. Type species Suocerathrips linguis Mound \& Marullo, 1994, by monotypy.

Symphyothrips Hood \& Williams, 1915 [8 species]. Type species Symphyothrips punctatus Hood \& Williams, 1915, by monotypy.

Mesopotamothrips Liebermann \& Gemignani, 1931. Type species Mesopotamothrips concordiensis Liebermann \& Gemignani, 1931, by monotypy. Synonymy by de Santis (1959a).

Synergothrips Ananthakrishnan, 1972a [1 species]. Type species Synergothrips prolatus Ananthakrishnan, 1972 a, by monotypy.

Talitha Faure, 1958a [3 species]. Type species Talitha fusca Faure, 1958a, by original designation.

Tamilthrips Bhatti, 1995c [1 species]. Type species Apterygothrips pini Ananthakrishnan, 1960, by monotypy.

Temenothrips Okajima \& Urushihara, 1992 [3 species]. Type species Temenothrips flavillus Okajima \& Urushihara, 1992, by monotypy.

Terthrothrips Karny, 1925b [29 species]. Type species Phloeothrips sanguinolentus Bergroth, 1896, by monotypy.

Tetracanthothrips Bagnall, 1915b [1 species]. Type species Tetracanthothrips borneensis Bagnall, 1915 b, by monotypy.

Tetradothrips Priesner, 1952a [1 species]. Type species Eothrips foliiperda Karny, 1926, by monotypy.

Tetragonothrips Moulton, 1940 [1 species]. Type species Tetragonothrips murmekiai Moulton, 1940, by monotypy.

Teuchothrips Hood, 1919c [24 species]. Type species Teuchothrips simplicipennis Hood, 1919c, by original designation.
Thaumatothrips Karny, 1922a [1 1 species]. Type species Thaumatothrips froggatti Karny, 1922a, by monotypy.

Thilakothrips Ramakrishna, 1928 [1 1 species]. Type species Thilakothrips babuli Ramakrishna, 1928, by monotypy.

Thlibothrips Priesner, 1952a [7 species]. Type species Dolerothrips atavus Karny, 1915c, by original designation.

Thorybothrips Priesner, 1924c [2 species]. Type species Thorybothrips graminis Priesner, 1924c (=Cryptothrips unicolor Schille, 1911), by monotypy.

Tolmetothrips Priesner, 1953 [2 species]. Type species Eugynothrips smilacis Priesner, 1928b, by monotypy.

Torvothrips Johansen, 1979a [5 species]. Type species Torvothrips atrox Johansen, 1979a, by monotypy.

Trachythrips Hood, 1930 [11 species]. Type species Trachythrips watsoni Hood, 1930, by monotypy.

Tragothrips Pelikán, 2001 [1 1 species]. Type species Tragothrips kubani Pelikán, 2001, by monotypy.

Treherniella Watson, 1923 [6 species]. Type species Haplothrips orlando Watson \& Osborn, 1919, by monotypy.

Triadothrips Crespi, Morris \& Mound, 2004 [3 species]. Type species Triadothrips briga Crespi, Morris \& Mound, 2004, by original designation.

Trichinothrips Bagnall, 1929d [8 5 species]. Type species Trichinothrips branderi Bagnall, 1929d (=Trichaplothrips breviceps Bagnall, 1926d), by monotypy.

Tropothrips Hood, 1949 [5 species]. Type species Tropothrips borgmeieri Hood, 1949, by monotypy.

Truncatothrips Crespi, Morris \& Mound, 2004 [1 species]. Type species Truncatothrips terryae Crespi, Morris \& Mound, 2004, by monotypy.

Trybomia Karny, 1911b [6 species]. Type species Trybomia phasma Karny, 1911b (=Liothrips intermedius Bagnall), by monotypy.

Trypanothrips Hood, 1957 [1 species]. Type species Trypanothrips coxalis Hood, 1957, by monotypy.

Tumidothrips Pelikán, 2004 [1 species]. Type species Tumidothrips spiniceps Pelikán, 2004, by monotypy. 
Turmathrips Crespi, Morris \& Mound, 2004 [2 species]. Type species Turmathrips apistus Crespi, Morris \& Mound, 2004, by original designation.

Tylothrips Hood, 1937b [24 species]. Type species Tylothrips concolor Hood, 1937b, by monotypy.

Phragmothrips Hood, 1957. Type species Phragmothrips majusculus Hood, 1957, by monotypy. Synonymy by Mound (1977a).

Copiothrips Hood, 1957. Type species Copiothrips fuscifrons Hood, 1957, by monotypy. Synonymy by Mound (1977a).

Urothrips Bagnall, 1909d [12 species]. Type species Urothrips paradoxus Bagnall, 1909d, by monotypy.

Coxothrips Bournier, 1963. Type species Coxothrips reticulatus Bournier, 1963, by monotypy. Synonymy by Ulitzka \& Mound (2014).

Ananthakrishnaniella Stannard, 1970. Type species Ananthakrishnaniella tarai Stannard, 1970, by monotypy. Synonymy by Bhatti (1998b).

Biconothrips Stannard, 1970. Type species Biconothrips reedi Stannard, 1970, by monotypy. Synonymy by Ulitzka \& Mound (2014).

Veerabahuthrips Ramakrishna, 1932 [8 species]. Type species Veerabahuthrips bambusae Ramakrishna, 1932, by monotypy.

Mychiothrips Haga \& Okajima, 1979b. Type species: Mychiothrips fruticola Haga \& Okajima, 1979b, by monotypy. Synonymy by Dang et al. (2014).

Vicinothrips Mound \& Morris, 2000 [1 species]. Type species Vicinothrips bullatus Mound \& Morris, 2000, by monotypy.

Vuilletia Karny, 1923 [1 species]. Type species Trichothrips houardi Vuillet, 1914b, by monotypy.

Walkerthrips Bhatti, 1995c [1 species]. Type species Azaleothrips neatus Mound \& Walker, 1986, by monotypy.

Warithrips Mound, 1971 [5 5 species]. Type species Warithrips maelzeri Mound, 1971, by original designation.

$†$ Weitschatithrips Schliephake, 2003 [1 species]. Type species Weitschatithrips apithanus Schliephake, 2003, by monotypy.

Williamsiella Hood, 1925c [26 species]. Type species Williamsiella bicoloripes Hood, 1925c, by monotypy.
Prolissothrips Morgan, 1925. Type species Lissothrips (Prolissothrips) stratulus Morgan, 1925 (=Lissothrips breviceps Hood, 1925), by monotypy. Synonymy by Mound (1989).

Phthirothrips Priesner, 1933b. Type species Phthirothrips pediculus Priesner, 1933b, by monotypy. Synonymy by Mound (1989).

Microlissothrips Johansen \& Mojica, 1984. Type species Microlissothrips muscoaffinis Johansen \& Mojica, 1984, by monotypy. Synonymy by Mound (1989).

Xaniothrips Mound, 1971 [7 5 species]. Type species Xaniothrips xantes Mound, 1971, by original designation.

Xeroleptothrips Johansen, 1982b [1 species]. Type species Xeroleptothrips tehuacanensis Johansen, 1982b, by monotypy.

Xiphidothrips Priesner, 1951 [1 species]. Type species Xiphidothrips tambourissae Priesner, 1951, by monotypy.

Xyelethrips Mound, 1970c [1 species]. Type species Pygmaeothrips quadritibia Girault, 1927, by monotypy.

Xylaplothrips Priesner, 1928 [17 species]. Type species Cryptothrips fuliginosa Schille, 1911, by monotypy.

Xyloplothrips Bournier \& Bournier, 1986 [1 species]. Type species Xyloplothrips pelikani Bournier \& Bournier, 1986, by monotypy.

Yarnkothrips Mound \& Walker, 1986 [1 species]. Type species Yarnkothrips kolourus Mound \& Walker, 1986, by monotypy.

Zaliothrips Hood, 1938d [6 species]. Type species Zaliothrips citripes Hood, 1938d, by monotypy.

Zelotothrips Priesner, 1952a [1 species]. Type species Cryptothrips fuscipennis Karny, 1912b, by monotypy.

Zemiathrips Mound, 2002 [5 species]. Type species Zemiathrips triseta Mound, 2002, by original designation.

Zuluiella Jacot-Guillarmod, 1939 [1 1 species $]$. Type species Zuluiella distincta Jacot-Guillarmod, 1939, by monotypy. 


\section{References}

Alavi, J.A., Minaei, K. \& Fekrat, L. (2014) The Iranian Dendrothripinae (Thysanoptera: Thripidae) with description of a new genus and species. Zootaxa, 3860 (5), 479-486. https://doi.org/10.11646/zootaxa.3860.5.6

Amyot, C.J.B. \& Audinet-Serville, J.G. (1843) Histoire Naturelle des Insectes. Hemipteres. Paris.

Ananthakrishnan, T.N. (1956a) Erythrinothrips nov, - a new subgenus of Agnostothrips Moulton (Tubulifera, Thys.) from India. Indian Journal of Entomology, 17, 341-342.

Ananthakrishnan, T.N.(1956b) Studies on some Indian Thysanoptera III. Zoologischer Anzeiger, 157, 130-139.

Ananthakrishnan, T.N. (1957) Bamboosiella nov.gen. (Phlaeothripidae, Tubulifera) from India. Entomological News, 68, 65-68.

Ananthakrishnan, T.N. (1960) Thysanoptera from the Nilgiri and Kodaikanal Hills (South India). Journal of the Bombay Natural History Society, 57, 557-578.

Ananthakrishnan, T.N. (1961a) New Tubuliferan Thysanoptera from India. Journal of the Zoological Society of India, 12, $250-258$.

Ananthakrishnan, T.N.(1961b) Studies on some Indian Thysanoptera VI. Zoologischer Anzeiger, 167, 259-271.

Ananthakrishnan, T.N. (1962) Some little known Indian Terebrantia (Thysanoptera). Proceedings of the Royal Entomological Society of London (B), 31, 87-91. https://doi.org/10.1111/j.1365-3113.1962.tb01192.x

Ananthakrishnan, T.N. (1963) The Terebrantian Thysanoptera of the Indo-Ceylonese Region. Treubia, 26, 73-122.

Ananthakrishnan, T.N. (1964a) Indian Terebrantia - I. Bulletin of Entomology, Madras, 5, 49-55.

Ananthakrishnan, T.N. (1964b) Thysanopterologica Indica I. Entomologisk Tidskrift, 85, 99-120.

Ananthakrishnan, T.N. (1964c) Thysanopterologica Indica II. Entomologisk Tidskrift, 85, 218-235.

Ananthakrishnan, T.N. (1964d) A contribution to our knowledge of the Tubulifera (Thysanoptera) from India. Opuscula Entomologica Supplementum, 25, 1-120.

Ananthakrishnan, T.N. (1965a) Indian Terebrantia - II (Thysanoptera: Insecta). Bulletin of Entomology, Madras, 6, $15-29$.

Ananthakrishnan, T.N. (1965b) Thysanopterologica Indica - III Entomologisk Tidskrift, 86, 49-63.

Ananthakrishnan, T.N. (1966a) Thysanopterologica Indica - IV. Bulletin of Entomology, Madras, 7, 1-12.

Ananthakrishnan, T.N. (1966b) Indian Terebrantia - III. Bulletin of Entomology, Madras, 7, 26-33.

Ananthakrishnan, T.N. (1967) New Indian Thysanoptera. Annals de la Société entomologique Francais, 3, 227-237.

Ananthakrishnan, T.N. (1968a) Studies on new and little known Indian Thysanoptera. Oriental Insects, 1, 113-138. https://doi.org/10.1080/00305316.1967.10433856

Ananthakrishnan, T.N. (1968b) Thysanopterologica Indica - V. Oriental Insects, 2, 41-58.

https://doi.org/10.1080/00305316.1968.10433870

Ananthakrishnan, T.N. (1969a) On two new species of Terebrantian Thysanoptera with remarks on a new genus, Pellothrips. Oriental Insects, 2, 201-204.

https://doi.org/10.1080/00305316.1968.10433881
Ananthakrishnan, T.N. (1969b) Mycophagous Thysanoptera - 1 . Indian Forester, 95, 173-185.

Ananthakrishnan, T.N. (1969c) New gall thrips from India (Ins. Thysanoptera, Phlaeothripidae). Senckenbergiana biologica, 50, 179-194.

Ananthakrishnan, T.N. (1969d) Mycophagous Thysanoptera - II. Oriental Insects, 3, 289-299. https://doi.org/10.1080/00305316.1969.10433918

Ananthakrishnan, T.N. (1969e) Indian Thysanoptera. CSIR Zoological Monograph, 1, 1-171.

Ananthakrishnan, T.N. (1970a) Studies on the genus Leeuwenia Karny. Oriental Insects, 4, 47-58. https://doi.org/10.1080/00305316.1970.10433940

Ananthakrishnan, T.N. (1970b) On some species of Nesothrips Kirk., with notes on the influence of oedymerism and gynaecoidism in species of allied genera of Cryptothripini (Tubulifera; Thys.). Journal of the Zoological Society of India, 22, 51-62.

Ananthakrishnan, T.N. (1971) Mycophagous Thysanoptera - III. Oriental Insects, 5, 189-207. https://doi.org/10.1080/00305316.1971.10434008

Ananthakrishnan, T.N. (1972a) Further studies on Indian Gall Thrips. Marcellia, 37 (3), 111-127.

Ananthakrishnan, T.N. (1972b) Further studies on Indian Gall Thrips II. Marcellia, 37 (5), 3-20.

Ananthakrishnan, T.N. (1972c) Mycophagous Thysanoptera - IV. Oriental Insects, 6, 425-437. https://doi.org/10.1080/00305316.1972.10434186

Ananthakrishnan, T.N. (1972d) Mycophagous Thysanoptera - V. Oriental Insects, 6, 439-447. https://doi.org/10.1080/00305316.1972.10434187

Ananthakrishnan, T.N. (1973a) Two homonyms in Thysanoptera. Oriental Insects, 7, 48. https://doi.org/10.1080/00305316.1973.10434203

Ananthakrishnan, T.N. (1973b) Studies on some Indian species of the genus Elaphrothrips Buffa (Megathripinae: Tubulifera: Thysanoptera). Pacific Insects, 15, 271-284.

Ananthakrishnan, T.N. (1973c) Further studies on Indian Gall Thrips III. Oriental Insects, 7, 539-546. https://doi.org/10.1080/00305316.1973.10434426

Ananthakrishnan, T.N. (1976a) Two new genera and species of Tubuliferan thrips (Thysanoptera: Insecta) from India. Proceedings of the Indian Academy of Sciences (B), 83, 202206.

Ananthakrishnan, T.N. (1976b) New gall thrips of the genus Crotonothrips (Thysanoptera). Oriental Insects, 10, 411419. https://doi.org/10.1080/00305316.1976.10432341

Ananthakrishnan, T.N. \& Jagadish, A. (1967) Studies on the genus Chloethrips Priesner from India. Zoologischer Anzeiger, 178, 374-388.

Ananthakrishnan, T.N. \& Sen, S. (1980) Taxonomy of Indian Thysanoptera. Zoological Survey of India (Handbook Series), $1,1-234$.

Ananthakrishnan, T.N. \& Swaminathan, S. (1980) A new genus and two new species of thrips inhabiting Eugenia galls (Thysanoptera: Phlaeothripidae). Bulletin of the Zoological Survey of India, 2, 207-211.

Andre, F. (1940) The Nearctic species of Elaphrothrips Buffa (Thysanoptera: Phlaeothripidae). Proceedings of the 
Entomological Society of Washington, 42 (4), 75-90.

Back, E.A. (1912) Notes on Florida Thysanoptera, with description of a new genus. Entomological News, 23, 73-77.

Bagnall, R.S. (1908a) Notes on some genera and species new to the British fauna. Entomologist's monthly Magazine, 44, 3-7. https://doi.org/10.5962/bhl.part.1627

Bagnall, R.S. (1908b) On some new and curious Thysanoptera (Tubulifera) from Papua. Annals and Magazine of Natural History, (8) 1, 355-363.

https://doi.org/10.1080/00222930808692417

Bagnall, R.S. (1908c) On some new genera and species of Thysanoptera. Transactions of the Natural History Society of Northumberland, 3, 183-217.

Bagnall, R.S. (1909a) Synonymical notes; with a description of a new genus of Thysanoptera. Annals de la Société entomologique de Belge [1908], 52, 348-352.

Bagnall, R.S. (1909b) Preliminary description of a new and injurious Thrips. Entomologist's monthly Magazine, 45, 33-34. https://doi.org/10.5962/bhl.part.8930

Bagnall, R.S. (1909c) On the Thysanoptera of the Botanical Gardens, Brussels. Annals de la Société entomologique de Belge, 53, 171-176.

Bagnall, R.S. (1909d) On Urothrips paradoxus, a new type of Thysanopterous insects. Annales Historico-Naturales Musei Nationalis Hungarici, 7, 125-136.

Bagnall, R.S. (1909e) On some new and little known exotic Thysanoptera. Transactions of the Natural History Society of Northumberland, 3, 524-540.

Bagnall, R.S. (1909f) On two new genera of Thysanoptera from Venezuela; Anactinothrips and Actinothrips. Journal of the Linnean Society. Zoology, 30, 329-335.

https://doi.org/10.1111/j.1096-3642.1909.tb02139.x

Bagnall, R.S. (1910a) A contribution towards a knowledge of the neotropical Thysanoptera. Journal of the Linnean Society. Zoology, 30, 369-387. https://doi.org/10.1111/j.1096-3642.1910.tb02143.x

Bagnall, R.S. (1910b) Thysanoptera, pp. 669-701. In: Sharp, D. (ed.) Fauna hawaiiensis vol. 3. Cambridge University Press, London.

Bagnall, R.S. (1910c) On two new species of Trichothrips from the Derwent Valley. Transactions of the Natural History Society of Northumberland, 3, 661-663.

Bagnall, R.S. (1911a) Notes on some new and rare Thysanoptera (Terebrantia), with a preliminary list of the known British species. Journal of economic Biology, 6, 1-11.

Bagnall, R.S. (1911b) Descriptions of three new Scandinavian Thysanoptera (Tubulifera). Entomologist's monthly Magazine, 47, 60-63.

https://doi.org/10.5962/bhl.part.4953

Bagnall, R.S. (1912) On a new genus of Indian thrips (Thysanoptera) injurious to Turmeric. Records of the Indian Museum, 7, 257260.

https://doi.org/10.5962/bhl.part.28236

Bagnall, R.S. (1913a) Notes on Aeolothripidae, with description of a new species. Journal of economic Biology, 8, 155-158.

Bagnall, R.S. (1913b) Brief descriptions of new Thysanoptera. I. Annals and Magazine of Natural History, (8) 12, 290-299. https://doi.org/10.1080/00222931308693403

Bagnall, R.S. (1914a) Brief descriptions of new Thysanoptera. II. Annals and Magazine of Natural History, (8) 13, 22-31. https://doi.org/10.1080/00222931408693452

Bagnall, R.S. (1914b) Brief descriptions of new Thysanoptera. III. Annals and Magazine of Natural History, (8) 13, 287-297. https://doi.org/10.1080/00222931408693482

Bagnall, R.S. (1914c) Euthrips tamicola, a new species of Thysanoptera from the flowers of Black Bryony. Entomologist's monthly Magazine, 50, 273-274.

Bagnall, R.S. (1914d) Brief descriptions of new Thysanoptera. IV. Annals and Magazine of Natural History, (8) 14, 375-381. https://doi.org/10.1080/00222931408693588

Bagnall, R.S. (1914e) Fossil insect in amber. On Stenurothrips succineus gen. et sp. nov., an interesting Tertiary Thysanopteron. Geological Magazine, (6) 1, 483-485. https://doi.org/10.1017/S0016756800153294

Bagnall, R.S. (1915a) Brief descriptions of new Thysanoptera V. Annals and Magazine of Natural History, (8) 15, 315-324. https://doi.org/10.1080/00222931508693644

Bagnall, R.S. (1915b) Brief descriptions of new Thysanoptera VI. Annals and Magazine of Natural History, (8) 15, 588-597. https://doi.org/10.1080/00222931508693676

Bagnall, R.S. (1915c) On a collection of Thysanoptera from the West Indies, with descriptions of new genera and species. Journal of the Linnean Society. Zoology, 32, 495-507. https://doi.org/10.1111/j.1096-3642.1915.tb01871.x

Bagnall, R.S. (1916a) Brief descriptions of new Thysanoptera VII. Annals and Magazine of Natural History, (8) 17, 213-223. https://doi.org/10.1080/00222931508693771

Bagnall, R.S. (1916b) Brief descriptions of new Thysanoptera VIII. Annals and Magazine of Natural History, (8) 17, 397-412. https://doi.org/10.1080/00222931608693804

Bagnall, R.S. (1918) Brief descriptions of new Thysanoptera IX. Annals and Magazine of Natural History, (9) 1, 201-221. https://doi.org/10.1080/00222931808562303

Bagnall, R.S. (1919) Brief descriptions of new Thysanoptera X. Annals and Magazine of Natural History, (9) 4, 253-277. https://doi.org/10.1080/00222931908673889

Bagnall, R.S. (1921a) On Thysanoptera from the Seychelles Islands and Rodrigues. Annals and Magazine of Natural History, (9) 7, 257-293.

https://doi.org/10.1080/00222932108632523

Bagnall, R.S. (1921b) Brief descriptions of new Thysanoptera XI. Annals and Magazine of Natural History, (9) 7, 355-368. https://doi.org/10.1080/00222932108632530

Bagnall, R.S. (1921c) Brief descriptions of new Thysanoptera XII. Annals and Magazine of Natural History, (9) 8, 393-400. https://doi.org/10.1080/00222932108632598

Bagnall, R.S. (1923a) Fossil Thysanoptera I, Terebrantia Pt. I. Entomologist's monthly Magazine, 59, 35-38.

Bagnall, R.S. (1923b) Brief descriptions of new Thysanoptera XIII. Annals and Magazine of Natural History, (9) 12, 624-631. https://doi.org/10.1080/00222932308632986

Bagnall, R.S. (1924a) On a new injurious thrips affecting tea in India. Bulletin of Entomological Research, 14, 455. https://doi.org/10.1017/S0007485300045715

Bagnall, R.S. (1924b) On a new species of Rhipidothrips $(R$. kellyanus n. sp.) from Australia. Annals and Magazine of Natural History, (9) 13, 584-585. https://doi.org/10.1080/00222932308632986

Bagnall, R.S. (1924c) Von Schlechtendal's work on fossil Thysanoptera in the light of recent knowledge. Annals and 
Magazine of Natural History, (9) 14, 156-162. https://doi.org/10.1080/00222932408633102

Bagnall, R.S. (1924d) Fossil Thysanoptera III. Terebrantia Pt. 3. Entomologist's monthly Magazine, 60, 251-252.

Bagnall, R.S. (1924e) Brief descriptions of new Thysanoptera. XIV. Annals and Magazine of Natural History, (9) 14, 625-640. https://doi.org/10.1080/00222932408633174

Bagnall, R.S. (1926a) The family Franklinothripidae nov., with description of a new type of Thysanopteron. Annals and Magazine of Natural History, (9) 17, 168-173. https://doi.org/10.1080/00222932608633386

Bagnall, R.S. (1926b) Fossil Thysanoptera IV. Melanothripidae. Entomologist's monthly Magazine, 60, 16-17.

Bagnall, R.S. (1926c) Brief descriptions of new Thysanoptera XV. Annals and Magazine of Natural History, (9) 18, 98-114. https://doi.org/10.1080/00222932608633483

Bagnall, R.S. (1926d) Brief descriptions of new Thysanoptera XVI. Annals and Magazine of Natural History, (9) 18, 545-560. https://doi.org/10.1080/00222932608633551

Bagnall, R.S. (1926e) Contributions towards a knowledge of the European Thysanoptera I. Annals and Magazine of Natural History, (9) 18, 641-661.

https://doi.org/10.1080/00222932608633561

Bagnall, R.S. (1927a) Contributions towards a knowledge of the European Thysanoptera II. Annals and Magazine of Natural History, (9) 19, 564-575.

https://doi.org/10.1080/00222932708655536

Bagnall, R.S. (1927b) Contributions towards a knowledge of the European Thysanoptera III. Annals and Magazine of Natural History, (9) 20, 561-585.

https://doi.org/10.1080/00222932708655491

Bagnall, R.S. (1928a) Preliminary description of Mymarothrips ritchianus, a new type of Thysanopteron. Annals and Magazine of Natural History, (10) 1, 304-307. https://doi.org/10.1080/00222932808672783

Bagnall, R.S. (1928b) On some Samoan and Tongan Thysanoptera, with special reference to Ficus gall-causers and their inquilines. Thysanoptera. In Insects of Samoa, 7, 55-76. British Museum (Natural History), London.

Bagnall, R.S. (1929a) On the Australian Thysanoptera allied to the genus Odontothrips Uzel. Entomologist's monthly Magazine, $65,47-49$.

Bagnall, R.S. (1929b) Fossil Thysanoptera V. Tubulifera Pt. I. Entomologist's monthly Magazine, 65, 95-100.

Bagnall, R.S. (1929c) On some new and interesting Thysanoptera of economic importance. Bulletin of Entomological Research, 20, 69-76. https://doi.org/10.1017/S0007485300020940

Bagnall, R.S. (1929d) A new thrips-gall on Ironwood (Messua ferrea) and its inhabitants. Annals and Magazine of Natural History, (10) 3, 601-606.

https://doi.org/10.1080/00222932908673016

Bagnall, R.S. (1929e) On a group of minute Australian Thysanoptera (Tubulifera) and their association with the so-called leafglands of Acacia. Transactions of the entomological Society of London, 77, 171-176.

https://doi.org/10.1111/j.1365-2311.1929.tb00685.x

Bagnall, R.S. (1929f) On some new genera and species of Australian Thysanoptera (Tubulifera) with special reference to gallspecies. Marcellia, 25 [1928], 184-204.
Bagnall, R.S. (1929g) On Physothrips latus and P. propinquus Bagn. Entomologist's monthly Magazine, 65, 179-181.

Bagnall, R.S. (1931) On the Aeolothripid complex and the classification of the suborder Terebrantia (Thysanoptera). Bulletin de la Société des Naturalistes luxembourgeois, 7, 115-118.

Bagnall, R.S. (1932a) Descriptions of some new genera and species of African Aeolothripoid Thysanoptera. Annals and Magazine of Natural History, (10) 10, 287-294. https://doi.org/10.1080/00222933208673576

Bagnall, R.S. (1932b) Brief descriptions of new Thysanoptera. XVII. Annals and Magazine of Natural History, 10 (10), 505-520. https://doi.org/10.1080/00222933208673602

Bagnall, R.S. (1934) Contributions towards a knowledge of the European Thysanoptera. V. Annals and Magazine of Natural History, (10) 14, 481-500. https://doi.org/10.1080/00222933408654924

Bagnall, R.S. (1936) Descriptions of some new Thysanoptera from tropical Africa and Madagascar. Revue française d'entomologie, 3, 219-230.

Bagnall, R.S. \& Kelly, R. (1928) The genus Desmothrips Hood: with special reference to dimorphism in the sexes. Entomologist's monthly Magazine, 64, 204-206.

Beach, A.M. (1897) Contribution to a knowledge of the Thripidae of Iowa. Proceedings of the Iowa Academy of Sciences, 3, 214-227.

https://doi.org/10.5962/bhl.part.14877

Bergroth, E. (1896) Nouvelle especes de Thysanopteres. Annales de la Société Entomologique de Belgique, 40, 66-67. https://doi.org/10.5962/bhl.part.2022

Beshear, R.J. (1982) A new species of Frankliniella (Thysanoptera: Thripidae) from Georgia. Journal of the Georgia Entomological Society, 17, 72-75.

Bhatti, J.S. (1961) Anascirtothrips arorai novo genus and species, with notes on Chirothrips meridionalis Bagnall new to India. Bulletin of Entomology, Loyola College: Madras, 2, 26-29.

Bhatti, J.S. (1962)Anew genus and two new species of Thysanoptera, with notes on other species. Bulletin of Entomology, Loyola College: Madras, 3, 34-39.

Bhatti, J.S. (1963) Hyalopterothrips roonwali, a new thripid from India. Journal of the Zoological Society of India (1962), 14 (2), 176-178.

Bhatti, J.S. (1967a) Thysanoptera nova Indica. Published by the author, Delhi, 24 pp.

Bhatti, J.S. (1967b) New Indian Thysanoptera. 1. A new genus and two new species belonging to the tribe Sericothripini. Bulletin of Entomology, 8 (1), 59-64.

Bhatti, J.S. (1969a) Some synonyms chiefly among Indian Thysanoptera. Journal of the Bombay Natural History Society, 66 (1), 64-69.

Bhatti, J.S. (1969b) The taxonomic status of Megalurothrips Bagnall. Oriental Insects, 3 (3), 239-244. https://doi.org/10.1080/00305316.1969.10433912

Bhatti, J.S. (1970a) Taxonomic studies in some Thripini. Oriental Insects (1969), 3 (4), 373-381. https://doi.org/10.1080/00305316.1969.10433931

Bhatti, J.S. (1970b) A new genus Parsiothrips of the tribe Dendrothripini. Oriental Insects, 4 (2), 205-206. https://doi.org/10.1080/00305316.1970.10433956 
Bhatti, J.S. (1971a) Studies on some aeolothripids. Oriental Insects, 5 (1), 83-90. https://doi.org/10.1080/00305316.1971.10433992

Bhatti, J.S. (1971b) A new Chaetanaphothrips-like genus from South India, with a redefinition of Chaetanaphothrips. Oriental Insects, 5 (3), 337-343. https://doi.org/10.1080/00305316.1971.10434021

Bhatti, J.S. (1972) Three new species and two new genera of Indian Thripidae, with a record of two species new to India. Oriental Insects, 6 (4), 539-551. https://doi.org/10.1080/00305316.1972.10434194

Bhatti, J.S. (1973a) A preliminary revision of Sericothrips Haliday, sensu lat., and related genera, with a revised concept of the tribe Sericothripini. Oriental Insects, 7 (3), 403-449. https://doi.org/10.1080/00305316.1973.10434100

Bhatti, J.S. (1973b) A revision of the genus Caprithrips Faure. Oriental Insects, 7 (4), 475-484. https://doi.org/10.1080/00305316.1973.10434418

Bhatti, J.S. (1974) A new Anaphothripine genus from cane spindles in South India. Oriental Insects, 8 (4), 481-485. https://doi.org/10.1080/00305316.1974.10434880

Bhatti, J.S. (1976) Some new Indian Thripidae. Oriental Insects, $10(3), 317-326$. https://doi.org/10.1080/00305316.1976.10432331

Bhatti, J.S. (1978a) A revision of Karny's species of Anaphothrips of the Oriental region. Oriental Insects, 12 (1), 1-27. https://doi.org/10.1080/00305316.1978.10434547

Bhatti, J.S. (1978b) Systematics of Anaphothrips Uzel 1895 sensu latu and some related genera. Senckenbergiana biologica, 59 (1-2), 85-114.

Bhatti, J.S. (1978c) Preliminary revision of Taeniothrips. Oriental Insects, 12 (2), 157-199. https://doi.org/10.1080/00305316.1978.10434565

Bhatti, J.S. (1978d) Studies in the systematics of Rhamphothrips. Oriental Insects, 12 (3), 281-303. https://doi.org/10.1080/00305316.1978.10432090

Bhatti, J.S. (1978e) Four new species of Thysanoptera of India Oriental Insects, 12 (3), 419-432. https://doi.org/10.1080/00305316.1978.10432103

Bhatti, J.S. (1978f) A review of Dolichothrips Karny and Dolicholepta Priesner, with descriptions of two new genera. Entomon, 3 (2), 221-228.

Bhatti, J.S. (1979a) Eryngyothrips n. gen., with three species including a new one from Turkey. Senckenbergiana biologica, 59 (5-6), 389-397.

Bhatti, J.S. (1979b) A new genus of Thripidae from West African mosses with two new species. Senckenbergiana biologica, 60 (1-2), 75-84

Bhatti, J.S. (1979c) On two Thysanoptera (Insecta) of Nepal. Berichte des Naturwissenschaftlich-Medizinischen Vereins in Innsbruck, 66, 21-27.

Bhatti, J.S. (1979d) Studies in some haplothripine forms. Nouvelle Revue Entomologique, 9 (4), 305-311.

Bhatti, J.S. (1980) Species of the genus Thrips from India. Systematic Entomology, 5, 109-166. https://doi.org/10.1111/j.1365-3113.1980.tb00404.x

Bhatti, J.S. (1983) Revision of Thrips species described by Schmutz. Annalen des Naturhistorischen Museums in Wien, 84B, 479-507.

Bhatti, J.S. (1984) A remarkable Bregmatothrips-like new genus from Africa, with a review of Bregmatothrips Hood and Plutonothrips Priesner. Annals of Entomology, 2 (1), 83-97.

Bhatti, J.S. (1986) A remarkable new genus related with Thrips from North India. Zoology, 1 (1), 61-68.

Bhatti, J.S. (1988a) The spermatheca as a useful character for species differentiation in Coleothrips Haliday (Insecta: Terebrantia: Aeolothripidae). Zoology, 1 (2), 111-116. https://doi.org/10.46318/zoology.1.2.1988.111-116

Bhatti, J.S. (1988b) On the genera Ascirtothrips Priesner and Eremiothrips Priesner (Insecta: Terebrantia: Thripidae). Zoology, 1 (2), 117-125. https://doi.org/10.46318/zoology.1.2.1988.117-125

Bhatti, J.S. (1989) The classification of Thysanoptera into families. Zoology, 2 (1), 1-23. https://doi.org/10.46318/zoology.2.1.1989.1-23

Bhatti, J.S. (1990a) On some genera related to Chirothrips (Insecta: Terebrantia: Thripidae). Zoology, 2 (4), 193-200. https://doi.org/10.46318/zoology.2.4.1990.193-200

Bhatti, J.S. (1990b) Catalogue of insects of the Order Terebrantia from the Indian Subregion. Zoology, 2 (4), 205-352. https://doi.org/10.46318/zoology.2.4.1990.205-352

Bhatti, J.S. (1991) Surface patterns of wings in the Order Tubulifera (Insecta). New structural features and a new simple technique for microscopic surface scanning of the transparent wing membrane. Zoology, 3 (1), 1-95. https://doi.org/10.46318/zoology.3.1.1991.1-95

Bhatti, J.S. (1992) The Order Tubulifera (Insecta): Its characters and classification into families. Zoology, 3 (2), 127-162. https://doi.org/10.46318/zoology.3.2.1992.127-162

Bhatti, J.S. (1993) List of world genera of the Order Tubulifera (Insecta). Zoology, 3 (3), 241-271.

Bhatti, J.S. (1995a) Moundothrips, a new genus of Thripidae from Java, Indonesia (Insecta: Terebrantia). Zoology, 5 (1), 65-72. https://doi.org/10.46318/zoology.5.1.1995.73-95

Bhatti, J.S. (1995a) Further studies on Taeniothrips sensu lato (Insecta: Terebrantia: Thripidae). Zoology, 5 (1), 73-94. https://doi.org/10.46318/zoology.5.1.1995.73-95

Bhatti, J.S. (1995a) Studies on some Phlaeothripidae (Insecta: Tubulifera). Zoology, 5 (1), 97-110. https://doi.org/10.46318/zoology.5.1.1995.97-110

Bhatti, J.S. (1998a) The European and North American greenhouse pest Leucothrips nigripennis Reuter, the fern thrips (Terebrantia: Thripidae), lives outdoors in India. Oriental Insects, 32, 153-175. https://doi.org/10.1080/00305316.1998.10433772

Bhatti, J.S. (1998b) New structural features in the Order Tubulifera (Insecta). 2. Thoracic structures. Zoology, 5 (2), 177-252. https://doi.org/10.46318/zoology.5.2.1998.177-252

Bhatti, J.S. (1999a) Nomenclatural changes in Trichromothrips, Dorcadothrips, and Micothrips (Terebrantia: Thripidae). Thrips, 1, 1-5.

Bhatti, J.S. (1999b) Notes on Thysanoptera. Thrips, 1, 6-9. https://doi.org/10.46318/Thrips.1.1999.6-9

Bhatti, J.S. (1999c) The African genus Akheta of predatory thrips, with description of a new species from India (Terebrantia: Thripidae). Thrips, 1, 10-14. https://doi.org/10.46318/Thrips.1.1999.10-14

Bhatti, J.S. (2003a) The genera Tenothrips and Ewartithrips (Terebrantia: Thripidae) and pigmented facets of eye in some Terebrantia. Thysanoptera 2003, 1-10. 
https://doi.org/10.46318/Thrips.2.2003.1-10

Bhatti, J.S. (2003b) Species of Octothrips Moulton 1940 (Terebrantia: Thripidae) living on ferns in south and southeast Asia, with Apollothrips Wilson 1972 as new synonym. Thysanoptera, 2003, 15-24.

https://doi.org/10.46318/Thrips.2.2003.15-24

Bhatti, J.S. (2004) T.N. Ananthakrishnan's taxa of Thysanoptera 1950 to 1980 . Thysanoptera $2004,85-181$.

Bhatti, J.S. (2006) The classification of Terebrantia (Insecta) into families. Oriental Insects 40, 339-375. https://doi.org/10.1080/00305316.2006.10417487

Bhatti, J.S. \& de Borbón, C.M. (2008) A new genus and species of thrips related to Psilothrips Hood 1927 living on the South American saltbush, Atriplex lampa Gill. ex Moq. (Chenopodiaceae), in Argentina (Terebrantia: Thripidae). Thrips 6, 1-43. https://doi.org/10.46318/Thrips.6.2008.1-43

Bhatti, J.S. \& Mound, L.A. (1981) The genera of grass and cereal-feeding Thysanoptera related to the genus Thrips (Thysanoptera: Thripidae). Bulletin of Entomology, 21 (1980), 1-22.

Bhatti, J.S. \& Mound, L.A. (1992) A new genus of thripine Thysanoptera-Terebrantia with craspedote terga, from Trinidad. Zoology (Journal of Pure and Applied Zoology), 3 (2), 173-188.

Bhatti, J.S. \& Mound, L.A. (1994) A new genus of Terebrantian Thysanoptera from New Guinea, related to Foliothrips and Cestrothrips (Thripidae). Zoology (Journal of Pure and Applied Zoology), (1993), 4, 161-176.

Bhatti, J.S. \& Ranganath, H.R. (2006) A remarkable new thripid (Terebrantia: Thripidae) from jak trees (Moraceae) in Bangalore (Karnataka), India. Oriental Insects, 40, 379-380. https://doi.org/10.1080/00305316.2006.10417489

Bhatti, J.S., Veer, V \& Chauhan, N. (1994) Doonthrips setor, a new genus and species of Thripidae (Insecta: Terebrantia) from Dehradun (India). Zoology (1993), 4, 177-192.

Bhatti, J.S., Veer, V. \& Dahiya, A. (1999) The North American species Kurtomathrips morrilli Moulton and Neohydatothrips gracilipes (Hood) (Terebrantia: Thripidae) have now established large populations from North to South India. Thrips, 1, 54-57.

https://doi.org/10.46318/Thrips.1.1999.10-14

Bianchi, F.A. (1944) Nesothrips Kirkaldy supersedes Oedemothrips Bagnall. Proceedings of the Hawaiian entomological Society, $12,31-38$.

Bianchi, F.A. (1945) Introduction to the Thysanoptera of New Caledonia. Proceedings of the Hawaiian entomological Society, 12, 249-278.

Bianchi, F.A. (1946) Conocephalothrips tricolor, a new Urothripid from Hawaii. Proceedings of the Hawaiian entomological Society, 12, 499-502.

Bondar, G. (1924) Praga das roseiras na Bahia. Correio-Agricola Agricultura, Bahia, 2, 46-47.

Bondar, G. (1931) Un novo genero e tres novas especies de Thysanopteros Heliothripineos, encontrados na Bahia. Arquivos do Instituto Biologico, Sao Paulo, 4, 83-88.

Bouche, P.F. (1833) Naturgeschichte der schaldingen und nutzlichen Garten-Insekten und die bewahrtesten Mittel zur Vertilgung der ersteren. Berlin, pp. 1-176.

Bournier, A. (1962) Thysanoptères de France, II. Bulletin de la
Société entomologique de France, 67, 41-43.

Bournier, A. (1963) Thysanoptères de l'Angola II. Publicaçoes Culturais da Companhia de Diamantes de Angola, 63, 7386.

Bournier, A. (1965) Thysanoptères de l'Angola. III. Publicaçoes Culturais da Companhia de Diamantes de Angola, 72, 87106.

Bournier, A. (1967) Thysanoptères de Madagascar. Annales de la Société entomologique de France (N.S.), 3, 1015-1027.

Bournier, A. (1968) Thysanoptères de l'Angola IV. Publicaçoes Culturais da Companhia de Diamantes de Angola, 77, 133164.

Bournier, A. (1969a) Thysanoptères de France, IV. Annales de la Société entomologique de France (N.S.), 5, 329-360.

Bournier, A. (1969b) Thysanoptères de Madagascar, II. Bulletin de la Société zoologique de France, 94, 617-629.

Bournier, A. (1970) Thysanoptères du Gabon. Biologia Gabonica, $6,151-168$.

Bournier, A. (1971a) Thysanoptères de l'Angola, V. Publicaçoes Culturais da Companhia de Diamantes de Angola, 84, 6172.

Bournier, A. (1971b) Thysanoptères d'Afrique noire. Bulletin de l'Institut Fondamental de l'Afrique Noire, 33, 145-157.

Bournier, A. (1979) Thysanoptères d'Angola, VII. Garcia de Orta, Zoologia, 8, 1-10.

Bournier, A. (1985) Thysanoptères de Madagascar, IV. Nouvelle Revue d'Entomologie, 2, 331-334.

Bournier, A. (1990) Thysanoptères muscicoles de La Réunion, III. L'Entomologiste, 46, 301-310.

Bournier, A. (1993) Thysanoptères de Martinique et de Guadeloupe. Zoology (Journal of Pure and Applied Zoology), 3 (3), 227 240.

Bournier, A. (1994) Thysanoptères du Sénégal: espèces et genres nouveaux. Annales de la Société entomologique de France (N.S.), 30, 419-429.

Bournier, A. \& Bournier, J.-P. (1979) Thysanoptères d'Afrique noire, II. Bulletin de l'Institut Fondamental de l'Afrique Noire, 41, 355-365.

Bournier, A. \& Bournier, J.-P. (1986) Genre et espèce nouveaux du Languedoc (Thysanoptère-Phloeothripidae-Hoplothripini). Annales de la Société horticultural et Histoire naturelle Hérault, 3, 42-45.

Bournier, A. \& Bournier, J.-P. (1988) Nouvelles espèces muscicoles de La Reunion, II (Thysanoptera, Thripidae). Nouvelle Revue d'Entomologie, 5, 67-78.

Bournier, J.-P. (1997) Thysanoptères des forêts primaires de Nouvelle-Caledonie - I. Annals de la Societe entomologique de France, 33, 139-153.

Brethes, J. (1915) Descripcion de un Genero nuevo y una nueva especie de Thysanoptero de la Republica Argentina. Annales Museo Nacional, 27, 89-92.

Brothers, D.J. \& Mound, L.A. (1985) Eugynothrips Priesner, 1926 (Insecta, Thysanoptera): proposed designation of Cryptothrips conocephali Karny, 1963 as type species. Bulletin of Zoological Nomenclature, 42, 382-384.

https://doi.org/10.5962/bhl.part.963

Buffa, P. (1907) Alcune notizie sopra una nuova specie di tisanottero appartenente al genere Melanothrips Halid. Atti della Società Toscana di Scienze Naturali, 16, 58-61.

Buffa, P. (1908) Esame della raccolta di Tisanotteri italiani esistenti 
nel Museo Civico di Storia Naturale di Genova. Redia, 4, 382-391.

Buffa, P. (1909) I Tisanotteri esotici esistenti nel Museo Civico di Storia Naturale di Genova. Redia, 5, 157-172.

Burmeister, H. (1838) Handbuch der Entomologie, II (2), 404418.

Cavalleri, A., De Souza, A.R., Prezoto, F. \& Mound, L.A. (2013) Egg predation within the nests of social wasps: a new genus and species of Phlaeothripidae, and evolutionary consequences of Thysanoptera invasive behaviour. Biological Journal of the Linnean Society, 109, 332-341. https://doi.org/10.1111/bij.12057

Cavalleri, A., Lindner, M.F. \& Mendonça, Jr M. de S. (2016) New Neotropical Haplothripini (Thysanoptera: Phlaeothripidae) with a key to Central and South American genera. Journal of Natural History, 50, 1389-1410.

https://doi.org/10.1080/00222933.2015.1113316

Chen, L.-S. (1976) A new genus and species of Thripidae (Thysanoptera) from Taiwan. Bulletin of the Institute of Zoology, Academia Sinica, 14, 107-109.

Chen, L.-S. (1977) A new genus and two records of thrips (Thysanoptera: Terebrantia) from Taiwan. Plant Protection Bulletin (Taiwan), 18, 242-249.

Collins, D.W. (2000) A new genus and species of haplothripine thrips (Thysanoptera: Phlaeothripidae) with a prominent dorsal projection from the head. Insect Systematics and Evolution, 31, 285-289.

https://doi.org/10.1163/187631200X00048

Costa, A. (1888) Notizae ed osservazioni sulla geo-fauna Sarda. Atti della Reale Accademia delle Scienze Fisiche e Matematiche, ser. 2, 1 (2), 71.

Costa-Lima, A.M. (1935) Tisanopterocecidias do Brasil. O Campo, 6, 25-29.

Crawford, D.L. (1909) On some Thysanoptera from Mexico and the south. Pomona College Journal of Entomology, 1, 109-119.

Crawford, D.L. (1910) Thysanoptera of Mexico and the south II. Pomona College Journal of Entomology, 2, 153-170.

Crawford, J.C. (1938) A new genus and species of Thysanoptera from greenhouses. Proceedings of the Entomological Society of Washington, 40, 109-111.

Crawford, J.C. (1942) Two new South American species of Merothrips Hood (Thysanoptera: Merothripidae). Proceedings of the Entomological Society of Washington, 44, 150-154.

Crawford, J.C. (1943a) A new genus and species of Thysanoptera from New Zealand (Family Thripidae). Proceedings of the Entomological Society of Washington, 45, 151-153.

Crawford, J.C. (1943b) A new genus and species of Hoplothripini (Thysanoptera: Phlaeothripidae). Proceedings of the Entomological Society of Washington, 45, 221-225.

Crawford, J.C. (1945) A new genus and species of Thripinae from bulbs (Thysanoptera: Thripidae). Proceedings of the Entomological Society of Washington, 47, 92-94.

Crawford, J.C. (1948) A new genus of Urothripidae from Guatemala. Proceedings of the Entomological Society of Washington, 50, 39-40.

Crespi, B.J., Morris, D.C. \& Mound, L.A. (2004) Evolution of ecological and behavioural diversity: Australian Acacia thrips as model organisms. Australian Biological Resources Study, Canberra \& Australian National Insect Collection, Canberra. 328pp.
Dang, L.-H., Mound, L.A. \& Qiao, G.-X. (2013) Leaf-litter thrips of the genus Adraneothrips from Asia and Australia (Thysanoptera, Phlaeothripinae). Zootaxa, 3716 (1), 1-21. https://doi.org/10.11646/zootaxa.3716.1.1

Dang, L.-H., Mound, L.A. \& Qiao, G.-X. (2014) Conspectus of the Phlaeothripinae genera from China and Southeast Asia (Thysanoptera, Phlaeothripidae). Zootaxa, 3807 (1), 001082.

https://doi.org/10.11646/zootaxa.3807.1

Daniel, A.M. (1985) A new genus and species of fern infesting thrips (Thysanoptera: Insecta) with further notes on Mycterothrips nilgiriensis (Anan.). Current Science, 54, 528-530.

de Borbón, C.M. (2008) Desertathrips chuquiraga gen. et sp.n. (Thysanoptera, Thripidae) from Argentina. Zootaxa, 1751, 25-34.

https://doi.org/10.11646/zootaxa.1751.1.2

De Geer, C. (1773) Mémoires pour servir à l'histoire des insectes. Tome troisième. - pp. I-VIII [= 1-8], 1-696, [1-2], Pl. 1-44. Stockholm. (Hesselberg).

de Santis, L. (1950) Tres Tisanópteros nuevos de la República Argentina. Notas del Museo La Plata. Zoología, 15 (133), $59-70$.

de Santis, L. (1957) Adiciones a la fauna Argentina de Tisanópteros. Revista de la Facultad de Agronomía de La Plata, 33, 175182.

de Santis, L. (1959a) Notas sobre Tisanopteros Argentinos. Notas del Museo La Plata. Zoología, 19 (183), 247-259.

de Santis, L, (1959b) Adiciones a la fauna Argentina de Tisanópteros II. Acta Zoológica Lilloana, 17, 87-93.

de Santis, L. \& de Sureda, A.E.G. (1970) Un nuevo Heterothripido de Brasil (Thysanoptera). Studia Entomológica, 3, 471-473.

Dyadechko, N.P. (1961) A new thrips species Embothrips tubversicolor sp.nov. (Thysanoptera). Dopovidi Akademie Nauk Ukrayin'skoi RSR Kiev, 1961(5), 688-690.

Dyadechko, N.P. (1962) A new species of the genus Haplothrips (Thysanoptera) from the Crimea and Transcaucasus. Entomologicheskoe Obozrenie, 41, [1962], 160-162.

Dyadechko, N.P. (1964) Thrips (Thysanoptera) of the European part of the U.S.S.R., 387 pp. Kiev [in Russian].

Enderlein, G. (1929) Entomologica Canaria V. Die Thysanopteren der Retama-Blüte vom Pico de Teyde. Zoologischer Anzeiger, $86,39-44$.

Fabricius J.C. (1803) Systema Rhyngotorum secundum ordines, genera, species adjectis synonymis, locis, observationibus, descriptionibus. 314 pp. Brunsvigae.

https://doi.org/10.5962/bhl.title.11644

Faure, J.C. (1925) A new genus and five new species of South African Thysanoptera. South African Journal of Natural History, 5, 143-166.

Faure, J.C. (1933) New genera and species of Thysanoptera from South Africa. Bulletin of the Brooklyn Entomological Society, 28, 1-20; 55-75.

Faure, J.C. (1938) Descriptions of new Thysanoptera. Pretoria University Publications, Series 2, Natural Science, 4, 1-20.

Faure, J.C. (1940) Records and descriptions of South African Thysanoptera. I. Journal of the entomological Society of southern Africa, 3 159-172.

Faure, J.C. (1949a) Three remarkable new genera of Phlaeothripidae (Thysanoptera) from South Africa. Union of South Africa, Department of Agriculture, Entomology Memoirs, 2, 203- 
217.

Faure, J.C. (1949b) Maxillata priesneri gen. et spec. nov., a Thrips (Thysanoptera) with exceedingly long maxillae. Annals and Magazine of Natural History, (12) 2, 851-858. https://doi.org/10.1080/00222934908654029

Faure, J.C. (1953) Records and descriptions of South African Thysanoptera V. Journal of the entomological Society of southern Africa, 16, 194-216.

Faure, J.C. (1954) South African Thysanoptera - 1. Journal of the entomological Society of southern Africa, 17, 9-26.

Faure, J.C. (1956) Thysanoptera from papyrus in the Sudan. Journal of the entomological Society of southern Africa, 19, $100-117$.

Faure, J.C. (1957) South African Thysanoptera - 7. Journal of the entomological Society of southern Africa, 20, 391-419.

Faure, J.C. (1958a) South African Thysanoptera - 8. Journal of the entomological Society of southern Africa, 21, 16-35.

Faure, J.C. (1958b) South African Thysanoptera - 9. Journal of the entomological Society of southern Africa, 21, 354-375.

Faure, J.C. (1959) Thysanoptera of Africa - 1. Journal of the entomological Society of southern Africa, 22, 201-228.

Faure, J.C. (1961) Thysanoptera of Africa - 6. Revue de zoologie et de Botanique Africaine, 63, 66-95.

Field, H.H. (1910) Conspectus Methodicus. Bibliographica Zoologica, 17, 1-200.

Franklin, H.J. (1907) Ctenothrips, new genus. Entomological News, $1,247-250$

Franklin, H.J. (1908) On a collection of Thysanopterous insects from Barbados and St Vincent Islands. Proceedings of the United States National Museum, 33, 715-730. https://doi.org/10.5479/si.00963801.33-1590.715

Franklin, H.J. (1909) On Thysanoptera. Entomological News, 20, $228-231$.

Froggatt, W.W. (1906) Thrips or Black Fly (Thysanoptera). Agricultural Gazette of NSW, 17, 1005-1011.

Fulmek, L. (1924) Eine neue hystricothripide auf Eugenia sp. in Sumatra. Treubia, 6, 8-11.

Giard, A. (1901) Sur un thrips (Physopus rubrocincta nov. sp.) nuisible au Cacaoyer. Bulletin de la Societe Entomologique de France, 15, 263-265.

Girault, A.A. (1926) Characteristics of new Australian insects [Refused Publication on Pretext]. Published privately, Brisbane. 2pp.

Girault, A.A. (1927) Some new wild animals from Queensland. Published privately, Brisbane, 3 pp.

Girault, A.A. (1928a) A prodigeous discourse on wild animals. Published privately, Brisbane, 3 pp.

Girault, A.A. (1928b) Notice of a curious professor and of native wasps and woodlice. Published privately, Brisbane, $4 \mathrm{pp}$.

Girault, A.A. (1929) Description of a case of lunacy in Homo and of new six-legged articulates. Published privately, Brisbane, $4 \mathrm{pp}$.

Girault, A.A. (1930) New pests from Australia VII. Privately published, Brisbane, $3 \mathrm{pp}$.

Girault, A.A. (1932) New pests from Australia, X. Published privately, Brisbane, $6 \mathrm{pp}$.

Goldarazena, A. (2010) Bocathrips okajimai gen. et sp. n. (Thysanoptera, Phlaeothripidae), an Australian fungus-feeding thrips. Australian Journal of Entomology, 49, 121-125. https://doi.org/10.1111/j.1440-6055.2010.00750.x
Goldarazena, A. \& Infante, F. (2013) Guerothrips moundi, gen. et sp.n. (Thysanoptera, Thripidae) from Chiapas, Mexico. Zootaxa, 3669 (2), 172-178. https://doi.org/10.11646/Zootaxa.3669.2.9

Goldarazena, A., Mound, L.A. \& zur Strassen, R. (2008) Nomenclatural problems among Thysanoptera (Insecta) of Costa Rica. Revista Biologia Tropical, 56, 961-968. https://doi.org/10.15517/rbt.v56i2.5637

Gonzalez, C., Retana-Salazar, A.P. \& Castillo, N. (2010) Jessicathrips cubensis (Thysanoptera: Thripidae), nuevo género y especie para la ciencia. Revista de Protección Vegetal, 25 (2), 124-126.

Grimaldi, D., Shmakov, A. \& Fraser, N. (2004) Mesozoic thrips and early evolution of the order Thysanoptera (Insecta). Journal of Palaeontology, 78, 941-952.

https://doi.org/10.1666/0022-3360(2004)078<0941: MTAEEO $>2.0 . \mathrm{CO} ; 2$

Haga, K. \& Okajima, S. (1979a) A new glyptothripine genus and species (Thysanoptera, Phlaeothripidae) from Japan. Annotationes zoologicae Japonenses, 52, 146-150.

Haga, K. \& Okajima, S. (1979b) A new phlaeothripine genus and species (Thysanoptera, Phlaeothripidae) from Japan. Annotationes zoologicae Japonenses, 52, 266-271.

Haga, K. \& Okajima, S. (1983) A new genus and species of Phlaeothripidae (Thysanoptera) harmful to Persimmon from Japan. Annotationes zoologicae Japonenses, 56, 241-245.

Haliday, A.H. (1836) An epitome of the British genera in the Order Thysanoptera with indications of a few of the species. Entomological Magazine, 3, 439-451.

Haliday, A.H. (1852) Order III Physapoda. In: Walker F List of the Homopterous insects in the British Museum Part IV. London: British Museum, pp. 1094-1118.

Han, Y.F. (1988) Thysanoptera: Aeolothripidae, Thripidae, Phlaeothripidae. In: Huang, Fu-Sheng (Ed.) Insects of Mt. Namjagbarwa Region of Xizang. Science Press, Beijing, pp. 177-191.

Han, Y.F. (1991) A new genus and species of Sericothripina from China (Insecta: Thripidae). Acta Entomologica Sinica, 34, 208-211.

Han, Y.F. (1997) Thysanoptera: Aeolothripidae, Thripidae and Phlaeothripidae. pp. 531-571 in Insects of the Three Gorge Reservoir Area of Yangtze River.

Han, Y.F. \& Cui, Y.Q. (1992) Thysanoptera. pp. 420-434 in Insects of the Hengduan Mountains Region: Vol.1.

Hartwig, E.K. (1952) Taxonomic studies of South African Thysanoptera, including genitalia, statistics and a revision of Trybom's types. Entomology Memoirs, Department of Agriculture, South Africa, 2 (11), 341-469.

Heeger, E. (1852a) Beiträge zur Naturgeschichte der Physopoden (Blassenfusse). Sitzungsberichte der Kaiserlichen Akademie der Wissenschaften, Wien, 9, 123-141.

Heeger, E. (1852b) Beitrage zur Insecten-Fauna Osterrreichs. Sitzungsberichte der kaiserlichen Akademie der Wissenschaften, Wien, 9 (3), 473-490.

Heeger, E. (1854) Beiträge zur Naturgeschichte der Insecten Österreichs. Sitzungsberichte der kaiserlichen Akademie der Wissenschaften, Wien, 14 (3), 365-373.

Hinds, W.E. (1902) Contribution to a monograph of the insects of the order Thysanoptera inhabiting North America. Proceedings of the United States National Museum, 23, 79-242. 
https://doi.org/10.5479/si.00963801.26-1310.79

Hoddle, M.S, \& Mound, L.A. (2003) The genus Scirtothrips in Australia (Insecta, Thysanoptera, Thripidae). Zootaxa, 268, $1-40$.

https://doi.org/10.11646/zootaxa.268.1.1

Hood, J.D. (1908a) New genera and species of Illinois Thysanoptera. Bulletin of the Illinois State Laboratory of Natural History, 8 , 361-379.

https://doi.org/10.21900/j.inhs.v8.392

Hood, J.D. (1908b) Three new North American Phloeothripidae. Canadian Entomologist, 40, 305-309. https://doi.org/10.4039/Ent40305-9

Hood, J.D. (1909) A new genus and a new species of North American Phloeothripidae (Thysanoptera). Entomological News, 20, 249-252.

Hood, J.D. (1912a) Three new Phloeothripidae (Thysanoptera) from Texas and Michigan. Proceedings of the Biological Society of Washington, 25, 11-16.

Hood, J.D. (1912b) New genera and species of North American Thysanoptera from the South and West. Proceedings of the Biological Society of Washington, 25, 61-76.

Hood, J.D. (1912c) On North American Phloeothripidae (Thysanoptera), with descriptions of two new species. Canadian Entomologist, 44, 137-144. https://doi.org/10.4039/Ent44137-5

Hood, J.D. (1912d) A new genus and three new species of North American Thysanoptera. Psyche, 19, 113-118. https://doi.org/10.1155/1912/69610

Hood, J.D. (1912e) Descriptions of new North American Thysanoptera. Proceedings of the Entomological Society of Washington, 14, 129-160.

Hood, J.D. (1913a) Two new Thysanoptera from Porto Rico. Insecutor inscitiae menstruus, 1, 65-70. https://doi.org/10.5962/bhl.part.9573

Hood, J.D. (1913b) On a collection of Thysanoptera from Panama. Psyche, 20, 119-124. https://doi.org/10.1155/1913/48394

Hood, J.D. (1913c) A new species of Heliothrips (Thysanoptera) from Maryland and Illinois. Canadian Entomologist, 45, 308-311. https://doi.org/10.4039/Ent45308-9

Hood, J.D. (1914a) Two Porto Rican Thysanoptera from sugar cane. Insecutor inscitiae menstruus, 2, 38-41. https://doi.org/10.5962/bhl.part.9580

Hood, J.D. (1914b) On the proper generic names of certain Thysanoptera of economic importance. Proceedings of the Entomological Society of Washington, 16, 34-44.

Hood, J.D. (1914c) Studies in Tubuliferous Thysanoptera. Proceedings of the Biological Society of Washington, 27, 151-172.

Hood, J.D. (1915a) A remarkable new thrips from Australia. Proceedings of the Biological Society of Washington, 28, 49-51.

Hood, J.D. (1915b) An outline of the subfamilies and higher groups of the insect Order Thysanoptera. Proceedings of the Biological Society of Washington, 28, 53-60.

Hood, J.D. (1915c) Descriptions of new American Thysanoptera. Insecutor inscitiae menstruus, 3, 1-40.

Hood, J.D. (1915d) Hoplothrips corticis: a problem in nomenclature. The Entomologist, 48, 102-107.
Hood, J.D. (1915e) An interesting case of antennal antigeny in Thysanoptera. Proceedings of the Entomological Society of Washington, 17, 128-132.

Hood, J.D. (1916a) Two new Thysanoptera from West Africa, with a note on the synonymy of the Phloeothripidae. Psyche, 23, $6-12$. https://doi.org/10.1155/1916/48578

Hood, J.D. (1916b) Descriptions of new Thysanoptera. Proceedings of the Biological Society of Washington, 29, 109-124.

Hood, J.D. (1916c) Oedaleothrips hookeri, a new genus and species of Thysanoptera. Bulletin of the Brooklyn Entomological Society, 11, 64-65.

Hood, J.D. (1916d) A synopsis of the genus Oxythrips Uzel (Thysanoptera). Insecutor inscitiae menstruus, 4, 37-44.

Hood, J.D. (1918) New genera and species of Australian Thysanoptera. Memoirs of the Queensland Museum, 6, 121150.

Hood, J.D. (1919a) On some new Idolothripidae (Thysanoptera). Insecutor inscitiae menstruus, 7, 66-74.

Hood, J.D. (1919b) On some new Thysanoptera from southern India. Insecutor inscitiae menstruus, 7, 90-103.

Hood, J.D. (1919c) Two new genera and thirteen new species of Australian Thysanoptera. Proceedings of the Biological Society of Washington, 32, 75-92.

Hood, J.D. (1924) New Thysanoptera from the United States. Entomological News, 35, 312-317.

Hood, J.D. (1925a) On some new Phloeothripidae (Thysanoptera) from the Transvaal. Psyche, 31, 293-301. https://doi. org/10.1155/1924/29195

Hood, J.D. (1925b) Four new Thysanoptera from Africa. Proceedings of the Entomological Society of Washington, 27, $8-12$.

Hood, J.D. (1925c) New neotropical Thysanoptera collected by C.B. Williams. Psyche, 32, 48-69. https://doi.org/10.1155/1925/38498

Hood, J.D. (1925d) New species of Taeniothrips (Thysanoptera), from Africa. The Entomologist, 58, 132-140.

Hood, J.D. (1925e) Notes on New York Thysanoptera, with descriptions of new genera and species I. Bulletin of the Brooklyn Entomological Society, 20, 124-131.

Hood, J.D. (1925f) Four new Phloeothripidae from the United States (Thysanoptera). Canadian Entomologist, 57, 218-222. https://doi.org/10.4039/Ent57218-9

Hood, J.D. (1927a) A contribution toward the knowledge of New York Thysanoptera, with descriptions of new genera and species II. Entomologia Americana, 7, 209-245.

Hood, J.D. (1927b) New western Thysanoptera. Proceedings of the Biological Society of Washington, 40, 197-204.

Hood, J.D. (1928) New neotropical Thysanoptera collected by C.B. Williams II. Psyche, 34, 230-246. https://doi.org/10.1155/1927/87329

Hood, J.D. (1930) Two Urothripidae (Thysanoptera) from Florida, with keys to the known genera and the North American species. Bulletin of the Brooklyn Entomological Society, 24, 314-322.

Hood, J.D. (1931a) A new genus and species of Aeolothripidae (Thysanoptera) from Chile. Bulletin of the Brooklyn Entomological Society, 26, 1-3.

Hood, J.D. (1931b) Synonyms in the North American Thysanoptera. Pan-Pacific Entomologist, 7, 170-172. 
Hood, J.D. (1933a) Rhabdothrips albus, a new genus and species of Thysanoptera from Panama. Proceedings of the Entomological Society of Washington, 35, 45-48.

Hood, J.D. (1933b) Notothrips folsomi, a new genus and species of Thysanoptera from the United States. Proceedings of the Entomological Society of Washington, 35, 200-205.

Hood, J.D. (1934a) New Thysanoptera from Panama. Journal of the New York Entomological Society [1933], 41, 407-434.

Hood, J.D. (1934b) Two new genera and species of Phlaeothripidae (Thysanoptera). Proceedings of the Entomological Society of Washington, 36, 111-120.

Hood, J.D. (1935a) Some new or little known Thysanoptera of the family Phlaeothripidae. Revista de Entomologia , 5, 159-199.

Hood, J.D. (1935b) Eleven new Thripidae (Thysanoptera) from Panama. Journal of the New York Entomological Society, 43, 143-171.

Hood, J.D. (1936a) Nine new Thysanoptera from the United States. Journal of the New York Entomological Society, 44, 81-100.

Hood, J.D. (1936b) Studies in Neotropical Thysanoptera I. Revista de Entomologia, 6, 248-279.

Hood, J.D. (1936c) Studies in Neotropical Thysanoptera II. Revista de Entomologia, 6, 424-460.

Hood, J.D. (1937a) Studies on Neotropical Thysanoptera IV. Revista de Entomologia, 7, 255-296.

Hood, J.D. (1937b) Studies on Neotropical Thysanoptera V. Revista de Entomologia, 7, 486-530.

Hood, J.D. (1938a) A new subgenus and species of Thysanoptera from the Cameroons. Proceedings of the Biological Society of Washington, 51, 11-13.

Hood, J.D. (1938b) A new genus and species of Phlaeothripidae (Thysanoptera) from Palmetto. Proceedings of the Biological Society of Washington, 51, 27-32.

Hood, J.D. (1938c) Studies in Neotropical Thysanoptera VI. Revista de Entomologia, 8, 161-187.

Hood, J.D. (1938d) New Thysanoptera from Florida and North Carolina. Revista de Entomologia, 8, 348-420.

Hood, J.D. (1938e) Studies in Neotropical Thysanoptera VII. Revista de Entomologia, 9, 218-247.

Hood, J.D. (1938f) Studies in Neotropical Thysanoptera VIII. Revista de Entomologia, 9, 404-426.

Hood, J.D. (1939) New north American Thysanoptera, principally from Texas. Revista de Entomologia, 10, 550-619.

Hood, J.D. (1940) Organothrips bianchii, a new Hawaiian thrips from Taro. Proceedings of the Hawaiian entomological Society, 10, 423-427.

Hood, J.D. (1949) Brasilian Thysanoptera I. Revista de Entomologia, 20, 3-88.

Hood, J.D. (1950) Brasilian Thysanoptera II. Revista de Entomologia, 21, 1-113.

Hood, J.D. (1952) Brasilian Thysanoptera III. Proceedings of the Biological Society of Washington, 65, 141-174.

Hood, J.D. (1954a) Brasilian Thysanoptera IV. Proceedings of the Biological Society of Washington, 67, 17-54.

Hood, J.D. (1954b) A new Chirothrips-like genus of Thysanoptera from South Africa. Revue de Zoologie et Botanique Africaine, $49,1-5$.

Hood, J.D. (1954c) Brasilian Thysanoptera V. Proceedings of the Biological Society of Washington, 67, 195-214.

Hood, J.D. (1954d) New Thysanoptera, principally Floridian. Proceedings of the Biological Society of Washington, 67,
277-288

Hood, J.D. (1954e) Three new heliothripine Thysanoptera from Formosa. Proceedings of the Entomological Society of Washington, 56, 188-193.

Hood, J.D. (1954f) Angolan Thysanoptera I. A new genus and species of Heliothripinae. Publicaçóes Culturais da Companhia de Diamantes de Angola, 24, 23-31.

Hood, J.D. (1954g) Three new Thysanoptera from Trinidad and British Guiana. Proceedings of the Royal entomological Society of London B, 23, 205-212. https://doi.org/10.1111/j.1365-3113.1954.tb00093.x

Hood, J.D. (1956a) A new genus and species of Thripidae (Thysanoptera) from Trinidad. Proceedings of the Royal entomological Society of London B, 25, 64-66. https://doi.org/10.1111/j.1365-3113.1956.tb01091.x

Hood, J.D. (1956b) Two new Thripidae (Thysanoptera) from banana. Proceedings of the Biological Society of Washington, 69, 215-218.

Hood, J.D. (1957) New Brazilian Thysanoptera. Proceedings of the Biological Society of Washington, 70, 129-180.

Hood, J.D. \& Williams, C.B. (1915) New Thysanoptera from Florida and Louisiana. Journal of the New York Entomological Society, 23, 121-138.

International Code of Zoological Nomenclature (1999) [https:// www.iczn.org/the-code/the-international-code-of-zoologicalnomenclature/the-code-online/]

Jablonowski J (1894) Additamentum ad cognitionem Thysanopterorum. Termeszetrajzi Füzetek, 17, 93-99.

Jacot-Guillarmod, C.F. (1937) Ten new species of Thysanoptera and a catalogue of the known South African forms. Publications of the University of Pretoria (Series II, Natural Science), 3, $1-62$.

Jacot-Guillarmod, C.F. (1939) Phlaeothripidae (Thysanoptera) new to South Africa, with descriptions of new genera and species. Journal of the Entomological Society of Southern Africa, 2, $36-62$.

Jacot-Guillarmod, C.F. (1940) Studies on South African Thysanoptera - I. Journal of the Entomological Society of Southern Africa, 3, 131-138.

Jacot-Guillarmod, C.F. (1974) Catalogue of the Thysanoptera of the world (part 3). Annals of the Cape Provincial Museums Natural History, 7 (4), 517-976.

Jacot-Guillarmod, C.F. (1975) Catalogue of the Thysanoptera of the world (part 4). Annals of the Cape Provincial Museums Natural History, 7 (3), 977-1255.

Johansen, R.M. (1974) Siete nuevos Tisanopteros de Tabasco, Veracruz y el Pedregal de San Angel, Mexico, D.F. (Thysanoptera: Terebrantia; Tubulifera). Revista de la Sociedad Mexicana de Historia Natural, 35, 249-276.

Johansen, R.M. (1979a) Nuevos thrips tubuliferos (Insecta: Thysanoptera) de Mexico V. Anales del Instituto de Biologia de la Universidad Nacional Autónoma de Mexico 48 [1977], 77-92.

Johansen, R.M. (1979b) Notas sinonimicas acerca de Tisanopteros de Mexico. I. Anales del Instituto de Biologia de la Universidad Nacional Autónoma de Mexico, 49 [1978], 277-280.

Johansen, R.M. (1981a) Seis nuevos Tisanopteros (Terebrantia: Heterothripidae; Thripidae) de Chiapas, Mexico. Anales del Instituto de Biologia de la Universidad Nacional Autónoma de Mexico, 50 [1979], 159-178. 
Johansen, R.M. (1981b) Nuevos thrips tubuliferos (Insecta: Thysanoptera), de Mexico. VI. Anales del Instituto de Biologia de la Universidad Nacional Autónoma de Mexico, 50 [1979], 179-191.

Johansen, R.M. (1981c) Nuevos thrips tubuliferos (Insecta: Thysanoptera) de Mexico VII. Anales del Instituto de Biologia de la Universidad Nacional Autónoma de Mexico, 51, 337-346.

Johansen, R.M. (1982a) Nuevos thrips tubuliferos (Insecta: Thysanoptera) de Mexico IX. Anales del Instituto de Biologia de la Universidad Nacional Autónoma de Mexico, 52, 129150.

Johansen, R.M. (1982b) Nuevos thrips tubuliferos (Insecta: Thysanoptera) de Mexico X. Anales del Instituto de Biologia de la Universidad Nacional Autónoma de Mexico, 52, 151180.

Johansen, R.M. (1983a) Nuevos thrips tubuliferos (Insecta: Thysanoptera) de Mexico XI. Anales del Instituto de Biologia de la Universidad Nacional Autónoma de Mexico, 53, 5589.

Johansen, R.M. (1983b) Nuevos thrips (Insecta: Thysanoptera; Terebrantia, Thripidae, Thripinae) de La Sierra Madre Oriental y del eje Volcanico Transversal de Mexico. Anales del Instituto de Biologia de la Universidad Nacional Autónoma de Mexico Mexico, 53, 91-132.

Johansen, R.M. (1986a) Nuevos thrips tubuliferos (Insecta: Thysanoptera) de Mexico XIII. Anales del Instituto de Biologia de la Universidad Nacional Autónoma de Mexico, 56, 73-100.

Johansen, R.M. (1986b) Un genero nuevo Mexicano, afin al complejo generic Megalurothrips (Thysanoptera; Thripidae). Anales del Instituto de Biologia de la Universidad Nacional Autónoma de Mexico, 57, 93-111.

Johansen, R.M. (1997) Solanithrips n.gen. with two new species from Mexico living on Solanum (Insecta: Thysanoptera: Thripidae). Senckenbergiana biologica, 77, 107-116.

Johansen, R.M. \& Mojica, A.M. (1984) Nuevos thrips tubuliferos (Insecta: Thysanoptera) de Mexico XII. Anales del Instituto de Biologia de la Universidad Nacional de Mexico, 55, 55-94.

Johansen, R.M. \& Mojica, A.M. (1989) Una nueva especie Mexicana de Oxythrips Uzel, 1895 y un genero nuevo afin. Anales del Instituto de Biologia de la Universidad Nacional Autónoma de Mexico, 60, 199-204.

Johansen, R.M. \& Mojica-Guzman, A. (1996) A review of the Tribe Humboldthripini Johansen (Insecta, Thysanoptera: Thripidae). Folia entomologica Mexicana, 93 [1995], 39-70.

Johansen, R.M. \& Montes de Oca, L.M. (1989) Nuevos thrips tubuliferos (Insecta; Thysanoptera) de Mexico XIV. Anales del Instituto de Biologia de la Universidad Nacional Autónoma de Mexico, 60, 341-358.

John, O. (1921) A new genus and two new species of Thysanoptera from British East Africa. Annuaire du Musée zoologique de l'Académie des sciences de Russie, 23, 345-351.

John, O. (1924) Thysanopteren aus West-Sibirien. Entomologische Mitteilungen, 13, 7-10.

John, O. (1927) Une espece, une forme et une larve non decrites de Thysanopteres. Bulletin et Annales de la Société Entomologique de Belgique, 67, 205-209.

John, O. (1929) A new species of Thysanoptera from Brazil, representing a new genus. Bulletin et Annales de la Société
Entomologique de Belge, 69, 33-36.

Jones, P.R. (1912) Some new California and Georgia Thysanoptera. Technical series USDA Bureau of Entomology, 23, 1-24.

Karny, H. (1907) Die Orthopterenfauna des Küstengebietes von Österreich-Ungarn. Berliner Entomologische Zeitschrift, 52, $17-52$.

Karny, H. (1908) Die zoologische Reise des naturwissenschaftlichen Vereins nach Dalmatien im April 1906. Mitteilungen des Naturwissenschaftlichen Vereins an der Universität Wien, 6, 101-113.

Karny, H. (1910) Neue Thysanopteren der Wiener Gegend. Mitteilungen des Naturwissenschaftlichen Vereins an der Universität Wien, 8, 41-57.

Karny, H. (1911a) Revision der Gattung Heliothrips Haliday. Entomologische Rundschau, 28, 179-182.

Karny, H. (1911b) Neue Phloeothripiden-Genera. Zoologischer Anzeiger, 38, 501-504.

Karny, H. (1911c) Über Thrips-Gallen und Gallen-Thripse. Zentralblatt für Bakteriologie, Parasitenkunde, Infektionskrankheiten und Hygiene. Abteilung II, 30, 556572.

Karny, H. (1912a) Revision der von Serville aufgestellten Thysanopteren-Genera. Zoologische Annalen, 4, 322-344.

Karny, H. (1912b) Gallenbewohnende Thysanopteren aus Java. Marcellia, 1, 115-169.

Karny, H. (1912c) Zwei Neue javanische Physapoden-Genera. Zoologischer Anzeiger, 40, 297-301.

Karny, H. (1912d) Einige weitere Tubuliferen aus dem tropischen Afrika. Entomologische Rundschau, (20) 29, 130-133.

Karny, H. (1913a) Beitrag zur Thysanopteren-Fauna von NeuGuinea und Neu-Britannien. Archiv für Naturgeschichte, 79, $123-136$.

Karny, H. (1913b) H. Sauter's Formosa-Ausbeute. Supplementa Entomologica, 2, 127-134.

Karny, H. (1913c) Beiträge zur Kenntnis der Gallen von Java. 5. Über die javanischen Thysanoptero-cecidien und deren Bewohner. Bulletin de Jardin Botanique de Buitenzorg, 10, $1-126$.

Karny, H. (1913d) Thysanoptera. Wissenschaftliche Ergebnisse der Deutschen Zentral-Afrika Expedition 1907-1908, 4, 281282.

Karny, H. (1914a) Beitrag zur Thysanopterenfauna des Mediterrangebietes. Verhandlungen der zoologischbotanischen Gesellschaft in Wien, 64, 50-60.

Karny, H. (1914b) Beiträge zur Kenntnis der Gallen von Java. Zweite Mitteilung über die javanischen Thysanopterocecidien und deren Bewohner. Zeitschrift für wissenschaftliche Insektenbiologie, 10, 355-369.

Karny, H. (1915a) Beiträge zur Kenntnis der Gallen von Java. Zweite Mitteilung über die javanischen Thysanopterocecidien und deren Bewohner. Zeitschrift für wissenschaftliche Insektenbiologie, 11, 32-39.

Karny, H. (1915b) Beiträge zur Kenntnis der Gallen von Java. Zweite Mitteilung über die javanischen Thysanopterocecidien und deren Bewohner. Zeitschrift für wissenschaftliche Insektenbiologie, 11, 85-90.

Karny, H. (1915c) Beiträge zur Kenntnis der Gallen von Java. Zweite Mitteilung über die javanischen Thysanopterocecidien und deren Bewohner. Zeitschrift für wissenschaftliche 
Insektenbiologie, 11, 203-210.

Karny, H. (1915d) Beiträge zur Kenntnis der Gallen von Java. Zweite Mitteilung über die javanischen Thysanopterocecidien und deren Bewohner. Zeitschrift für wissenschaftliche Insektenbiologie, 11, 249-256.

Karny, H. (1916) Beiträge zur Kenntnis der Gallen von Java. Zweite Mitteilung über die javanischen Thysanopterocecidien und deren Bewohner. Zeitschrift für wissenschaftliche Insektenbiologie, 12, 15-22.

Karny, H. (1919) Synopsis der Megathripidae (Thysanoptera). Zeitschrift für wissenschaftliche Insektenbiologie, 1, 105-110 $+113-118$.

Karny, H. (1920a) Thysanopteren gesammelt von R. Ebner mit der Zoologischen Expedition nach dem Anglo-Ägyptischen Sudan, 1914. Akademie der Wissenschaften in Wien, Sitzung der mathematisch-naturwissenschaftlichen Klasse, 15, 2729.

Karny, H. (1920b) Die exotischen Tubuliferen (Thysanoptera) des Deutschen Entomologischen Museums (Berlin-Dahlem). Entomologische Mitteilungen, 9, 88-94 + 104-111 +186191.

Karny, H. (1920c) Nova Australska Thysanoptera, jez nashbiral Mjöberg. Casopisu Ceskoslovenské Spolecnosti Entomologiscké, 17, 35-44.

Karny, H. (1921a) Zur Systematik der orthopteroiden Insekten, Thysanoptera. Treubia, 1, 211-269.

Karny, H. (1921b) Beiträge zur malayischen Thysanopterenfauna. I. Eine neue Leeuwenia. II. Ein neuer Dinothrips. III. Über ein Merkwürdiges Vorkommen von Terebrantiern. Treubia, 1, 277-291.

Karny, H. (1921c) Beiträge zur malayischen Thysanopterenfauna IV. Thysanopteren von Hevea V. Thysanopteren an Tee. Treubia, 2, 37-83.

Karny, H. (1922a) A remarkable new gall-thrips from Australia. Proceedings of the Linnean Society of New South Wales, 47, 266-274.

Karny, H. (1922b) Thysanoptera from Siam and Indo-China. Journal of the Siam Society, 16, 91-153.

Karny, H. (1922c) Zorapteren aus Süd-Sumatra. Treubia, 3, 1437.

Karny, H. (1923) Beiträge zur Malayischen Thysanopterenfauna VI. Malayische Rindenthripse, gesammelt von Dr. NA Kemner. Treubia, 3, 277-380.

Karny, H. (1924) Results of Dr. E. Mjöberg's Swedish Scientific Expeditions to Australia 1910-1913. 38. Thysanoptera. Arkiv för Zoologi, 17A (2), 1-56.

Karny, H. (1925a) On some tropical Thysanoptera. Bulletin of entomological Research, 16, 125-142. https://doi.org/10.1017/S0007485300028431

Karny, H. (1925b) Über Phloeothrips sanguinolentus Bergroth nebst einer Revision der Diceratothripinen-Genera. Notulae entomologicae, 5, 77-84.

Karny, H. (1925c) Die an Tabak auf Java und Sumatra angetroffenen Blasenfüsser(Thysanoptera). Bulletin van het deli Proefstation te Medan, 23, 1-55.

Karny, H. (1926) Studies on Indian Thysanoptera. Memoirs of the Department of Agriculture in India. Entomology Series, 9, 187-239.

Kelly, R. \& Mayne, R.J.B. (1934) The Australian Thrips. A monograph of the Order Thysanoptera in Australia. Australian
Medical Publishing Co. Ltd, Glebe, New South Wales.

Kevin, D.K. (1963) A note on the Borneacridinae (Orth. Acridoidea). Eos (Madrid), 39, 279-283.

Kieffer, J.J. (1908) Description de galles et d'insectes gallicoles d'Asie. Marcellia, 7, 149-167.

Kirkaldy, G.W.(1907) On two Hawaiian Thysanoptera. Proceedings of the Hawaiian Entomological Society, 1 (3), 102-103.

Knechtel, W. (1923) Oxythrips dentatus nov. spec. eine neue Thysanopteren Spezies aus Rumänien. Bulletin de la Section Scientifique de l'Académie Roumaine, 8, 122-125.

Knechtel, W.(1960) Gen si specii noi de Thysanoptere. Comunicarile Academiei R. P. Romania, 10, 985-990.

Kobus, J.D. (1892) Blaaspooten (Thrips). Mededeelingen van het Proefstation Oost-Java, 43, 14-18.

Kono, T. \& O'Neill, K. (1964) The new generic status and synonymy of Anaphothrips arizonensis Morgan, with the description of the male (Thysanoptera, Thripidae). Bureau of Entomology, California Department of Agriculture, Occasional Papers, 6, $1-4$.

Kudô, I. (1974) Some graminivorous and gall-forming Thysanoptera of Taiwan. Kontyu, 42, 110-116.

Kudô, I. (1975) New name for the subgenus Telothrips Kudo \& Ananthakrishnan (Thysanoptera, Phlaeothripidae). Kontyu, 43, 421.

Kudô, I. (1977) A new genus and two new species of Thripidae (Thysanoptera) from Nepal. Kontyu, 45, 1-8.

Kudô, I. (1985) Yoshinothrips n. gen., with two new species from Japan (Thysanoptera: Thripidae). Kontyu, 53, 81-89.

Kudô, I. (1991) Sericothripine thrips of Japan (Thysanoptera, Thripidae). Japanese Journal of Entomology, 59, 509-538.

Kudô, I. (1992) A new Malaysian thrips with notes on some species of Dendrothripoides and Indusiothrips (Thysanoptera, Thripidae). Insecta matsumurana, 47, 91-101.

Kudô, I. \& Ananthakrishnan, T.N. (1974) A new subgenus and species of Meiothrips Priesner (Thys. Megathripinae) from Nepal. Kontyu, 42, 385-387.

Kumar, K., Tyagi, K. \& Bhatti, J.S. (2007) On the discovery of a new thrips related to the genus Haplothrips Amyot \& Serville from Delhi. Journal of the Bombay Natural History Society, 104, 68-71.

Kurosawa, M. (1954) Thrips collected by Dr. Hiroharu Yuasa with description of a new genus. Oyo-Kontyu, 10, 134-136.

Lacasa, A. (1983) Nuevo Tisanoptero de la Peninsula Iberica, Aeolothrips bournieri sp.n. (Aeolothripidae). Eos, 59, 67-75.

Li, Y.J., Li, Z.Y. \& Zhang, H.R. (2018) A new Panchaetothripinae genus and species, also a newly recorded genus, from Southwestern China (Thysanoptera: Thripidae). Zootaxa, 4394 (2), 235-242.

https://doi.org/10.11646/zootaxa.4394.2.6

Liebermann, J. \& Gemignani, E. (1931) Un nuevo genero y dos nuevas especies de Thysanopteros argentinos. Revista de la Sociedad Entomológia de Argentina, 3, 211-216.

Lima, E.F.B., O’Donnell, C.A. \& Miyasato, E.A. (2020) The Panchaetothripinae (Thysanoptera, Thripidae) of Brazil, with one new Caliothrips species. Zootaxa, 4820 (2), 201-230. https://doi.org/10.11646/zootaxa.4820.2.1

Linnaeus, C. (1758) Systema Naturae. Holmiae: Laurentii Salvii. 10th Edn pp. 1-823.

Liskiewicz, S. (1961) Turkmenothrips helenae n. subgen., n. sp., a new subgenus and species of Melanthrips Haliday 
(Thysanoptera, Aeolothripidae) from Turkmen SSR. Bulletin Entomologique de Pologne, 31 (23), 335-337.

Maltbaek, J. (1928) Thysanoptera Danica (Danske Frynsevinger). Entomologiske Meddelelser, 16, 159-184.

Mamet, J.R. (1967) Contribution to the knowledge of the Thysanoptera of Mauritius. Mauritius Institute Bulletin, 6, 64-88.

Marchal, P. (1910) Sur un nouveau «Thrips» vivant sur la vigne en Egypte. Bulletin de la Société Entomologique d'Égypte, $17-20$.

Martynov, A.V. (1935) A find of Thysanoptera in the Permian deposits. Comptes Rendus (Doklady) de l'Académie des Sciences de l'URSS, 3 (7), 333-336.

Marullo, R. \& Mound, L.A. (2001) Nexothrips; a new genus of thripine Thysanoptera (Insecta) from the neotropics, and its palaeotropical faunal affinities. Journal of the New York entomological Society, 108, 231-236.

https://doi.org/10.1664/0028-7199(2000)108[0231: NANGOT]2.0.CO;2

Masumoto, M., Ng, Y.-F. \& Okajima, S. (2013) A new genus of Thripinae (Thysanoptera, Thripidae) collected from Pandanus in Japan, Malaysia and Australia, with three new species. Zootaxa, 3709 (6), 543-554. https://doi.org/10.11646/zootaxa.3709.6.3

Masumoto, M. \& Okajima, S. (2003) Two new Dendrothripinae (Insecta, Thysanoptera, Thripidae) from Japan. Species Diversity, 8, 35-46.

https://doi.org/10.12782/specdiv.8.35

Masumoto, M. \& Okajima, S. (2005) A remarkable new genus of Thripinae (Thysanoptera, Thripidae) with enlarged metathoracic furca, from Southeast Asia. Zootaxa, 1048, 53-64.

https://doi.org/10.11646/zootaxa.1048.1.5

Masumoto, M. \& Okajima, S. (2007) The genus Scirtothrips Shull (Insecta, Thysanoptera, Thripidae) and three related genera in Japan. Zootaxa, 1552, 1-33 https://doi.org/10.11646/zootaxa.1552.1.1

Masumoto, M. \& Okajima, S. (2013) Review of the genus Thrips and related genera (Thysanoptera, Thripidae) from Japan. Zootaxa, 3678, 1-65. https://doi.org/10.11646/zootaxa.3678.1

Medina-Gaud, S. (1961) The Thysanoptera of Puerto Rico. Technical Paper of the University of Puerto Rico Agricultural Experimment Station, 32, 1-160.

Melis, A, (1931) Tisanotteri Italiani. Genus Melanthrips. Redia, 20, $1-143$.

Mirab-Balou, M., Mound, L.A. \& Tong, X.-L. (2015) New combinations and a new generic synonym in the genus Taeniothrips (Thysanoptera: Thripidae). Zootaxa, 3694 (3), 371-378.

https://doi.org/10.11646/zootaxa.3964.3.6

Minaei, K. (2012) Ficothrips, a new genus of Thripinae Thysanoptera from Iran. Zootaxa, 3361 (1), 63-68. https://doi.org/10.11646/zootaxa.3361.1.6

Minaei, K., Azemayeshfard, P. \& Mound, L.A. (2007) The southern Palaearctic genus Neoheegeria (Thysanoptera: Phlaeothripidae): redefinition and key to species. Tijdschrift voor Entomologie, 150, 55-64. https://doi.org/10.1163/22119434-900000212

Minaei, K. \& Mound, L.A. (2010) Grass-flower thrips of the genus
Chirothrips (Thysanoptera: Thripidae), with a key to species from Iran. Zootaxa, 2411, 33-43.

https://doi.org/10.11646/zootaxa.2411.1.3

Minaei, K. \& Mound, L.A. (2014) The Liothrips-lineage of thrips (Thysanoptera: Phlaeothripidae) from Iran with the first record of micropterous morph of a Liothrips species. Zootaxa, 3889 (1), 107-117.

https://doi.org/10.11646/zootaxa.3889.1.6

Morgan, A.C. (1913) New genera and species of Thysanoptera, with notes on distribution and food plants. Proceedings of the U.S. National Museum, 46, 1-55.

https://doi.org/10.5479/si.00963801.46-2008.1

Morgan, A.C. (1925) A new genus, a new subgenus and seven new species of Thysanoptera from Porto Rico. Florida Entomologist, 9, 1-7.

https://doi.org/10.2307/3492636

Morgan, A.C. (1929) A new genus and five new species of Thysanoptera foreign to the United States. Proceedings of the Entomological Society of Washington, 31, 1-9.

Morison, G.D. (1949) Thysanoptera of the London Area. London Naturalist Reprint 59, 1-131.

Morris, D.C., Mound, L.A. \& Schwarz, M.P. (2000) Advenathrips inquilinus: a new genus and species of social parasites (Thysanoptera: Phlaeothripidae). Australian Journal of Entomology, 39, 53-57. https://doi.org/10.1046/j.1440-6055.2000.00146.x

Moulton, D. (1911) Synopsis, catalogue and bibliography of North American Thysanoptera, with descriptions of new species. Technical Series, USDA Bureau of Entomology, 21, 1-56.

Moulton, D. (1926) New California Thysanoptera with notes on other species. Pan-Pacific Entomologist, 3, 19-28.

Moulton, D. (1927a) New gall-forming Thysanoptera of Australia. Proceedings of the Linnean Society of New South Wales, 52, 153-160.

Moulton, D. (1927b) Four new Californian Thysanoptera with notes on two other species. Pan-Pacific Entomologist, 4, 30-35.

Moulton, D. (1927c) Thysanoptera - New species and notes. Bulletin of the Brooklyn entomological Society, 22, 181-202.

Moulton, D. (1928a) Thysanoptera of the Hawaiian Islands. Proceedings of the Hawaiian entomological Society, 7, 105134.

Moulton, D. (1928b) Thysanoptera from Abyssinia. Annals and Magazine of Natural History, (10) 1, 227-248.

https://doi.org/10.1080/00222932808672872

Moulton, D. (1929a) Thysanoptera from India. Records of the Indian Museum, 31, 93-100.

Moulton, D. (1929b) New Thysanoptera from Cuba. Florida Entomologist, 13, 61-66. https://doi.org/10.2307/3492393

Moulton, D. (1929c) An interesting new thrips from Australia. Transactions of the Royal Society of South Australia, 53, 264-266.

Moulton, D. (1930a) Thysanoptera from Africa. Annals and Magazine of Natural History, (10) 5, 194-207. https://doi.org/10.1080/00222933008673119

Moulton, D. (1930b) A new genus and species of Thysanoptera from Chile with notes on other species. Revista Chilena Historia Naturale, 32, 272-275.

Moulton, D. (1931) An interesting new California thrips. PanPacific Entomologist, 7, 173-174. 
Moulton, D. (1932) The Thysanoptera of South America I. Revista de Entomologia, 2, 451-484.

Moulton, D. (1933a) The Thysanoptera of South America II. Revista de Entomologia, 3, 96-133.

Moulton, D. (1933b) The Thysanoptera of South America III. Revista de Entomologia, 3, 227-262.

Moulton, D. (1933c) Oligothrips oreios a new genus and species of thrips belonging to the family Opadothripidae Bagnall. PanPacific Entomologist, 9, 139-140.

Moulton, D. (1933d) The Thysanoptera of South America IV. Revista de Entomologia, 3, 385-419.

Moulton, D. (1934) New Thysanoptera of the Hawaiian Islands. Proceedings of the Hawaiian Entomological Society, 8, 499503.

Moulton, D. (1935a) In: Newman, L.J. Thrips census. New species of thrips from south-western Australia. Journal of the Royal Society of Western Australia, 21, 97-100.

Moulton, D. (1935b) A new thrips on cotton. Philippine Journal of Agriculture, 6. 475-477.

Moulton, D. (1939) Thysanoptera collected by the Mangarevan expedition. Occasional Papers of the Bishop Museum, 15, 141-148.

Moulton, D. (1940) Thysanoptera from New Guinea and New Britain. Occasional Papers of the Bishop Museum, 15, 243270

Moulton, D. (1941) Thysanoptera from Minas Geraes, Brazil (second paper). Revista de Entomologia, 12, 314-322.

Moulton, D. (1942) Seven new genera of Thysanoptera from Australia and New Zealand. Bulletin of the Southern California Academy of Science, 41, 1-13.

Moulton, D. (1944) Thysanoptera of Fiji. Occasional Papers of the Bishop Museum, 17, 267-311.

Moulton, D. (1947) Thysanoptera from New Guinea, the Philippine Islands and the Malay Peninsula. Pan-Pacific Entomologist, 23, 172-180.

Moulton, D. (1949) New Thysanoptera from Africa. Annals and Magazine of Natural History, (2) 12, 481-498. https://doi.org/10.1080/00222934908654000

Moulton, D. (1968) [published posthumously]. New Thysanoptera from Australia. Proceedings of the California Academy of Sciences 4th series, 36, 93-124.

Mound, L.A. (1967a) A new genus and species of Thysanoptera predatory on mites in Iraq. Bulletin of Entomological Research, 57, 315-319. https://doi.org/10.1017/S0007485300050021

Mound, L.A. (1967b) A taxonomic revision of the Australian Aeolothripidae (Thysanoptera). Bulletin of the British Museum (Natural History). Entomology, 20, 41-74.

Mound, L.A. (1968a) A review of R.S. Bagnall's Thysanoptera collections. Bulletin of the British Museum (Natural History). Entomology Supplement, 11, 1-181. [https://www. biodiversitylibrary.org/page/40874054]

Mound, L.A. (1968b) New synonymy in the South African genus Synaptothrips Trybom (Thysanoptera) with a key to the species. Journal of the Entomological Society of South Africa, 31, 87-95.

Mound, L.A. (1969) Revision of three Australian genera of Phlaeothripidae (Thysanoptera) with seven new species, and one new generic synonym. Journal of the Australian Entomological Society, 8, 173-186. https://doi.org/10.1111/j.1440-6055.1969.tb00754.x

Mound, L.A. (1970a) Thysanoptera from the Solomon Islands. Bulletin of the British Museum (Natural History). Entomology, 24, 83-126.

https://doi.org/10.5962/bhl.part.1519

Mound, L.A. (1970b) Studies on heliothripine Thysanoptera. Proceedings of the Royal Entomological Society of London (B), 39, 41-56. https://doi.org/10.1111/j.1365-3113.1970.tb00256.x

Mound, L.A. (1970c) Convoluted maxillary stylets and the systematics of some Phlaeothripine Thysanoptera from Casuarina trees in Australia. Australian Journal of Zoology, 18, 439-463. https://doi.org/10.1071/ZO9700439

Mound, L.A. (1971) Gall-forming thrips and allied species (Thysanoptera: Phlaeothripinae) from Acacia trees in Australia. Bulletin of the British Museum (Natural History). Entomology, 25, 387-466. https://doi.org/10.5962/bhl.part.19680

Mound, L.A. (1972a) Further studies on Australian Aeolothripidae (Thysanoptera). Journal of the Australian Entomological Society, 11, 37-54. https://doi.org/10.1111/j.1440-6055.1972.tb01603.x

Mound, L.A. (1972b) Species complexes and the generic classification of leaf-litter thrips of the Tribe Urothripini (Phlaeothripidae). Australian Journal of Zoology, 20, 83103. https://doi.org/10.1071/ZO9720083

Mound, L.A. (1974a) Spore-feeding Thrips (Phlaeothripidae) from Leaf Litter and Dead Wood in Australia. Australian Journal of Zoology. Supplement, 27, 1-106. https://doi.org/10.1071/AJZS027

Mound, L.A. (1974b) Andrethrips floydi - a remarkable new Thysanopteron. Journal of Entomology (B), 43, 109-113. https://doi.org/10.1111/j.1365-3113.1974.tb00095.x

Mound, L.A. (1974c) The Nesothrips complex of spore-feeding Thysanoptera (Phlaeothripidae: Idolothripinae). Bulletin of the British Museum (Natural History). Entomology, 31, 109188. https://doi.org/10.5962/bhl.part.29485

Mound, L.A. (1977a) Species diversity and the systematics of some New World leaf-litter Thysanoptera (Phlaeothripinae; Glyptothripini). Systematic Entomology, 2, 225-244. https://doi.org/10.1111/j.1365-3113.1977.tb00371.x

Mound, L.A. (1977b) Anew genus of Aeolothripidae (Thysanoptera) from New Zealand and New Caledonia. New Zealand Journal of Zoology, 4, 149-152. https://doi.org/10.1080/03014223.1977.9517946

Mound, L.A. (1977c) Leaf-litter Thysanoptera of the sub-tribe Williamsiellina (Phlaeothripidae). Bulletin of the British Museum (Natural History). Entomology, 36, 171-192. [https://www.biodiversitylibrary.org/page/2307805]

Mound, L.A. (1989) Systematics of thrips (Insecta: Thysanoptera) associated with mosses. Zoological Journal of the Linnean Society, 96, 1-17.

https://doi.org/10.1111/j.1096-3642.1989.tb01818.x

Mound, L.A. (1990) A new genus and species of Thripidae (Thysanoptera) from Western Australia with elongate mouth parts. Entomologist's Monthly Magazine, 126, 213-216.

Mound, L.A. (1991) The first thrips species (Insecta, Thysanoptera) 
from cycad male cones, and its family level significance. Journal of Natural History, 25, 647-652. https://doi.org/10.1080/00222939100770411

Mound, L.A. (1993) The first thrips species (Insecta) inhabiting leaf domatia: Domatiathrips cunninghamii gen. et sp. n. Journal of the New York Entomological Society, 101, 424-430. [https:// www.biodiversitylibrary.org/page/52158101]

Mound,L.A.(1995)Homoplasy and the systematics of phlaeothripine Thysanoptera, with a new short-tubed Australian Urothripine. Courier Forschunginstitut Senckenberg, 178, 21-25.

Mound, L.A. (1999) Saltatorial leaf-feeding Thysanoptera (Thripidae, Dendrothripinae) in Australia and New Caledonia, with newly recorded pests of ferns, figs and mulberries. Australian Journal of Entomology, 38, 257-273. https://doi.org/10.1046/j.1440-6055.1999.00112.x

Mound, L.A. (2002) Zemiathrips; a new genus of fungus-feeding phlaeothripine Thysanoptera in Australian leaf-litter. Australian Journal of Entomology, 41, 209-215. https://doi.org/10.1046/j.1440-6055.2002.00296.x

Mound, L.A. (2004) Australian long-tailed gall thrips (Thysanoptera, Phlaeothripinae, Leeuweniini), with comments on related Old World taxa. Australian Journal of Entomology, 43, 36-45. https://doi.org/10.1111/j.1440-6055.2004.00406.x

Mound, L.A. (2007) New Australian spore-feeding Thysanoptera (Phlaeothripidae - Idolothripinae). Zootaxa, 1604, 53-68. https://doi.org/10.11646/zootaxa.1604.1.5

Mound, L.A. (2009a) Anew genus and species of Scirtothrips genusgroup (Thysanoptera: Thripidae) from Kenya, intercepted by Australian quarantine. Zootaxa, 2210, 65-68. https://doi.org/10.11646/zootaxa.2210.1.4

Mound, L.A. (2009b) New taxa and new records of Australian Panchaetothripinae (Thysanoptera, Thripidae). Zootaxa, 2292, 25-33. https://doi.org/10.11646/zootaxa.2292.1.3

Mound, L.A. (2011) Grass-dependent Thysanoptera of the family Thripidae from Australia. Zootaxa, 3064, 1-40. https://doi.org/10.11646/zootaxa.3064.1.1

Mound, L.A. (2015) Problems in Costa Rican thrips taxonomy and systematics (Insecta, Thysanoptera). Florida Entomologist, 98, 27-31. https://doi.org/10.1653/024.098.0105

Mound, L.A. (2020) Covidthrips novendecim: an isolated new Phlaeothripinae taxon (Thysanoptera) from Queensland. Australian Entomologist, 47 (4), 248-252.

Mound, L.A., Cavalleri, A., O’Donnell, C., Infante, F., Ortix, J. \& Goldarazena, A. (2016) Ambaeolothrips: a new genus of Neotropical Aeolothripidae (Thysanoptera), with observations on the type-species from mango trees in Mexico. Zootaxa, 4132 (3), 413-421.

https://doi.org/10.11646/zootaxa.4132.3.9

Mound, L.A. \& Crespi, B.J. (1992) The complex of phlaeothripine thrips (Insecta, Thysanoptera) in woody stem galls of Casuarina in Australia. Journal of Natural History, 26, 395406.

https://doi.org/10.1080/00222939200770221

Mound, L.A., Heming, B.S. \& Palmer, J.M. (1980) Phylogenetic relationships between the families of recent Thysanoptera. Zoological Journal of the Linnean Society of London, 69, 111-141.

https://doi.org/10.1111/j.1096-3642.1980.tb01934.x
Mound, L.A. \& Houston, K.J. (1987) An annotated check-list of Thysanoptera from Australia. Occasional Papers on Systematic Entomology, 4, 1-28.

Mound, L.A. \& Marullo, R. (1993) The Erythrothrips complex of tropical Aeolothripidae (Thysanoptera) with a new Australian genus and a new South African species. Entomologica Scandinavica, 24, 285-291. https://doi.org/10.1163/187631293X00118

Mound, L.A. \& Marullo, R. (1994) New thrips on Mother-in-Law's Tongue. Entomologist's Monthly Magazine, 130, 95-98.

Mound, L.A. \& Marullo, R. (1996) The Thrips of Central and South America: An Introduction. Memoirs on Entomology, International, 6, 1-488.

Mound, L.A. \& Marullo, R. (1999) Two new basal-clade Thysanoptera from California with Old World affinities. Journal of the New York entomological Society, 106, 81-94. [https://www.biodiversitylibrary.org/page/50850636]

Mound, L.A. \& Masumoto, M. (2005) The genus Thrips (Thysanoptera, Thripidae) in Australia, New Caledonia and New Zealand. Zootaxa, 1020, 1-64.

https://doi.org/10.11646/zootaxa.1020.1

Mound, L.A. \& Masumoto, M. (2009) Australian Thripinae of the Anaphothrips genus-group (Thysanoptera), with three new genera and thirty-three new species. Zootaxa, 2042, 1-76. https://doi.org/10.11646/zootaxa.2042.1

Mound, L.A. \& Minaei, K. (2006) New fungus-feeding thrips (Thysanoptera-Phlaeothripinae) from tropical Australia. Zootaxa, 1150, 1-17. https://doi.org/10.11646/zootaxa.1150.1.1

Mound, L.A. \& Minaei, K. (2007) Australian insects of the Haplothrips lineage (Thysanoptera - Phlaeothripinae). Journal of Natural History, 41, 2919-2978. https://doi.org/10.1080/00222930701783219

Mound, L.A., Morison, G.D., Pitkin, B.R. \& Palmer, J.M. (1976) Thysanoptera. Handbooks for the Identification of British Insects, 1 (11), 1-79.

Mound, L.A. \& Moritz, G. (2000) Corroboreethrips; a new genus of minute apterous thrips (Insecta, Thysanoptera, Phlaeothripinae) from the bark of Australian Acacia trees. Invertebrate Taxonomy, 14, 709-716. https://doi.org/10.1071/IT00002

Mound, L.A. \& Morris, D.C. (2000) Inquilines or kleptoparasites? New phlaeothripine Thysanoptera (Insecta) associated with domicile-building thrips on Acacia trees. Australian Journal of Entomology, 39, 130-137. https://doi.org/10.1046/j.1440-6055.2000.00165.x

Mound, L.A. \& Morris, D.C. (2001) Domicile constructing phlaeothripine Thysanoptera from Acacia phyllodes in Australia: Dunatothrips Moulton and Sartrithrips gen.n., with a key to associated genera. Systematic Entomology, 26, 401-419. https://doi.org/10.1046/j.0307-6970.2001.00159.x

Mound, L.A. \& Morris, D.C. (2007) A new thrips pest of Myoporum cultivars in California, in a new genus of leaf-galling Australian Phlaeothripidae (Thysanoptera). Zootaxa, 1495, 35-45. https://doi.org/10.11646/zootaxa.1495.1.2

Mound, L.A. \& Ng, Y.-F. (2018) Autapomorphies in the generic classification of Plectrothripini (Thysanoptera, Phlaeothripinae), with a new genus and a new record from Malaysia. Zootaxa, 4402 (2), 390-394. 
https://doi.org/10.11646/zootaxa.4402.2.10

Mound, L.A. \& Nickle, D.A. (2009) The Old-World genus Ceratothripoides (Thysanoptera: Thripidae) with a new genus for related New-World species. Zootaxa, 2230, 57-63. https://doi.org/10.11646/zootaxa.2230.1.6

Mound, L.A. \& O’Neill, K. (1969) Physothrips Karny, 1912 (Insecta, Thysanoptera): Proposed designation of a typespecies under the plenary powers. N.Z. (S.) 1869. Bulletin of Zoological Nomenclature, 26, 51-53.

Mound, L.A. \& Palmer, J.M. (1974) Thrips rufa Gmelin, 1790 (Insecta, Thysanoptera, Thripidae): Proposed suppression under the Plenary Powers so as to validate T. rufa Haliday, 1836. Z.N. (S.) 2067 Bulletin of Zoological Nomenclature, 31, 228-229.

Mound, L.A. \& Palmer, J.M. (1981) Phylogenetic relationships between some genera of Thripidae (Thysanoptera). Entomologica Scandinavica, 15, 153-17.

Mound, L.A. \& Palmer, J.M. (1983a) The generic and tribal classification of spore-feeding Thysanoptera (Phlaeothripidae: Idolothripinae). Bulletin of the British Museum (Natural History). Entomology, 46, 1-174. [https://www. biodiversitylibrary.org/page/2355745]

Mound, L.A. \& Palmer, J.M. (1983b) An enigmatic fungus-feeding Thysanopteran from Singapore. Systematic Entomology, 8, 421-424.

https://doi.org/10.1111/j.1365-3113.1983.tb00492.x

Mound, L.A. \& Tree, D.J. (2009) Identification and host-plant associations of Australian Sericothripinae (Thysanoptera, Thripidae). Zootaxa, 1983, 1-22.

https://doi.org/10.11646/zootaxa.1983.1.1

Mound, L.A. \& Tree, D.J. (2011) Australian spore-feeding Thysanoptera of the genus Bactrothrips (Phlaeothripidae - Idolothripinae). Zootaxa, 3087, 56-65. https://doi.org/10.11646/zootaxa.3087.1.2

Mound, L.A. \& Tree, D.J. (2014a) Fungus-feeding phlaeothripine Thysanoptera in the genus Holothrips from Australia and New Caledonia, with a structurally similar new genus, Holoengythrips. Zootaxa, 3860 (2), 125-148. https://doi.org/10.11646/zootaxa.3860.2.2

Mound, L.A. \& Tree, D.J. (2014b) Generic relationships of two obscure Australian Thysanoptera species described by A.A.Girault. Australian Entomologist, 41(4), 205-210.

Mound, L.A. \& Tree, D.J. (2017) Two new Australian fungusfeeding thrips in two new Plectrothripini genera (Thysanoptera, Phlaeothripinae). Zootaxa, 4273 (3), 443-446. https://doi.org/10.11646/zootaxa.4273.3.10

Mound, L.A. \& Walker, A.K. (1982) Terebrantia (Insecta: Thysanoptera). Fauna of New Zealand, 1, 1-113.

Mound, L.A. \& Walker, A.K. (1986) Tubulifera (Insecta: Thysanoptera). Fauna of New Zealand, 10, 1-140.

Mound, L.A. \& Wells, A. (2007) A new genus for an Australian thrips (Thysanoptera, Phlaeothripinae) associated with a waxy eriococcid (Hemiptera, Coccoidea). Zootaxa, 1645, 57-61. https://doi.org/10.11646/zootaxa.1645.1.5

Mound, L.A. \& Wells, A. (2015) Endemics and adventives: Thysanoptera (Insecta) Biodiversity of Norfolk, a tiny Pacific Island. Zootaxa, 3964 (2), 183-210. https://doi.org/10.11646/zootaxa.3964.2.2

Mound, L.A. \& Wells, A. (2020) Two new monobasic thrips genera for a gall-inducing species and its kleptoparasite
(Thysanoptera, Phlaeothripinae). Zootaxa, 4759 (3), 421426.

https://doi.org/10.11646/zootaxa.4759.3.8

Müller, O.F. (1776) Zoologice Danica prodromus, seu animalium Danice et Norvegice indigenarum characteres, nomina, et synonyma imprimis popularium. Havniae, typis Hallageriis, $274 \mathrm{pp}$

https://doi.org/10.5962/bhl.title.13268

Muraleedharan, N. \& Sen, S. (1978) Two new species of Tubulifera (Thysanoptera: Phlaeothripidae) from N.E. India with the description of a new subgenus. Bulletin of the Zoological Survey of India, 1 (3), 257-261.

Nakahara, S. (1996a) Ewartithrips new genus (Thysanoptera: Thripidae) and four new species from California. Journal of the New York Entomological Society, 103, 229-250.

Nakahara, S. (1996b) Xerothrips dissimilis new genus and species (Thysanoptera: Thripidae) from California and Nevada. Proceedings of the entomological Society of Washington, 98, 208-214.

Nakahara, S. \& Foottit, R.G. (2012) Review of Chirothrips and related genera (Thysanoptera: Thripidae) of the Americas, with descriptions of one new genus and four new species. Zootaxa, 3251 (1), 1-29. https://doi.org/10.11646/zootaxa.3251.1.1

Nakahara, S., O’Donnell, C.A. \& Mound, L.A. (2015) Heliothrips haemorrhoidalis and its relatives, with one new species and one new genus (Thysanoptera). Zootaxa, 4021 (4), 578-584. https://doi.org/10.11646/zootaxa.4021.4.7

Ng, Y.-F. \& Mound, L.A. (2015) Genera of the Scirtothrips genusgroup (Thysanoptera, Thripidae) with a new species of Siamothrips from Malaysia. Zootaxa, 4021 (2), 387-394. https://doi.org/10.11646/zootaxa.4021.2.9

Nonaka, T. \& Okajima, S. (1992) Two new genera and species of the subfamily Panchaetothripinae (Thysanoptera, Thripidae) from Southeast Asia. Bulletin of the Biogeographical Society of Japan, 47, 103-108.

Nonaka, T. \& Jangvitaya, P. (1993) Bamboo-inhabiting thrips of the family Thripidae (Thysanoptera) from Southeast Asia I. Japanese Journal of Entomology, 61, 737-747.

Nonaka, T. \& Yangvitaya, P. (1994) Bamboo-inhabiting thrips of the family Thripidae (Thysanoptera) from Southeast Asia, II. Japanese Journal of Entomology, 62, 41-53.

Okajima, S. (1975) Notes on the Thysanoptera from the Ryukyu Islands. I. Descriptions of two new species. Kontyu, 43, 1319.

Okajima, S. (1976) Notes on the Thysanoptera from the Ryukyu Islands II. On the genus Stigmothrips Ananthakrishnan. Kontyu, 44, 119-129.

Okajima, S. (1978) Notes on the Thysanoptera from Southeast Asia IV. A new genus and two new species of the tribe Hyidiothripini (Phlaeothripidae). Kontyu, 46, 539-548.

Okajima, S. (1981) A revision of the tribe Plectrothripini of fungusfeeding Thysanoptera (Phlaeothripidae, Phlaeothripinae). Systematic Entomology, 6, 291-336. https://doi.org/10.1111/j.1365-3113.1981.tb00441.x

Okajima, S. (1983) Studies on some Psalidothrips species with key to the world species (Thysanoptera, Phlaeothripidae). Journal of Natural History, 17, 1-13.

https://doi.org/10.1080/00222938300770011

Okajima, S. (1984) Apelaunothripini from the Philippines 
(Thysanoptera, Phlaeothripidae). Journal of Natural History, 18, 717-738.

https://doi.org/10.1080/00222938400770611

Okajima, S. (1986) Studies on the genus Podothrips Hood from Taiwan, with description of a new species (Thysanoptera, Phlaeothripidae). Kontyu, 54, 713-718.

Okajima, S. (1987a) Studies on the Old World species of Holothrips (Thysanoptera, Phlaeothripidae). Bulletin of the British Museum (Natural History), Entomology, 54, 1-74. [https:// www.biodiversitylibrary.org/page/41033577]

Okajima, S. (1987b) Some Thysanoptera from the East Kalimantan, Borneo, with descriptions of a new genus and five new species. Transactions of the Shikoku Entomological Society, 18, 289-299.

Okajima, S. (1988) The genus Phylladothrips (Thysanoptera, Phlaeothripinae) from east Asia. Kontyu, 54, 706-722.

Okajima, S. (1989) Two thripine species collected on egg-plant in Thailand (Thysanoptera, Thripidae). Bulletin of the Biogeographical Society of Japan, 45, 71-75.

Okajima, S. (1995) A revision of the bamboo- and grass-inhabiting genus Bamboosiella Ananthakrishnan (Thysanoptera, Phlaeothripidae) II. Japanese Journal of Entomology, 63, 469-484.

Okajima, S. (1998) Minute leaf-litter thrips of the genus Preeriella (Thysanoptera, Phlaeothripidae) from Asia. Species Diversity, 3, 301-316. https://doi.org/10.12782/specdiv.3.301

Okajima, S. (2000) The first cycad-associated thrips in the family Phlaeothripidae, Stomothrips cycasi, gen. et sp. nov. (Thysanoptera). Invertebrate Taxonomy, 14, 705-708. https://doi.org/10.1071/IT00001

Okajima, S. (2002) Watanabeothrips yasuakii (Thysanoptera, Thripidae), a new genus and species from Thailand with remarkable sexual dimorphism in setal shape. Special Bulletin of the Japanese Society of Coleopterology, 5, 111-118.

Okajima, S. (2006) The Suborder Tubulifera (Thysanoptera). The Insects of Japan, 2, 1-720. The Entomological Society of Japan, Touka Shobo Co. Ltd., Fukuoka.

Okajima, S. \& Urushihara, H. (1992) Leaf-litter thrips found in Jinmuji Forest, the Miura Peninsula, Kanagawa Prefecture, Japan (Thysanoptera). Japanese Journal of Entomology, 60, $157-173$.

Okajima, S. \& Urushihara, H. (1997) Studies on some Allothripine thrips from tropical East Asia (Thysanoptera, Phlaeothripidae). Japanese Journal of Systematic Entomology, 3, 259-268.

O'Neill, K. (1960) Amynothrips andersoni, a new genus and species injurious to Alligatorweed (Thysanoptera: Phlaeothripidae). Proceedings of the Entomological Society of Washington, 70, 175-183.

O’Neill, K. (1967) Catinathrips, new genus, a new species, and a reassignment (Thysanoptera: Thripidae). Annals of the Entomological Society of America, 60, 854-856. https://doi.org/10.1093/aesa/60.4.854

Oustalet, M. (1873) Sur quelques espèces fossils de l'ordre des Thysanoptères. Bulletin de la Société Philomathique de Paris, 6 (10), 20-27.

Palmer, J.M. \& Mound, L.A. (1978) Nine genera of fungusfeeding Phlaeothripidae (Thysanoptera) from the Oriental Region. Bulletin of the British Museum (Natural History). Entomology, 37, 153-215. [https://www.biodiversitylibrary. org/page/2242974]

Palmer, J.M. \& Mound, L.A. (1985) New World Thripidae (Thysanoptera) with nine-segmented antennae. Zoological Journal of the Linnean Society, 84, 181-194.

https://doi.org/10.1111/j.1096-3642.1985.tb01798.x

Pelikán, J. (1947) Notice a la connaissance des Thysanoptères de la tourbière de Rejvíz. Entomologické Listy (Folia Entomologica) 10, 2-16. [in Czech].

Pelikán, J. (1949) A new subgenus and species of Thysanoptera from Czechoslovakia. Entomologické Listy (Folia Entomologica) $12,37-41$.

Pelikán, J. (1958) Beiträge zur Kenntnis der Thysanopteren Bulgariens - I. Práce Brněnské základny Československé akademie věd, 30, 423-433.

Pelikán, J. (1963a) New Thysanoptera from Central Asia (U.S.S.R.). Časopis Československé Společnosti Entomologické (Acta Societatis Entomologicae Cechosloveniae), 60, 99-113.

Pelikán, J. (1963b) A new Haplothrips from China. Časopis Československé Společnosti Entomologické (Acta Societatis Entomologicae Cechosloveniae), 60, 273-276.

Pelikán, J. (1968) Two new Thysanoptera from Asia with notes on synonymy. Acta Entomologica Bohemoslovaca, 65, 216 221.

Pelikán, J. (1970) Thysanopteren aus Nepal (Ins., Thysanoptera). Khumbu - Himal (Universitäts Verlag Innsbruck - München), 3, 361-370.

Pelikán, J. (1984) Thysanopteren aus der Mongolei - III. Annales Historico-Naturales Musei Nationalis Hungarici, 76, 109128.

Pelikán, J. (2001) Another pugnacious thrips, Tragothrips kubani gen. nov. and sp. nov., from Laos (Thysanoptera, Phlaeothripidae). Acta Musei Moraviae, Scientiae biologicae (Brno), 86, 171-175.

Pelikán, J. (2004) Tumidothrips spiniceps gen. et sp. nov., a new thrips from Laos (Thysanoptera, Phlaeothripidae, Idolothripinae). Acta Musei Moraviae, Scientiae biologicae (Brno), 89, 19-23.

Peñalver, E., Conrad, C., Labandeira, C.C., Barrón, E., Delclòs, X., Nel, P., Nel, A., Tafforeau, P., \& Soriano, C. (2012) Thrips pollination of Mesozoic gymnosperms. Proceedings of the National Academy of Sciences, 109, 8623-8628. https://doi.org/10.1073/pnas.1120499109

Peñalver, E. \& Nel, P. (2010) Hispanothrips from Early Cretaceous Spanish amber, a new genus of the resurrected family Stenurothripidae (Insecta: Thysanoptera). Annales de la Société entomologique de France (N.S.), 46 (1-2), 138-147. https://doi.org/10.1080/00379271.2010.10697649

Pergande, T. (1891) Footnote in Duffey, J.C. (1891) "Transformation of a carabid (Plochionus timidus), and observations on a coccinellid enemy of the red spider". Transactions of the Academy of Sciences of St Louis, 5 (3-4), 533-542.

Pergande, T. (1896) Description of a new species of Idolothrips. Entomological News, 7, 63-64.

Pitkin, B.R. (1972) A revision of the Australian genus Odontothripiella Bagnall, with descriptions of fourteen new species (Thysanoptera: Thripidae). Journal of the Australian Entomological Society, 11, 265-289. https://doi.org/10.1111/j.1440-6055.1972.tb01631.x

Pitkin, B.R. (1973) Larothrips dentipes (Thysanoptera, Thripidae), a new genus and species of thrips from yam flowers in Nigeria. 
Bulletin of entomological Research, 62, 415-418. https://doi.org/10.1017/S000748530000393X

Pitkin, B.R. (1976) A revision of the Indian species of Haplothrips and related genera (Thysanoptera: Phlaeothripidae). Bulletin of the British Museum (Natural History) (Entomology), 34, 221280. [https://www.biodiversitylibrary.org/page/41005098]

Pitkin, B.R. (1977) The genus Antillothrips Stannard, with descriptions of two new species (Thysanoptera: Phlaeothripidae). Systematic Entomology, 2, 53-58. https://doi.org/10.1111/j.1365-3113.1977.tb00360.x

Pitkin, B.R. (1978) A revision of the Australian species of Anaphothrips Uzel (Thysanoptera: Thripidae). Australian Journal of Zoology, 26, 349-371. https://doi.org/10.1071/ZO9780349

Pitkin, B.R. \& Mound, L.A. (1973) A catalogue of West African Thysanoptera. Bulletin de l'Institut Fondamental de l'Afrique Noire, 35, 407-449.

Priesner, H. (1914) Neue Thysanopteren (Blasenfüße) aus Österreich. Entomologische Zeitung, Frankfurt, 27, 259-261; 265-266.

Priesner, H. (1919a) Zur Thysanopteren-Fauna Albaniens. Sitzungsberichte der Kaiserlichen Akademie der Wissenschaften, Wien, 128, 115-144.

Priesner, H. (1919b) Zur Thysanopteren-Fauna der ostadriatischen Küstenländer. Zeitschrift des Österreichischen Entomologischen Vereins, 4, 79-80; 89-90; 96-97; 104-106; 113-114.

Priesner, H. (1920a) Kurze Beschreibung neuer Thysanopteren aus Österreich. Sitzungsberichte der Kaiserlichen Akademie der Wissenschaften, Wien, 129, 71-88.

Priesner, H. (1920b) Beitrag zur Kenntnis der Thysanopteren Oberösterreichs. Jahresberichte Museum Francisco Carolinus, 78, 50-63.

Priesner, H. (1921a) Neue Europäische Thysanopteren. Wiener Entomologische Zeitung, 38, 115-122.

Priesner, H. (1921b) Haplothrips-Studien. Treubia, 2, 1-20.

Priesner, H. (1921c) Neue und wenig bekannte Thysanopteren der neotropischen Fauna aus der Sammlung des Berliner Zoologischen Museums. Deutsche entomologische Zeitung, 1921, 187-223.

https://oi.org/10.1002/mmnd.48019210309

Priesner, H. (1922a) Neue Rasenthripse aus Österreich. Konowia, 1, 87-96.

Priesner, H. (1922b) Beiträge zur Lebensgeschichte der Thysanopteren. II. Rhopalandrothrips obscurus (Uz.), Taeniothrips salicis (Rt.) und Taeniothrips dianthi Pr. Sitzungsberichte der Kaiserlichen Akademie der Wissenschaften, Wien, 131, 67-75.

Priesner, H. (1923) Ein Beitrag zur Kenntnis der Thysanopteren Surinams. Tijdschrifte voor Entomologie, 66, 88-111.

Priesner, H. (1924a) Neue Phloeothripiden (Thys.) aus Ungarn. Entomologische Zeitung, Frankfurt, 37, 49; 52-53; 38, 2-3.

Priesner, H. (1924b) Bernstein-Thysanopteren. Entomologische Mitteilungen, 13, 130-151. https://doi.org/10.1002/mmnd.192519250104

Priesner, H. (1924c) Neue Thysanopteren. Sitzungsberichte der Kaiserlichen Akademie der Wissenschaften, Wien, 133, 527542.

Priesner,H.(1925a)Zweineue, beachtenswerte Thysanopterentypen aus Ungarn. Zeitschrift des Österreichischen Entomologischen
Vereins, 10, 5-7.

Priesner, H. (1925b) Neue Thysanopteren. Deutsche entomologische Zeitschrift, 1925, 13-28.

https://doi.org/10.1002/mmnd.48019250104

Priesner, H. (1925c) Thysanopterologica I. Zoologische Jahrbücher, $50,305-319$.

Priesner, H. (1925d) Katalog der europäischen Thysanoptera. Konowia, 4, 141-159.

Priesner, H. (1926a) Die Thysanopteren Europas . Abteilung I-II: 1-342. F. Wagner Verlag, Wien.

Priesner, H. (1926b) Thysanopterologica II. Zoologische Jahrbücher, 52, 267-278.

Priesner, H. (1926c) Die Jugendstadien der malayischen Thysanopteren. Treubia 8 (suppl.), 1-264.

Priesner, H. (1926d) Un género nuevo y curioso del Orden Physopodos o Thysanópteros (insectos) de México. Memorias y Revista de la Sociedad Cientifica ,Antonia Alzate ', 44, 485489.

Priesner, H. (1927) Die Thysanopteren Europas. Abteilung III, 343-568. F. Wagner Verlag, Wien.

Priesner, H. (1928a) Die Thysanopteren Europas. Abteilung IV, 569-755. F. Wagner Verlag, Wien.

Priesner, H. (1928b) Über australische Thysanopteren. Sitzungsberichte der Kaiserlichen Akademie der Wissenschaften, Wien, 137, 643-659.

Priesner, H. (1928c) Beiträge zur Kenntnis der europäischen Thysanopteren. Konowia, 7, 322-325.

Priesner, H. (1928d) Verzeichnis der Thysanopteren Ungarns. Annales Museum nationalis Hungarici, 25, 60-68.

Priesner, H. (1929a) Bernstein-Thysanopteren II. BernsteinForschungen, 1, 111-138.

Priesner, H. (1929b) Indomalayische Thysanopteren I. Treubia, 10, 447-462.

Priesner, H. (1929c) Spolia Metawiensia: Thysanoptera. Treubia, $11,187-210$.

Priesner, H. (1930a) Contribution towards a knowledge of the Thysanoptera of Egypt, II. Bulletin de la Société Royal Entomologique d'Égypte, 13, 211-219.

Priesner, H. (1930b) Contribution towards a knowledge of the Thysanoptera of Egypt, III. Bulletin de la Société Royal Entomologique d'Égypte, 14, 6-15.

Priesner, H. (1931a) Review of the African Haplothrips-species (Thysanoptera). Bulletin de la Société Royal Entomologique d'Egypte, 14, 230-277.

Priesner, H. (1931b) Contribution towards a knowledge of the Thysanoptera of Egypt, IV. Bulletin de la Société Royal Entomologique d'Égypte, 15, 127-131.

Priesner, H. (1931c) Ein neues Genus aus der Familie Urothripidae. Konowia, 10, 93-95.

Priesner, H. (1931d) Ein neuer Blasenfuss, der Gallen an Euphorbia hirta verursacht. Miscellania Zoologica Sumatrana, 58, 1-4.

Priesner, H. (1932a) Contribution towards a knowledge of the Thysanoptera of Egypt, V. Bulletin de la Société Royal Entomologique d'Égypte, 16, 2-12.

Priesner, H. (1932b) Contribution towards a knowledge of the Thysanoptera of Egypt, VII. Bulletin de la Société Royal Entomologique d'Égypte, 16, 45-51.

Priesner, H. (1932c) Indomalayische Thysanopteren IV [Teil 1]. Konowia, 11, 49-64.

Priesner, H. (1932d) Drei neue Thripiden. Stylops, 1, 108-111. 
https://doi.org/10.1111/j.1365-3113.1932.tb01365.x

Priesner, H. (1932e) Thysanopteren aus dem Belgischen Congo. Revue zoologie et botanique africaines, 22, 192-221.

Priesner, H. (1933a) Neue Thysanopteren aus Mexico, gesammelt von Prof. Dr. A. Dampf. Teil II. Wiener Entomologische Zeitung, 50, 49-83.

Priesner, H. (1933b) Neue exotische Thysanopteren. Stylops, 2, $145-156$. https://doi.org/10.1111/j.1365-3113.1993.tb00991.x

Priesner, H. (1933c) Indomalayische Thysanopteren IV. [Teil 2]. Konowia, 12, 69-85; 307-318.

Priesner, H. (1933d) Contributions towards a knowledge of the Thysanoptera of Egypt, VIII. Bulletin de la Société Royal Entomologique d'Égypte, 17, 1-7.

Priesner, H. (1934a) Contributions towards a knowledge of the Thysanoptera of Egypt, IX. Bulletin de la Société Royal Entomologique d'Égypte, 18, 275-285.

Priesner, H. (1934b) Indomalayische Thysanopteren VII. [as VI]. Natuurkundig Tijdschrift voor Nederlandsch Indie, 94, 254290.

Priesner, H. (1935a) Indomalayische Thysanopteren VI. [as VII]. Konowia, 14, 58-339.

Priesner, H. (1935b) Neue exotische Thysanopteren. Stylops, 4, $125-131$. https://doi.org/10.1111/j.1365-3113.1935.tb00574.x

Priesner, H. (1935c) New or little-known oriental Thysanoptera. Philippine Journal of Science, 57, 351-375.

Priesner, H. (1935d) On some Thysanoptera from Cyprus. Bulletin de la Société Royal Entomologique d'Ègypte, 19, 308-314.

Priesner, H. (1936a) Über einige neue wenig bekannte Thysanopteren. Proceedings of the Royal entomological Society of London (B), 5, 208-214. https://doi.org/10.1111/j.1365-3113.1936.tb00587.x

Priesner, H. (1936b) A preliminary review of the non-fossil species of the genus Melanthrips Hal. (Thysanoptera). Bulletin de la Société Royal Entomologique d'Égypte, 20, 29-52.

Priesner, H. (1936c) On some further new Thysanoptera from the Sudan. Bulletin de la Société Royal Entomologique d'Égypte, 20, 83-104.

Priesner, H. (1938a) On the "Sugarcane Thrips" Podothrips lucasseni (Krüg.), and some allied species (Thysanoptera). Bulletin de la Société Royal Entomologique d'Égypte, 21, 68-81.

Priesner, H. (1938b) Thysanopteren aus dem Belgischen Congo (4. Beitrag). Revue de zoologie et botanique africaine, 30, 343-355.

Priesner, H. (1938c) Thysanopterologica VI. Konowia, 17, $29-35$. Priesner, H. (1938d) Materialen zu einer Revision der TaeniothripsArten (Thysanoptera) des indomalayischen Faunengebietes. Treubia, 16, 469-526.

Priesner, H. (1939a) Contributions towards a knowledge of the Thysanoptera of Egypt, XII. Bulletin de la Société Royal Entomologique d'Égypte, 22, 123-132.

Priesner, H. (1939b) Thysanopterologica VIII. Proceedings of the Royal entomological Society of London, (B), 8, 73-78. https://doi.org/10.1111/j.1365-3113.1939.tb00495.x

Priesner, H. (1939c) Thysanopteren aus dem Belgischen Congo (6. Beitrag). Revue de zoologie et botanique africaine, 32, $154-175$

Priesner, H. (1940a) Thysanopterologica IX. Philippine Journal of
Science, 41, 403-409.

Priesner, H. (1940b) On some Thysanoptera (Thripidae) from Palestine and Cyprus. Bulletin de la Société Royal Entomologique d'Egypte, 24, 46-56.

Priesner, H. (1949a) Thysanopterologica, X. Revue française d'entomologie, 16, 93-96.

Priesner, H. (1949b) Genera Thysanopterorum. Keys for the identification of the genera of the order Thysanoptera. Bulletin de la Société Royal Entomologique d'Égypte, 33, 31-157.

Priesner, H. (1950a) Contributions towards a knowledge of the Thysanoptera of Egypt, XV. Bulletin de la Société Royal Entomologique d'Égypte, 34, 25-37.

Priesner, H. (1950b) Further studies in Haplothrips and allied genera (Thysanoptera). Bulletin de la Société Royal Entomologique d'Egypte, 34, 69-120.

Priesner, H. (1951) Thysanopterologica, XI. Annals and Magazine of Natural History, (12) 4, 355-371. https://doi.org/10.1080/00222935108654160

Priesner, H. (1952a) On some new genera and species of Thysanoptera from the Oriental region. Indian Journal of Entomology, 13, 183-200.

Priesner, H. (1952b) On some Central African Thysanoptera. Bulletin de l'Institut Fondamental de l'Afrique Noire, 14, 842-880.

Priesner, H. (1953) On the genera allied to Liothrips of the Oriental fauna, I. (Thysanoptera). Treubia, 22, 357-380. https://doi.org/10.14203/treubia.v27i2-3.1559

Priesner, H. (1957) Zurvergleichenden Morphologie des Endothorax der Thysanopteren. Zoologischer Anzeiger, 159, 159-167.

Priesner, H. (1961) Über einige Thysanopteren aus der Türkei. Verhandlungen des Vereins für naturwissenschaftliche Heimatforschung, 35, 16-24.

Priesner, H. (1964) Ordnung Thysanoptera (Fransenflügler, Thripse). In Franz H., Bestimmungsbücher zur Bodenfauna Europas, 2, 1-242. Akademie-Verlag.

Priesner, H. (1965) A monograph of the Thysanoptera of the Egyptian deserts. Publications de l'Institut Desert d'Égypte, $13,1-549$.

Priesner, H. (1968) On the genera allied to Liothrips of the Oriental fauna II (Insecta-Thysanoptera). Treubia, 27, 175-285.

Priesner, H. \& Seshadri, A.R. (1952) Some new Thysanoptera from South India. Indian Journal of agricultural Sciences, 22, 405-411.

Raizada, U. (1966) Studies on some Thysanoptera from Delhi. Zoologischer Anzeiger, 176, 277-290.

Ramakrishna, T.V. (1928) A contribution to our knowledge of the Thysanoptera of India. Memoirs of the Department of Agriculture of India (Entomology Series 10), 7, 217-316

Ramakrishna, T.V. (1934) Notes on Indian Thysanoptera with descriptions of new species. Records of the Indian Museum, 36, 491-498.

Ramakrishna, T.V. (1935) A new species of Thysanoptera from S. India (Taeniothrips cardamomi, sp. nov.). Memoirs of the Department of Agriculture in India. Entomology Series, 7, 258-265.

Ramakrishna, T.V. \& Margabandhu, V. (1931) Notes on Indian Thysanoptera with brief descriptions of new species. Journal of the Bombay Natural History Society, 34, 1025-1040.

Ramakrishna, T.V. \& Margabandhu, V. (1939) Notes on new and known Indian Thysanoptera. Records of the Indian Museum, 
$41,21-33$.

Retana-Salazar, A.P. (2000) Revisión y filogenia del grupo generico Pseudothrips (Thysanoptera: Thripidae). Brenesia, 54, 5162.

Retana-Salazar, A.P. (2007a) El grupo genérico Hoodothrips (Terebrantia: Heliothripidae). Acta Zoológica Lilloana, 51 (1), 15-38.

Retana-Salazar, A.P. (2007b) Los tisanopteros del grupo genérico Anaphothrips (Thysanoptera: Thripidae) con énfasis en America Central. Revista de Biología Tropical, 55 (1), 321333. https://doi.org/10.15517/rbt.v55i1.6084

Retana-Salazar, A.P. (2009) Monografía de lo Grupos Genéricos Anactinothrips-Zeugmatothrips (Tubulifera: Idolothripinae). Editorial Fundación Instituto Centroamericano para la Investigación en Biología y Conservación (ECIBRC). Costa Rica. 140pp.

Retana-Salazar,A. \& Mound, L.A. (2005) Character state variation in a new genus and species of Thripidae (Insecta: Thysanoptera) associated with Chamaedorea (Arecaceae) inflorescences in Central America. Brenesia, 63-64, 121-126.

Retana-Salazar, A.P. \& Nishida, K. (2007) First gall-inducing thrips on Elaphoglossum ferns: A new genus and species of thrips, Jersonithrips galligenus from Costa Rica (Insecta, Thysanoptera, Phlaeothripidae). Senckenbergiana biologica, 87 (2), 143-148.

Retana-Salazar, A.P. \& Soto-Rodríguez, G.A. (2007) Revisión Taxonómica del grupo Haplothrips-Karnyothrips (Thysanoptera: Phlaeothripidae). Revista de Biología Tropical, 55, 627-635. https://doi.org/10.15517/rbt.v55i2.6037

Retana-Salazar, A.P. \& Soto-Rodríguez, G.A. (2008) A new genus and species of gall-inducing thrips (Thysanoptera: Tubulifera) from the neotropical region. Brenesia, 69, 59-64.

Retana-Salazar, A.P. \& Soto-Rodríguez, G.A. (2013a) Un nuevo género de thrips con estiletes maxilares anchos (Phlaeothripidae: Tubulifera). Métodos en Ecología y Sistemática, 8 (1), 64-69.

Retana-Salazar, A.P. \& Soto-Rodríguez, G.A. (2013b) Un nuevo género áptero de thrips de Costa Rica (Tubulifera: Phlaeothripidae: Phlaeothripinae). Métodos en Ecología y Sistemática, 8 (1), 70-80.

Reuter, O.M. (1879) Diagnoser ofver nya Thysanoptera fran Finland. Ofversigt of Finska Vetenskaps-Societetens Forhaandlingar, 21, 207-223.

Reuter, O.M. (1880a) Thysanoptera Fennica. I. Tubulifera. Bidrag till Kannedom af Finlands Natur och Folk, 40, 1-26.

Reuter, O.M. (1880b) A New Thysanopterous Insect of the Genus Phloeothrips Found in Scotland and Described. The Scottish Naturalist, 5, 310-311.

Reuter, O.M. (1884) Phloeothrips albosignata n. sp. ex Algeria. Revue d'Entomologie, Caen, 3 (10), 290-291.

Reuter, O.M. (1891) Thysanoptera, funna I finska orangerier. Meddelelser af Societas pro Fauna et Flora Fennica, 17, 161-167.

Reuter, O.M. (1899) Thysanoptera Fennica. Acta Societas pro Fauna et Flora Fennica, 17 (2), 1-67.

Reuter, O.M. (1901) Thysanoptera tria Mediterranea. Finska Vetenskaps-Societetens Förhandlingar, 43, 214-216.

Reuter, O.M. (1904) Eine neues Warmhaus-Thysanopteron.
Meddelelser af Societas pro Fauna et Flora Fennica, 30, 106-109.

https://doi.org/10.5962/bhl.part.17433

Reyes, C.P. (1994) Thysanoptera (Hexapoda) of the Philippine Islands. The Raffles Bulletin of Zoology, 42, 107-507.

Richter, W. (1928) Beitrag zur Kenntnis der Aeolothripiden (Thysanoptera). Deutsche Entomologische Zeitschrift, 1928, 29-37.

https://doi.org/10.1002/mmnd.192819280103

Ruiz-De la Cruz, J., Vasquez-Lopez, A., Retana-Salazar, A.P., Mora-Aguilera, J.A. \& Johansen-Naime, R. (2013) A new species of Aeolothrips (Thysanoptera: Aeolothripidae) from mango crops in Oaxaca, Mexico. Florida Entomologist, 96 (1), 29-35. https://doi.org/10.1653/024.096.0104

Sakimura, K. (1967) A preliminary note on a review of the genus Neurisothrips new genus. Proceedings of the Hawaiian entomological Society, 19, 419-423.

Sakimura, K. (1968) A preliminary review of the genus Homochaetothrips gen.nov., with brief description of a new species. Journal of the Australian entomological Society, 7, 59-62.

https://doi.org/10.1111/j.1440-6055.1968.tb00702.x

Sakimura, K. \& Bianchi, F.A. (1977) A review of the Hawaiian species of Idolothripinae (Phlaeothripidae: Thysanoptera). Proceedings of the entomological Society of Hawaii, 22, 495-523.

Savenko, R.F. (1941) Neue Thysanopteren aus Georgien. Mitteilungen der Akademie der Wissenschaften der Georgischen SSR, 2 (4), 369-371.

Schille, F. (1911) Nowe forme Przylzencow. (Thysanopterorum genera et species novae). Sprawozdanie Komisyi Fizyograficznej, Krakowie, 45, 3-10.

Schlechtendal, D. von (1887) Physopoden aus dem Braunkohlengebirge von Rott am Siebengebirge. Zeitschrift für Naturwissenschaften, 60, 551-592.

Schliephake, G. (1972) Systematische und morphologisch vergleichende Beziehungen der europäischen Genera der Subtribus Thripina (Thysanoptera). Folia Entomologica Hungarica, 25, 271-277.

Schliephake, G. (1975) Beitrag zur phylogenetischen Systematik bei Thysanoptera (Insecta). Beiträge zur Entomologie, 25, 5-13.

Schliephake, G. (1993) Beiträge zur Kenntnis fossiler Fransenflügler (Thysanoptera, Insecta) aus dem Bernstein des Tertiär. 2. Beitrag: Aeolothripidae (Melanthripinae) und Thripidae (Dendrothripinae und Thripinae). Zoologische Jahrbücher: Abteilung für Systematik, Ökologie und Geographie der Tiere, Jena, 120, 215-251.

Schliephake, G. (1999) Fossil Thrips (Thysanoptera, Insecta) of the Baltic (North- and Baltic Sea) and Saxonian (Bitterfeldian) Tertiary Amber from the Collections of Hoffeins. Mitteilungen des Museums für Naturkunde in Berlin. Deutsche entomologische Zeitschrift (Berlin), 46, 1, 83-100. https://doi.org/10.1002/mmnd.4800460105

Schliephake, G. (2000) Neue Fransenflügler aus dem Baltischen und Bitterfelder Bernstein (Insecta: Thysanoptera). Mitteilungen aus dem Geologisch-Paläontologischen Institut der Universität Hamburg, 84, 219-230.

Schliephake, G. (2001a) Weitere neue Funde fossiler Fransenflügler 
aus dem Baltischen Bernstein (Insecta, Thysanoptera). Mitteilungen aus dem Geologisch-Palaeontologischen Institut der Universität Hamburg, 85, 197-201.

Schliephake, G. (2001b) Thysanoptera (Insecta) of the Tertiary amber of the Museum of the Earth, Warsaw, with keys to the species of the Baltic and Bitterfeld amber. Prace Muzeum Ziemi, 46, 17-39.

Schliephake, G. (2003) Fossile Thysanoptera (Insecta) aus dem Baltischen Bernstein. Mitteilungen aus dem GeologischPaläontologischen Institut der Universität Hamburg, 87, 171-182.

Schmutz,K.(1909)ZurKenntnis einigerneuen Thysanopterengenera (Tubulifera). Annalen des Naturhistorischen Museums in Wien, 23, 273-281.

Schmutz, K. (1913) Zur Kenntnis der Thysanopterenfauna von Ceylon. Sitzungsberichte der Kaiserlichen Akademie der Wissenschaften, Wien, 122 (7), 991-1089.

Scudder, S.H. (1867) The results of an examination of a small collection of fossil insects obtained by Prof. William Denton in the Tertiary Beds of Green River, Colorado. Proceedings of the Boston Society of Natural History, 11, 117-118.

Scudder, S.H. (1875) The Tertiary Physopoda of Colorado. Bulletin of the United States Geological and Geographical Survey of the Territories, 1 (4), 221-223.

Sen, S. \& Muraleedharan, N. (1976) New genera and species of Tubulifera (Thysanoptera: Phlaeothripidae) from Assam and Meghalaya. Entomon, 1 (2), 175-183.

Seshadri, A. \& Ananathakrishnan, T.N. (1954) Some new Indian Thysanoptera - 1. Indian Journal of Entomology, 16, 210226.

Sharov, A.G. (1972) On the phylogenetic relations of the order Thripida (Thysanoptera). Entomological Review, 54, 854858. [in Russian]

Shmakov, A.S. (2009) The oldest members of the families Aeolothripidae and Thripidae (Insecta: Thysanoptera) from the Lower Cretaceous of Transbaikalia. Paleontological Journal, 43 (4), 428-432.

https://doi.org/10.1134/S003103010904011X

Shmakov, A.S. (2014) Thrips (Insecta: Thysanoptera) from the Insect Limestone (Bembridge Marls, Late Eocene) of the Isle of Wight, UK. Earth and Environmental Science Transactions of the Royal Society of Edinburgh, 104, 317-325. https://doi.org/10.1017/S1755691014000097

Shull, A.F. (1909) Some apparently new Thysanoptera from Michigan. Entomological News, 20, 220-228.

Shumsher, S. (1942) Contribution to our knowledge of Indian Thysanoptera. Indian Journal of Entomology, 4 (2), 111135.

Shumsher, S. (1944) Studies on some Indian Thysanoptera. Proceedings of the Royal Entomological Society of London B, 13 (11-12), 139-144. https://doi.org/10.1111/j.1365-3113.1944.tb00774.x

Shumsher, S. (1946) Studies on the systematics of Indian Thysanoptera-Terebrantia. Indian Journal of Entomology, 7, 147-188.

Solowiow, P. (1924) Neue Arten Blasenfüße. Entomologische Zeitschrift, 38 (12/13), 25.

Soto-Rodríguez, G.A., Nishida, K. \& Retana-Salazar, A.P. (2012) A new genus of gall-dwelling thrips from Costa Rica (Thysanoptera: Phlaeothripidae: Phlaeothripinae). Revista
Mexicana de Biodiversidad, 83 (3), 605-610.

https://doi.org/10.22201/ib.20078706e.2012.3.1254

Soto-Rodríguez, G.A., Retana-Salazar, A.P. \& Rodríguez Arrieta, J.A. (2013) Dos géneros nuevos de thrips (Thysanoptera: Tubulifera) para Costa Rica. Acta zoológica lilloana, 57 (1), 31-41.

Stannard, L.J. (1950) A new genus of Phlaeothripidae from Mexico (Thysanoptera). Proceedings of the Entomological Society of Washington, 52, 143-146.

Stannard, L.J. (1952a) A new Australian Phlaeothripidae (Thysanoptera: Tubulifera). Proceedings of the Biological Society of Washington, 66, 127-130.

Stannard, L.J. (1952b) Peanut-winged thrips (Thysanoptera: Thripidae). Annals of the Entomological Society of America, 45, 327-330.

https://doi.org/10.1093/aesa/45.2.327

Stannard, L.J. (1953a) Illinothrips rossi, new genus and species (Thysanoptera: Phlaeothripidae). Illinois Academy of Science Transactions, 46, 193-196.

Stannard, L.J. (1953b) The genus Halmathrips Hood (Thysanoptera, Thripidae). Proceedings of the Entomological Society of Washington, 55, 1-6.

Stannard, L.J. (1953c) New Jamaican tubuliferan thrips (Thysanoptera: Phlaeothripidae). Occasional Papers of the Museum of the Institute of Jamaica, 6, 1-4.

Stannard, L.J. (1954) Actinothrips (Hybridothrips) oneillae, new subgenus and species (Thysanoptera: Phlaeothripidae). Proceedings of the Entomological Society of Washington, 56, 71-74.

Stannard, L.J. (1955) On some reticulate-headed genera of the tribe Glyptothripini Priesner (Thysanoptera: Phlaeothripidae). Transactions of the American Entomological Society, 81, 77-101.

Stannard, L.J. (1956) Five new thrips from the Southwest (Thysanoptera: Tubulifera). Proceedings of the Biological Society of Washington, 69, 21-28.

Stannard, L.J. (1957) The phylogeny and classification of the North American genera of the sub-order Tubulifera (Thysanoptera). Illinois Biological Monographs, 25, 1-200.

https://doi.org/10.5962/bhl.title.50287

Stannard, L.J. (1958) Two new and two rare Tubuliferous thrips, recorded principally from Illinois (Thysanoptera, Phlaeothripidae). Proceedings of the Entomological Society of Washington, 60, 271-275.

Stannard, L.J. (1960) A new genus and species of thrips from Equatorial Africa (Thysanoptera: Phlaeothripidae). Bulletin of Entomology, Madras, 1, 11-13.

Stannard, L.J. (1961) A new species of Allelothrips from South India, with notes on the synonymy and characteristics of the genus (Thysanoptera: Aeolothripidae). Bulletin of Entomology, Madras, 2, 9-11.

Stannard, L.J. (1962) Cartomothrips, a new genus based on two species from the Australian region (Thysanoptera: Phlaeothripidae). Proceedings of the Royal entomological Society of London. (B), 31, 38-40.

https://doi.org/10.1111/j.1365-3113.1962.tb01177.x

Stannard, L.J. (1963a) Insects of Macquarie Island. Thysanoptera: Thripidae. Pacific Insects, 4, 933-936.

Stannard, L.J. (1963b) New genera and species of Thysanoptera (Tubulifera: Phlaeothripidae) from Illinois and Alabama. 
Transactions of the Illinois State Academy of Science, 56, 134-138.

Stannard, L.J. (1968a) The thrips, or Thysanoptera, of Illinois Bulletin of the Illinois Natural History Survey, 29, 213-552. https://doi.org/10.21900/j.inhs.v29.166

Stannard, L.J. (1968b) Mixothrips, a new genus of gall making thrips (Thysanoptera: Phlaeothripidae). The Florida Entomologist, $51,135-141$ https://doi.org/10.2307/3493546

Stannard, L.J. (1970) New genera and species of Urothripini (Thysanoptera: Phlaeothripidae). Proceedings of the Royal entomological Society of London (B), 39, 114-124. https://doi.org/10.1111/j.1365-3113.1970.tb00264.x

Stannard, L.J. (1972) Three new species and one new genus of Heterothripidae (Thysanoptera) from South America. Proceedings of the Biological Society of Washington, 84, 335-344.

Stannard, L.J. \& Mitri, T.J. (1962) Preliminary studies on the Tryphactothrips complex in which Anisopilothrips, Mesostenothrips and Elixothrips are erected as new genera (Thripidae: Heliothripinae). Transactions of the American entomological Society, 88, 183-224.

Sulzer, J.H. (1776) Abgekürzte Geschichte der Insecten nach dem Linnaeischen System. Erster Theil. pp. 1-28, 1-274. Winterthur (H. Steiner \& Co.).

Targioni-Tozzetti, G. (1887) Notizie sommarie di due specie di Cecidomidei, una consociata ad un Phytoptus, ad altri acari e ad una Thrips in alcune galle del Nocciolo (Corylus avellana L.), una gregaria sotto la scorza dei rami di Olivo, nello stato larvale. Bollettino della Società Entomologica Italiana, 18 (4), 419-431. [https://www.biodiversitylibrary.org/item/ 87992\#page/524/mode/1up]

ThripsWiki (2021) ThripsWiki-providing information on the World's thrips. Available from: http://thrips.info/wiki/Main Page (accessed 7.i.2021)

Titschack, E. (1955) Thysanoptera XI. Watsoniella bonessi n.sp., ein neue Blasenfuß aus der Umgebung Kiels. Bombus, 1 (8687), 361.

Titschack, E. (1957) Thysanoptera XVI. Neufunde aus unserem faunistischen Arbeitsgebiet. Bombus, 1 (97-98), 407-410.

Titschack, E. (1958) Zwei neue Thysanopteren aus Südeuropa. Verhandlungen des Vereins für naturwissenschaftliche Heimatforschung, 33, 4-15.

Tong, T.T., Shih, C.K. \& Ren, D. (2019) A new genus and species of Stenurothripidae (Insecta: Thysanoptera: Terebrantia) from mid-Cretaceous Myanmar amber. Cretaceous Research, 100, 184-191. https://doi.org/10.1016/j.cretres.2019.03.005

Tong, X.-L. \& Liu, L. (2019) Yaothrips as a new synonym of Pezothrips, with description of the male of Pezothrips pediculae comb.n. (Thysanoptera: Thripidae). Zootaxa, 4646 (3), 598-600.

https://doi.org/10.11646/zootaxa.4646.3.12

Tree, D. \& Mound, L.A. (2009) Gall-induction by an Australian insect of the family Thripidae (Thysanoptera: Terebrantia). Journal of Natural History, 43, 1147-1158. https://doi.org/10.1080/00222930902807767

Tree, D. \& Mound, L.A. (2013) Leichhardt's vanishing thrips: a remarkable new genus and species of Insecta, Thysanoptera, Phlaeothripinae. Memoirs of the Queensland Museum, 58,
$375-379$.

Trybom, F. (1894) Iakttagelser om Blåsfotingar (Physapoder) från Sommaren 1893. Entomologisk Tidskrift, 15, 41-58.

Trybom, F. (1895) Iakttagelser om vissa blåsfotingars (physapoders) uppträdande $\mathrm{i}$ gräsens blomställningar jämte några drag ur släktet Phloeothrips utveck-lingshistoria. Entomologisk Tidskrift, 16, 157-194.

Trybom, F. (1896) Einige neue oder unvollständig beschriebene Blasenfüsse (Physapoden). Öfversigt af Kongl. Vetenskapsakademiens forhandlingar, 53 (8), 613-626

Trybom, F. (1910) Physapoda. In Schultze: Zoologische und anthropologische Ergebnisse einer Forschungsreise im westlichen und zentralen Südafrika (1903-1905). Denkschriften der Medizinisch-naturwissenschaflichen Gesellschaft zu Jena, 16, 147-174.

Trybom, F. (1911) Physapoden aus Ägypten und dem Sudan. In Jägerskiöld: Results of the Swedish Zoological Expedition to Egypt and the White Nile 1901, 19, 1-16.

Trybom, F. (1912a) Mitothrips, eine neue Physapoden-Gattung aus Britischen Ostafrika. Entomologisk Tidskrift, 33, 145-151. https://doi.org/10.5962/bhl.part.17454

Trybom, F. (1912b) Physapoden gesammelt von der Schwedischen Expedition nach dem Brittischen Ostafrika. Arkiv för Zoologi, 7 (22), 1-12.

Trybom, F. (1913) Physapoden aus Natal und dem Zululande. Arkiv för Zoologi, 7 (33), 1-52. https://doi.org/10.5962/bhl.part.17454

Tyagi, K. \& Kumar, V. (2006) A remarkable new phlaeothripid (Tubulifera: Thysanoptera) related to Haplothrips, from India. Journal of Experimental Zoology, India, 9, 337-340.

Tyagi, K., Singha, D., Saha, G.K. \& Kumar, V. (2018) One generic synonym and one new species of Phlaeothripidae from India (Thysanoptera). ZooKeys, 786, 59-68. https://doi.org/10.3897/zookeys.786.28332

Ulitzka, M.R. (2017) Revision of the fossil Melanthripidae from Baltic Tertiary (Eocene) amber including a new genus (Insecta: Thysanoptera). Entomologische Zeitschrift, 127 (4), 205-212.

Ulitzka, M.R. (2018) A first survey of Cretaceous thrips from Burmese amber including the establishment of a new family of Tubulifera (Insecta: Thysanoptera). Zootaxa, 4486 (4), $548-558$ https://doi.org/10.11646/zootaxa.4486.4.8

Ulitzka, M.R. (2021) All genera of the world: Order Lophioneurida - fossil out-groups of Thripida (Animalia: Arthropoda: Insecta). Megataxa, 6 (1), 73-75. https://doi.org/10.11646/megataxa.6.1.3

Ulitzka, M.R. \& Mound, L.A. (2014) New generic synonyms in the Palaeotropical genus Urothrips (Thysanoptera: Phlaeothripinae) with one new species from Seychelles. Zootaxa, 3755 (6), 595-600. https://doi.org/10.11646/zootaxa.3755.6.6

Uzel, H. (1895) Monographie der Ordnung Thysanoptera. Königrätz, Bohemia, 472 pp.

Varatharajan, R. \& Singh, T. (2000) A new subgenus and species of gall thrips under the genus Mesothrips Zimmerman from Nagaland. Hexapoda, 12 (1-2), 47-51.

Velikan, V.S. (1986) Two new genera and three new species of thrips (Thysanoptera) from south-eastern Kara Kum. Entomologicheskoe Obozrenie, 65 (4), 719-723. 
Vuillet, A. (1913) Description d'une nouvelle espéce et d'un nouveau genre de la famille des Phloeothripidae. Insecta, Revue Illustrée d'Entomologie, Rennes, 3 (27), 77-84.

Vuillet, A. (1914a) Une novelle espéce de Thysanoptere, de Tunisie, appartenant a un genre nouveau (Thripidae). Bulletin de la Société Entomologique de France, 6, 189-190.

Vuillet, A. (1914b) Deux Thysanoptères nouveaux du Soudan francais (Phloeothripidae). Insecta, Revue Illustrée d'Entomologie, 4 (40), 121-132.

Walker, F. (1852) List of the Homopterous insects in the British Museum Part IV. London: British Museum.

Walker, F. (1859) Characters of some apparently undescribed Ceylon insects. Annals and Magazine of Natural History, (3) $4,217-224$ https://doi.org/10.1080/00222935908697111

Wang, C.L. (2007) Hydatothrips and Neohydatothrips (Thysanoptera, Thripidae) of East and South Asia with three new species from Taiwan. Zootaxa, 1575, 47-68. https://doi.org/10.11646/zootaxa.1575.1.3

Wang, Z.-H., Mound, L.A. \& Tong, X.-L. (2019) Phylogenetic relationships within the Frankliniella genus-group based on morphology, with a revision of Iridothrips (Thysanoptera, Thripidae). Zootaxa, 4651 (1), 141-154.

https://doi.org/10.11646/zootaxa.4651.1.9

Ward, A. (1970) A new genus and species of heliothripine Thysanoptera from New Zealand. Entomologist's monthly magazine, 106, 88-90.

Watson, J.R. (1918) New Thysanoptera from Florida - IV. Florida Buggist, 2, 97-102. https://doi.org/10.2307/3491882

Watson, J.R. (1919) Additions to the Thysanoptera of Florida - V. Florida Buggist, 3, 2-7. https://doi.org/10.2307/3491845

Watson, J.R. (1920) New Thysanoptera from Florida - VII. The Florida Entomologist, 4 (2), 18-23. https://doi.org/10.2307/3492169

Watson, J.R. (1922) Another camphor thrips. The Florida Entomologist, 6 (1), 6-7. https://doi.org/10.2307/3492799

Watson, J.R. (1923) Synopsis and catalog of the Thysanoptera of North America. Bulletin of the Agricultural Experiment Station, University of Florida, 168, 1-100.

Watson, J.R. (1924) A new Bregmatothrips (Thysanoptera) from England and Holland. Entomologist's monthly Magazine, 60, 253-254.

Watson, J.R. (1926) New Thysanoptera from Florida - XIII. The Florida Entomologist, 10 (1), 9-12. https://doi.org/10.2307/3492943

Watson, J.R. (1927) New Thysanoptera from Florida XIV. The Florida Entomologist, 10 (4), 60-62. https://doi.org/10.2307/3492498

Watson, J.R. (1937a) A new Trichothrips (Haplothrips) from Alabama (Trichothrips asymmetricus n. sp.). The Florida Entomologist, 20 (1), 8-9. https://doi.org/10.2307/3492579

Watson, J.R. (1937b) Thysanoptera of the Geenton. The Florida Entomologist, 20 (1), 12-15. https://doi.org/10.2307/3492581

Watson, J.R. \& Osborn E. (1919) Additions to the Thysanoptera of Florida V. Florida Buggist, 2, 116-119. https://doi.org/10.2307/3491870

Westwood, J.O. (1838) Thysanoptera, pp 45-46 in Synopsis of the genera of British Insects 158pp in Westwood, J.O. (1840) An introduction to the modern classification of insects. $587 \mathrm{pp}$. Spottiswoode, London.

Westwood, J.O. (1880) The Pea Thrips. Gardener's Chronicle, 2, 207. London.

Williams, C.B. (1913) On two new species of Thysanoptera from the West Indies. Journal of Economic Entomology, 8, 209215.

Williams, C.B. (1914) Kakothrips n.gen., a division of the genus Frankliniella (Thysanoptera). The Entomologist, 47, 247248.

Williams, C.B. (1916) Thrips oryzae sp.nov., injurious to rice in India. Bulletin of Entomological Research, 6, 353-355. https://doi.org/10.1017/S0007485300043625

Wilson, T.H. (1972) Apollothrips bhattii, a new genus and species of thrips (Thysanoptera: Thripidae) from Central India, with a synopsis of related genera. Annals of the Entomological Society of America, 65, 49-54.

https://doi.org/10.1093/aesa/65.1.49

Wilson, T.H. (1975) A monograph of the subfamily Panchaetothripinae (Thysanoptera: Thripidae). Memoirs of the American Entomological Institute, 23, 1-354.

Yakhontov, V.V. (1956) A new genus and species of thrips from Zailisk Ala-Tai. [in Russian] Zoologicheskii Zhurnal, 35, 554-555.

Yakhontov, W.W. (1958) A new subgenus and species of the genus Dendrothrips (Thysanoptera, Thripidae). Revue d'Entomologie de l'URSS, 37 (3), 705-707.

Zetterstedt, J.W. (1828) Fauna Insectorum Lapponica Hammone, Libraria Schultziana.

Zhang, H.-R., Mound, L.A. \& Xie, Y.-H. (2010) A new genus and species from southwestern China in the Frankliniella genusgroup (Thysanoptera: Thripidae). Zootaxa, 2729, 65-68. https://doi.org/10.11646/zootaxa.2729.1.6

Zhang, S.-M., Wang, Z.-H., Li, Y.-J. \& Mound, L.A. (2018) One new species, two generic synonyms and eight new records of Thripidae from China (Thysanoptera). Zootaxa, 4418 (4), 370-378. https://doi.org/10.11646/zootaxa.4418.4.3

Zhang, W.-Q. \& Tong, X.-L. (1992) A new genus of Thripidae (Thysanoptera), with two new species from China. Entomotaxonomia, 14(2), 81-86.

Zimmermann, A. (1900) Ueber einige javanische Thysanoptera. Bulletin de l'Institut botanique de Buitenzorg, 7, 6-19.

zur Strassen, R. (1959) Studies in African Thysanoptera, 2. Journal of the entomological Society of southern Africa, 22, 174198.

zur Strassen, R. (1961) Chirothrips madagassus n.sp., die erste auf Madagascar gefundene Art dieser Gattung (Ins., Thysanoptera, Thripidae). Senckenbergiana biologica, 42, 93-97.

zur Strassen, R. (1965) Einige neue terebrante ThysanopterenArten von den Kanarischen Inseln (Ins., Thysanoptera). Commentationes biologicae Societas Scientiarum Fennica, 28 (6), 3-41.

zur Strassen, R. (1966a) Taxionomisch-systematische Bemerkungen zur Gattung Apterygothrips Priesner (Ins., Thysanoptera, Phlaeothripidae). Senckenbergiana biologica, 47 (3), 161175. 
zur Strassen, R. (1966b) Craspedothrips, eine neue aethiopische Thripiden-Gattung (Ins., Thysanoptera). Senckenbergiana biologica, 47 (6), 443-446.

zur Strassen, R. (1968a) Ökologische und zoogeographische Studien über die Fransenflügler-Fauna (Ins., Thysanoptera) des südlichen Marokko. Abhandlungen der senckenbergischen naturforschenden Gesellschaft, 515, 1-125.

zur Strassen, R. (1968b) Tabellarische Arten-Übersicht der Fransenflügler-Gattung Dendrothrips Uzel 1895 (Thysanoptera: Thripidae). Journal of the entomological Society of southern Africa, 31, 213-220.

zur Strassen, R. (1968c) New records of South African Thysanoptera with description of a new phlaeothripid genus. Journal of the entomological Society of southern Africa, 31, 365-372.

zur Strassen, R. (1969) Zwei neue Chirothrips-Arten aus Südafrika (Ins., Thysanoptera, Thripidae). Senckenbergiana biologica, 50, 171-177.

zur Strassen, R. (1972) Äthiopische Thysanopteren überwiegend aus dem Massiv des Mount Elgon in Kenya (Insecta, Thysanoptera). Zoologica Scripta, 1, 85-105. https://doi.org/10.1111/j.1463-6409.1972.tb00572.x

zur Strassen, R. (1973) Fossile Fransenflügler aus mesozoischem Bernstein des Libanon (Insecta: Thysanoptera). Stuttgarter Beiträge zur Naturkunde (A), 256, 1-51.

zur Strassen, R. (1974) Neue silvicole Fransenflügler von den makronesischen Inseln (Ins.: Thysanoptera). Senckenbergiana biologica, 55, 105-134.

zur Strassen, R. (1976) Thysanoptera. In Basilewski, P. \& Decelle, J. [eds] La faune terrestre de l'île de Sainte-Hélène. Annales de la Musée royal de l'Afrique central, 215, 236-256.

zur Strassen, R. (1977) Studie zur Frühjahrsfauna der Fransenflügler auf den Madeira-Inseln im Atlantik nebst Daten zur Abundanz und Faunistik (Insecta: Thysanoptera). Boletim do Museu municipal Funchal, 31, 5-78.

zur Strassen, R. (1979) Insects of Saudi Arabia. Thysanoptera. In Wittmer, W. \& Buttiker, W. [eds] Fauna of Saudi Arabia, 1, 90-104.

zur Strassen, R. (1980a) Thysanopterologische Notizen (5) (Insecta: Thysanoptera). Senckenbergiana biologica, 60, 191-202.

zur Strassen, R. (1980b) Anactinothrips gibbifer n.sp. aus Baumkronen im Amazonas-Gebiet (Insecta: Thysanoptera: Phlaeothripidae). Senckenbergiana biologica, 61, 47-56.

zur Strassen, R. (1989) The biogeographical character of the Thysanoptera fauna (Insecta) of Andalusia, Spain. Acta phytopathalogica et entomologica Hungarica, 23 [1988], 351-359.

zur Strassen, R. (1993) Chorologische, phänologische und taxonomische Studien an Terebrantia der Kapverden (Insecta: Thysanoptera). Courier Forschungsinstitut Senckenberg, 159, 335-380.

zur Strassen, R. (1995) Taxonomische Neuerungen bei terebranten Fransenflüglern der westlichen Paläarktis (Thysanoptera: Terebrantia: Aeolothripidae, Thripidae). Mitteilungen des internationalen entomologischen Vereins, 20, 87-102.

zur Strassen, R. (1997) Blascothrips zumetai n.gen. n. sp., ein autumnaler steppenbewohnender Fransenflügler aus dem nördlichen Spanien (Thysanoptera: Thripidae). Entomologische Zeitschrift, 107, 142-148.

zur Strassen, R. (2000) Thysanopterologische Notizen (7) (Thysanoptera: Terebrantia). Entomologische Nachrichten und Berichte, 44, 25-34.

zur Strassen, R. (2003) Die terebranten Thysanopteren Europas und des Mittelmeer-Gebietes. Die Tierwelt Deutschlands, 74, $1-271$. 